\title{
A MEASUREMENT OF THE NEUTRAL CURRENT NEUTRINO-NUCLEON ELASTIC CROSS SECTION AT MINIBOONE
}

\section{David Christopher Cox}

Submitted to the faculty of the University Graduate School in partial fulfillment of the requirements

for the degree

Doctor of Philosophy

in the Department of Physics,

Indiana University

February 2008 
Accepted by the Graduate Faculty, Indiana University, in partial fulfillment of the requirements for the degree of Doctor of Philosophy.

Doctoral Committee

Rex Tayloe, Ph.D.

Charles J. Horowitz, Ph.D.

Robert R. de Ruyter, Ph.D.

Jon Urheim, Ph.D.

January 10, 2008 
to my parents 


\section{Acknowledgements}

I used to wonder why scientists go mad. These are people whose expertise is observing the universe from a logical, rational, objective viewpoint. What traumatic experience could possibly instigate their exodus into Banana Nut Sundae Land? What could break their psyche to the point where they would plot to take over the world with armies of flying kewpie dolls, or bring grape jelly to life with old car batteries? Was it the radiation, the fumes from the neighboring chemistry lab? I thought I would never know the answer.

Then I went to grad school.

And now I wonder why every scientist isn't mad - though I have a sneaking suspicion that most actually are. And since I have just finished the journey myself, I would like to thank the many people who have encouraged and supported (and occasionally pushed) me into this land covered with whipped cream and topped with a maraschino cherry.

To all my fellow MiniBooNE collaborators: It's been great to be a part of this experiment and to work alongside you. I am grateful for your expertise, insight, support, and encouragement. To those of you with whom I have had the good fortune to work closely, I offer individual thanks.

To my advisor, Rex Tayloe: Congratulations! This is your victory as much as mine. It took more time and bootstrapping than we originally expected, but we managed to produce MiniBooNE's first differential cross section, and I'd say we've told the story well. I'm glad 
I had you to guide me past all the devils in the details. And I hope I've been able to pick up some of that knack you have for knowing the best place to attack a problem, your dogged enthusiasm to find the solution, and your insistence on doing things right. You are an excellent physicist, and I'm proud to have had you as my advisor.

To Chris Polly, my postdoc (that's right, my postdoc, though I guess I can share): You've been a great academic uncle - the devil on my shoulder, encouraging me to do the things I really want to but know I shouldn't (like getting that next beer at lunch...and then the one after that...). Thanks for being so enthusiastic about all the projects you were working on, and yet so interested in understanding everyone else's. I look forward to following your example and telling everyone at my new job, "When I was a grad student on MiniBooNE, we...".

To Sam Zeller: What up? Talking to you was guaranteed to brighten my day. You always made me feel that my research was meaningful and maybe even a little excitingpriceless gifts for a grad student. Morgan Wascko: Have you ever wondered how Leon Phelps would sound as the voice of Darth Vader? "Don't unduh-eth-timate the pow-uh of the Fothe,"-it adds new meaning. Thanks for making this all seem like fun. Yong Liu, thank you for explaining and re-explaining with endless patience and elegant diagrams what exactly goes on inside the Stancu Fitters (though I'm still not sure I totally understand). Gordon McGregor, I couldn't have asked for anyone better to defend the BooNE House with me against the aggressive Tevatron N.I.M.H. rats. Hiro Tanaka thanks for teaching me that you have to get up early to catch neutrinos, no matter what the activities of the previous night. Jon Link, thank you for naming the first neutral current elastic selection set after me-although "Cox Cuts" still makes me a little nervous for some reason.

Hello, Jen Raaf. I owe you special thanks for combing Dorm 3 with Shawn to find me on my first evening at the lab. Thank you for teaching me how to make the Fermilab water palatable, impressing on me the importance of second hand smoke breaks, and showing me 
the awesome power of ManBat. I'm not sure I would have made it without your guidance and friendship. Jocelyn Monroe, always professional and chic. Thank you for warmly welcoming me into your office when I was kicked out of mine. Michel Sorel: Ciao! Everything had a more Mediterranean atmosphere when you were around. Dave Schmitz, I'm not sure whether to thank you or hate you for taking me sailing because now I will not be satisfied until I have a boat of my own. Thank you for always providing a balanced perspective.

To Teppei Katori: Cześć, Stary! I'll never forget the day you arrived at the Cyclotron with a suitcase full of physics books, singing Elvis, and playing the blues on your miniharmonica. Alright, so maybe that's a little exaggerated...you probably had some clothes in that suitcase as well. It has been an honor to work with you, to watch you mature into the formidable researcher you are now. Your untiring dedication has always inspired me to work harder and be a better person. I have enjoyed hanging out with you and watching your many exploits: you are definitely a bad ass. You have been a great friend, and I wish you the best of success and happiness as you continue in your career.

Bob Nelson, you are a dangerous man. My productivity always fell drastically when we were at the lab together...and I am very grateful for it. Thanks for helping to keep me focused on the things that are really important. Serge Ouedraogo, thank you for being excellent company when I was at the lab. I enjoyed our sympathetic conversations about research and also learning about Burkinabé culture. Kendall Mahn, thank you for putting into words exactly what I've felt it's been like to be a grad student. You naturally do what it takes to be successful, and I think you're going to really shine. Alexis Aguilar-Arevalo, you have the perfect name for this sort of work. Thank you for being so positive and so open. Denis Perevalov, a.k.a. Денис Перевалов, your work is already an improvement over mine, and I'm looking forward to seeing the final results. Thank you for your enthusiasm for this research.

To the fearless spokespersons of MiniBooNE, Janet Conrad, Bill Louis, Steve Brice, 
and Richard Van der Water: Thank you for your leadership, optimism, and support. I am grateful to each of you for benefiting my research directly via insight you shared, expertise you offered, or analysis you performed. (P.S. Richard, I guess I've finally eaten enough chicken wings. If I had known it would have taken this much I would have just bought a chicken farm.)

As well as my MiniBooNE colleagues, I have also been fortunate to work with outstanding people in the IUCF neutrino group. To Nate Walbridge: Thanks for taking me out before my first MiniBooNE meeting so that I was sure I could feel the High Rise sway in the breeze the next morning. And thank you for following exactly the path you set out for yourself. Patrick Ockerse, thank you for demonstrating that Pepsi is worse for millipedes than all strengths of La Bamba's salsa. Thanks for your "stadium laugh", your ability to somehow do all your work and have time to play, and for your always positive attitude. To Sara "The Green Diamond" Walbridge: Thanks for being excellent company throughout my time at IU, for shaking the sample like a pro, and for sharing your understated humor and superb insight. I can't tell you enough how glad I am that you came back. Shawn McKenney, thanks for being the expert despite a degree, for teaching me about homemade smoked apples, and for dispensing wisdon with a Yooper accent. To Melanie Boy nèe Novak: I am grateful to you for adding much needed color and frangrance to our office, it was sorely missed when you left. Thanks for sharing your gigantic box of Blo Pops (I think there are still some left). And Jonathon Horton, it was a pleasure to end my time at IU in your company: I really appreciated your unique sense of humor and hope things work out for you.

I would like to offer my sincere gratitude to the members of my dissertation committee, Chuck Horowitz, Rob de Ruyter, and Jon Urheim, for agreeing to review my work. I appreciate your time and effort in this as well as all of your comments and questions.

To the Physics Faculty of Indiana University, I would like to extend my thanks for your 
guidance along this path to my doctorate. Go IU! In particular, I would like to thank Hans Meyer for providing vital insight into the techniques and the attitude necessary to be a good experimentalist and for having so much fun in the process. Malcolm McFarlane, thank you for taking a summer to help my class study for our Qualifying Exam. I believe I owe much of my passing grade to your tutelage and insight. Brian Serot, I have never had to solve more intense problems than for your Field Theory class. Thanks for your encouragement, and for engaging conversations about cigars and booze.

In any department, the people who really make things happen are the secretaries. I consider myself lucky to have worked with the best at the University. In the Physics Department, I would like to thank Bev Carson, June Dizer, Tracey McGookey, and Debbie McKinney for making sure I was always signed up for classes, had a valid contract, and otherwise on top of my paperwork. At the IUCF, thanks to Carol Glaze and Marcia Strain for ensuring that I always made it to my final destination when I travelled (and that I got a speedy reimbursement when I returned). Thanks also to Amy Hoover, Janet Meadows, Moya Wright, Jane Decker and Maggie Ochoada for keeping me out of trouble and making life at the Cyclotron generally nicer.

The ascent into madness is always more fun in the company of friends, and I've been fortunate to have good friends. I would like to thank the Polish Mafia: Pawel \& Ewa, Crystal, Jorge, Łukas, Carrie, Kurt, and Dan, for getting me through those initial years as a grad student. Despite the arduous course load, my memories of that time are positive because of you. To the IU Shotokan Karate Club, thank you for teaching me how to move and how to be still, but mainly for giving me a safe place to hit things. To the Karaoke Mafia: George, Jay, Brandon, Jason, Heechang, and company, thank you for for letting me vicariously relive my first-year days through you. It was almost as much fun the second time. Liliana Cabellero, I've enjoyed all the time I've been able to spend with you. Your infectious smile has brightened more days for me than I can remember. Lenilson Coutinho, 
I wish I could be as relaxed about life as you. I loved talking to you and learning about Brazil. Dan Duggan, thank you for always having time for me when I was at Fermilabparticularly when you visited me on shift. Leah Welty and Jason Rieger, it was great to hang out with you guys and compare notes on our experiences. I wish you both the best. To Brad B., thank you for being my personal brain doctor; I am more myself because of our time together. Margarita Valentin: thanks for being direct, fearless, and loud (in a word, Latin), and for teaching me where the power in a hospital really lies. Tahriah AkbarWilliams thank you for being able to be the star of the show wherever you are. Heather Davis, thanks for not giving up on me despite my lack of communication.

To Rafael \& Jamie Valentin and Lisa Pierson: It's my day! You guys have been my family here in Bloomington-I can tell because you pick on me as much as they do. Rafael, you're a modern Renaissance Man: a martial artist painter with a motorcycle that owns a spectrometer and can converse intelligently about warp nacelles. We've talked about risk and reward, but I most value learning that nothing is really worthwhile unless you do it 100\%: body, mind, and soul. Jamie, with more letters after your name than in it, I love having conversations with you that jump between hypotheses, topics, and themes, yet staying completely in the rhythm of each other's thoughts. Thank you for perky mornings, tostones, and tequila night. Lisa, a.k.a. Tater Salad, thank you for calling me out on everything, for horsing around with me like we're kids, and then taking me seriously when it matters, for getting me a stethoscope when I became a doctor, and for naming me Trisha.

Nikodem Popławski. You have been the best friend anyone could ask for-better than anything I could have ever imagined, and definitely better than I deserve. If there is really such a thing as karma, you must have lived a really terrible life. I know I would not be here without you: you studied with me every day the summer before my qualifier, and since then have gladly helped me with my most naive math or physics questions. You are always supportive, trying to come up with solutions to my problems, or offering to smite 
my enemies. You have a very unusual sense of humor (some strange amalgamation of Mr. Bean, Miś, and 'Allo 'Allo!) that always makes me smile. Thank you for knowing the significance of every day (like March 42nd is the day Einstein met Pavarotti), for paying attention to strange details and finding them fascinating, for celebrating every little victory and occasion. Thank you for your generosity and gentleness: for never holding a grudge and for always taking time to be there when I really need you. Thank you for sharing your family with me: it's clear where you get your warmth and openness. Thank you for everything; I hope you'll always be part of my life.

At the beginning of this I wrote that grad school made me lose my mind, yet looking back on my life, I'd say that my family gave me a good head start - a double whammy of nature and nurture. But, Family, despite both being crazy yourselves and making me crazy, you all have never stopped encouraging me, supporting me and challenging me. And although you say you'd be proud of me no matter what, I'm glad to have something to show for all your hard work and to be able to share this success with you. So to my greatgrandfather Harold Cox and his sister Evelyn Taylor, my grandparents David \& Nancy Cox and Frances (FAB) \& Jacque Borelli, to my brother Matt and sister-in-law Beth and their two wonderful children Reilly and Gabriel, and to my parents Dave and Rose: thank you all for your support - educational, financial, emotional, and culinary (because there's nothing better for the soul than home cooked food).

Mom and Dad, you deserve individual recognition. My decision to go into the physical sciences was heavily influenced by you: all those science fiction movies, trips to planetariums, dressing me up as a mad scientist for Halloween, and advising me to take an employable career. What I'm trying to say is that I would never have gotten here without you. Or to put it more succinctly: This is all your fault. Thank you for being a constant source of encouragement, emotional support, big picture perspective, and good advice throughout the whole process. And thanks for starting me down this path. Who knew that it was the 
kind of career where being nuts is not a handicap for employment, in fact it's probably an asset. In any case, it's not just a Halloween costume any more.

And now to return to my collection of kewpie dolls and old car batteries. 
David Christopher Cox

\section{A MEASUREMENT OF THE NEUTRAL CURRENT NEUTRINO-NUCLEON ELASTIC CROSS SECTION AT MINIBOONE}

The neutral current neutrino-nucleon elastic interaction $\nu N \rightarrow \nu N$ is a fundamental process of the weak interaction ideally suited for characterizing the structure of the nucleon neutral weak current. This process comprises $\sim 18 \%$ of neutrino events in the neutrino oscillation experiment, MiniBooNE, ranking it as the experiment's third largest process. Using $\sim 10 \%$ of MiniBooNE's available neutrino data, a sample of these events were identified and analyzed to determine the differential cross section as a function of the momentum transfer of the interaction, $Q^{2}$. This is the first measurement of a differential cross section with MiniBooNE data. From this analysis, a value for the nucleon axial mass $M_{A}$ was extracted to be $1.34 \pm 0.25 \mathrm{GeV}$ consistent with previous measurements. The integrated cross section for the $Q^{2}$ range $0.189 \rightarrow 1.13 \mathrm{GeV}^{2}$ was calculated to be $(8.8 \pm 0.6($ stat $) \pm$ $0.2($ syst $)) \times 10^{-40} \mathrm{~cm}^{2}$. 


\section{Contents}

Acknowledgements $\quad$ iv

\begin{tabular}{lll}
\hline 1 & Neutrinos & 1
\end{tabular}

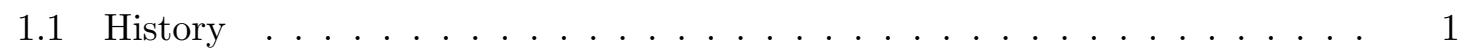

1.2 Neutrinos in the Standard Model and Neutrino Mass . . . . . . . . . . . . . 3

1.3 Neutrino Oscillation Formalism . . . . . . . . . . . . . . . 6

1.4 Oscillation Landscape $\ldots \ldots \ldots$. . . . . . . . . . . . . . . . 9

1.5 MiniBoonE Results $\ldots \ldots \ldots \ldots \ldots \ldots$. . . . . . . . . . . . . . . . . . .

1.6 Synopsis $\ldots \ldots \ldots \ldots \ldots \ldots$. . . . . . . . . . . . . . . . . . . . . . . . . 14

2 Neutral Current Elastic Cross Sections

2.1 History $\ldots \ldots \ldots \ldots \ldots \ldots$

2.2 Nucleon Neutral Weak Current . . . . . . . . . . . . . . . . . . 17

$2.3 \quad$ Measuring Nucleon Structure with Electrons $\ldots \ldots$. . . . . . . . . . . . . . 19

2.4 Neutrino-Nucleon Elastic Scattering . . . . . . . . . . . . . . . . . . . 21

$2.5 \quad$ Measuring Nucleon Structure with Neutrinos . . . . . . . . . . . . . . . . . 24

2.6 Neutral Current Elastics in MiniBoonE $\ldots \ldots \ldots \ldots \ldots$ 


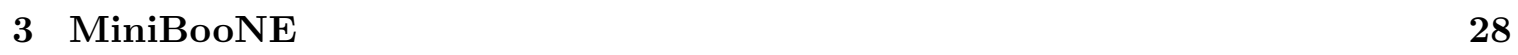

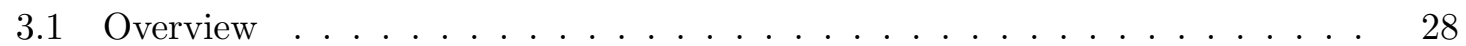

3.2 Beam . . . . . . . . . . . . . . . . . . . . . . . . . . 29

$3.2 .1 \quad$ Primary (Proton) Beam . . . . . . . . . . . . . . . . . . 30

3.2 .2 Secondary (Meson) Beam . . . . . . . . . . . . . . . . . . 31

3.2 .3 Tertiary (Neutrino) Beam . . . . . . . . . . . . . . . . 33

3.3 Detector $\ldots \ldots \ldots \ldots \ldots \ldots$

3.4 Data Acquisition . . . . . . . . . . . . . . . . . . . . . . 40

3.5 Calibration Systems $\ldots \ldots \ldots \ldots$. . . . . . . . . . . . . . . . 42

3.5.1 PMT Response \& Calibration . . . . . . . . . . . . . . . . . . . . . 42

3.5 .2 Detector Calibration . . . . . . . . . . . . . . . 46

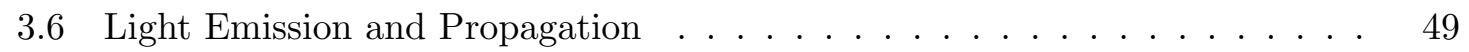

3.6 .1 Light Emission $\ldots \ldots \ldots \ldots$

3.6 .2 Light Transmission . . . . . . . . . . . . . . . . . . . . 52

4 Event Simulation and Reconstruction $\quad 54$

4.1 Beam and Flux Simulations . . . . . . . . . . . . . . . . . . . . 54

$4.2 \quad$ Neutrino Interactions Simulation . . . . . . . . . . . . . . . . . . . 59

$4.2 .1 \quad$ Quasi-Elastic Scattering . . . . . . . . . . . . . . . . . . . 59

4.2 .2 Single pion production $\ldots \ldots \ldots$. . . . . . . . . . . 62

$4.2 .3 \quad$ Final State Interactions $\ldots \ldots \ldots$. . . . . . . . . . . . . . 63

4.3 Detector Simulation $\ldots \ldots \ldots$. . . . . . . . . . . . . . . . . . . . . 64 
4.4 Simulation Uncertainties . . . . . . . . . . . . . . . . . . . . . . . . 67

4.4 .1 Beam and Flux Uncertainties . . . . . . . . . . . . . . . . 67

4.4 .2 Neutrino Interaction Uncertainties $\ldots \ldots \ldots \ldots \ldots$

4.4 .3 Detector Simulation Uncertainties $\ldots \ldots \ldots \ldots$

$4.4 .4 \quad$ Dirt Neutrino Model Uncertainties . . . . . . . . . . . . . . . . 70

4.5 Event Reconstruction: Subevents . . . . . . . . . . . . . . . . . 71

$4.6 \quad$ Event Reconstruction: Fitting Events _. . . . . . . . . . . . . . . . 72

4.6 .1 Stancu Fast Fitter $\ldots \ldots \ldots \ldots \ldots$

$4.6 .2 \quad$ Stancu Full Fitter $\ldots \ldots \ldots \ldots$

4.6 .3 Stancu Flux Fitter . . . . . . . . . . . . . . . . . . . . . . 73

$\begin{array}{lll}5 \text { Data Selection } & 75\end{array}$

$5.1 \quad$ Data Quality . . . . . . . . . . . . . . . . . . . . 75

5.2 Neutral Current Pre-Selection . . . . . . . . . . . . . . . . . . . 76

5.3 Proton PID \& Beam Unrelated Backgrounds $\ldots \ldots \ldots \ldots \ldots \ldots$

$5.3 .1 \quad$ Proton Particle Identification $\ldots \ldots \ldots \ldots \ldots$

$5.3 .2 \quad$ Normalizing The Unrelated Background $\ldots \ldots \ldots \ldots$. . . . . . 81

5.4 "Dirt" Backgrounds $\ldots \ldots \ldots \ldots \ldots \ldots \ldots \ldots \ldots$

$5.5 \quad$ Cuts Summary $\ldots \ldots \ldots \ldots \ldots$

$5.6 \quad$ Neutrino Backgrounds $\ldots \ldots \ldots \ldots \ldots \ldots$ 
$\begin{array}{llr}6 & \text { MiniBooNE NCE Results } & 89\end{array}$

$6.1 \quad$ Energy calibration $\ldots \ldots \ldots \ldots \ldots$

$6.2 \quad$ Error Propagation $\ldots \ldots \ldots \ldots \ldots \ldots$

6.2 .1 Multisims . . . . . . . . . . . . . . . . . . . . . . 95

6.2 .2 Unisims $\ldots \ldots \ldots \ldots \ldots \ldots$

6.2 .3 Error Matrices $\ldots \ldots \ldots \ldots$

$6.3 \quad$ Determining $M_{A}$ from Nucleon Kinetic Energy $\ldots \ldots \ldots$

6.4 Unfolding The Differential Cross Section . . . . . . . . . . . . . . . . . . 102

6.5 The Neutral Current Elastic Cross Section . . . . . . . . . . . . . . . . . 106

6.6 Summary and Conclusions . . . . . . . . . . . . . . . . . . . . . . . . 108

\begin{tabular}{|lr}
\hline A Slow Monitoring & 112
\end{tabular}

A.1 Measured Quantities . . . . . . . . . . . . . . . . . . . . . . . 112

A.1.1 Temperatures . . . . . . . . . . . . . . . . . . 114

A.1.2 Levels . . . . . . . . . . . . . . . . . . . . . . . . . . . . . . 114

A.1.3 Pressures . . . . . . . . . . . . . . . . . . . . 115

A.1.4 Oil Flow Rate. . . . . . . . . . . . . . . . . . . . . . . . 115

A.1.5 Relative Humidities . . . . . . . . . . . . . . . . . . . 115

A.1.6 Oxygen Levels $\ldots \ldots \ldots \ldots$

A.2 Nitrogen System . . . . . . . . . . . . . . . . . . . . 116

A.2.1 Nitrogen Bubbling . . . . . . . . . . . . . . . . . . 120

A.2.2 Nitrogen Blanket . . . . . . . . . . . . . . . . . . . . 120 
A.2.3 Level Monitoring . . . . . . . . . . . . . . . . . . . . . . . . . . . . 121

A.2.4 $\quad$ Pre Oil Fill . . . . . . . . . . . . . . . . . . . . . . . . . . . . . . . . . . 121

A.3 Remote Monitoring System . . . . . . . . . . . . . . . . . . . . . . . . 121

A.4 Summary . . . . . . . . . . . . . . . . . . . . . . . 122 


\section{List of Tables}

$3.1 \quad$ Selected photomultiplier tube properties. . . . . . . . . . . . . . . 36

$3.2 \quad$ Selected measurements of oil properties. . . . . . . . . . . . . . . 38

3.3 Locations of laser flasks $\ldots \ldots \ldots$. . . . . . . . . . . . . . . 43

5.1 Cherenkov threshold for particles in mineral oil. . . . . . . . . . . . . 78

5.2 Neutral current elastic sample selection. . . . . . . . . . . . . . 86

$5.3 \quad$ Effect of data selections on NCE purity and efficiency. . . . . . . . . . . 86

A.1 Summary of measured quantities. . . . . . . . . . . . . . . . . . 117

A.2 Slow Monitoring abbreviations. . . . . . . . . . . . . . . . . . . 118

A.3 Voltage to physical unit conversion factors for webDAQ data. . . . . . . . . 123

xviii 


\section{List of Figures}

1.1 The particles of the Standard Model of particle physics. . . . . . . . . . . . 3

1.2 Depiction of NC and CC neutrino vertices. . . . . . . . . . . . . 4

$1.3 \quad$ Measured oscillation regions in $\Delta m^{2}$ vs $\sin ^{2} 2 \theta$ space. . . . . . . . . . 10

1.4 Measured 90\% confidence limit for MiniBooNE analyses. . . . . . . . . . . . 13

2.1 Neutral current nucleon vertex. . . . . . . . . . . . . . . . . . . . 17

$2.2 \quad$ PVES electron scattering amplitudes. . . . . . . . . . . . . . . . 20

$2.3 \quad$ Schematic figure of a DIS process. . . . . . . . . . . . . . 20

2.4 Diagrams for NCE and CCQE scattering. . . . . . . . . . . . . . 22

$2.5 \quad$ BNL E734 differential cross section results. . . . . . . . . . . . . . . . 25

3.1 MiniBooNE beamline and detector cartoon . . . . . . . . . . . . . . 29

3.2 Schematic view of the $8 \mathrm{GeV}$ Fixed Target Facility . . . . . . . . . . . 30

$3.3 \quad$ Rendering of the horn with transparent outer conductor. . . . . . . . . . . 32

3.4 Rendering of the horn and cooling water system. . . . . . . . . . . . . 33

3.5 Cut-away of the MiniBoonE detector. . . . . . . . . . . . . . 35

3.6 MiniBooNE PMT in wire support-frame. . . . . . . . . . . . . . . . 36 
$3.7 \quad$ Photograph of main detector and veto regions. . . . . . . . . . . . . . . 37

$3.8 \quad$ Elevation drawing of the MiniBooNE Detector Plant. . . . . . . . . . . . . . . 39

$3.9 \quad$ Perspective drawing of the MiniBooNE Support Plant. . . . . . . . . . . . . . 39

3.10 DAQ charge and time traces. . . . . . . . . . . . . . . . 41

3.11 General PMT internal structure. . . . . . . . . . . . . . . . . . . 44

3.12 Schematic of photoelectron paths to the first dynode. . . . . . . . . . . . 44

3.13 Quantum efficiencies for old and new tubes. . . . . . . . . . . . . . 45

3.14 Calibrated time distribution of R1408 PMTs $\ldots \ldots \ldots$. . . . . . . 47

3.15 Cosmic muon energy calibration. . . . . . . . . . . . . . . 48

3.16 Observed Michel electron energy distribution. . . . . . . . . . . . . . . . 49

3.17 Cherenkov radiation schematic. . . . . . . . . . . . . . 51

3.18 Attenuation rate in MiniBooNE mineral oil vs wavelength. . . . . . . . . . 52

$4.1 \quad$ HARP $\pi^{+}$data with Sanford-Wang fits . . . . . . . . . . . . . 56

$4.2 \quad p_{t}$ vs $x_{F}$ phase space for MiniBooNE $K^{+}$production $\ldots \ldots \ldots \ldots$

$4.3 \quad$ Predicted $\nu_{\mu}$ and $\nu_{e}$ fluxes as a function of neutrino energy. . . . . . . 58

$4.4 \quad$ Neutral current elastic proton and neutron Nuance cross sections. . . . . . . 61

4.5 Detector Monte Carlo detector geometry including dirt. . . . . . . . . . . . 65

5.1 Monte Carlo neutral current elastic THits distribution. . . . . . . . . . . . . 77

$5.2 \quad$ Prompt hits fraction as NCE particle ID $\ldots \ldots \ldots \ldots \ldots$

$5.3 \quad$ Prompt hits fraction for CCQE and NCE events. . . . . . . . . . . . . . . 81

$5.4 \quad$ Normalizing beam-unrelated backgrounds before PID. . . . . . . . . . . . 82 
$5.5 \quad$ Normalizing beam-unrelated backgrounds after PID. . . . . . . . . . . . . 83

5.6 Reconstructed volume for events generated inside and outside the detector. $\quad 84$

5.7 Composition of NCE data sample after all cuts. . . . . . . . . . . . . . 87

$\begin{array}{lll}5.8 & \text { Predicted cross section for irredcible backgrounds. } \ldots \ldots \ldots & \ldots\end{array} \ldots$. . . . 88

$6.1 \quad$ Nucleon kinetic energy calibration for Monte Carlo events. . . . . . . . . . . 90

6.2 Light flux kinetic energy versus caibrated kinetic energy. . . . . . . . . . . . 91

6.3 Cherenkov threshold transition for NCE events. . . . . . . . . . . . . . . . . 91

6.4 Calibrated KE for NCE data and strobe background. . . . . . . . . . . . . . 92

6.5 Calibrated KE for Monte Carlo backgrounds. . . . . . . . . . . . . . . . . . 93

6.6 Calibrated KE for NCE data and simulations with errors. . . . . . . . . . . 94

6.7 Beam/flux and cross section systematic errors. . . . . . . . . . . . . 96

6.8 Light propagation and other detector model errors. . . . . . . . . . . . . . . 97

$6.9 \quad$ Dirt model and kinetic energy calibration errors. . . . . . . . . . . . . . . 97

6.10 The complete relative error matrix. . . . . . . . . . . . . . . . . . . . 99

$6.11 \Delta \mathrm{s} M_{A} \chi^{2}$ parameter space. . . . . . . . . . . . . . . 100

6.12 Calibrated $T_{N}$ with different values of $M_{A}^{Q E}(\Delta \mathrm{s}=0) . \ldots \ldots \ldots$

6.13 NCE efficiency matrix $\ldots \ldots \ldots \ldots$. . . . . . . . . . . . . 103

6.14 Generalized efficiency for NCE, NCp, and NCn events. . . . . . . . . . . . 105

6.15 NCE data unfolded cross section with errors. . . . . . . . . . . . . . . 107

6.16 NCE data unfolded cross section with simulated prediction. . . . . . . . . 109

6.17 NCE data unfolded cross section with BNL 734 data. . . . . . . . . . . . . . 110 
A.1 Environmental probe locations in the detector, and local display. . . . . . . 113

A.2 Schematic of the $N_{2}$ flow system. $\ldots \ldots \ldots \ldots \ldots \ldots$

A.3 Remote slow monitoring data. . . . . . . . . . . . . . . . . . 124 


\section{Chapter 1}

\section{Neutrinos}

Neutrinos are very light, electrically neutral particles with intrinsic angular momentum (spin) $\hbar / 2$ that interact only by means of the weak nuclear force via the $W^{ \pm}$and $Z^{0}$ bosons. They exist in three "flavors" $\nu_{e}, \nu_{\mu}$, and $\nu_{\tau}$ corresponding to the three flavors of charged leptons. Sources of neutrinos include the Big Bang (called relic neutrinos), supernovae and other energetic cosmic events, the Sun, the atmosphere, the earth, and man-made sources such as nuclear reactors and accelerators. They provide a means to study environments that could not otherwise be observed (such as the center of the sun), as well as a tool for probing the boundaries of the Standard Model of particle physics. A brief overview of neutrinos is presented in this chapter.

\subsection{History}

In 1930, the scientific community was torn by the apparent non-conservation of energy and momentum in nuclear beta decay. Experiments by Lise Meitner, Otto Hahn and others had demonstrated that the electrons emitted in these decays followed a continuous energy spectrum and not the discrete one predicted for two-body decays. Although some physicists 
(notably Niels Bohr) suggested abandoning energy conservation, Wolfgang Pauli proposed the existance of an additional particle produced in beta decays that was light and neutral to carry some of the energy and preserve conservation [1]. Enrico Fermi, who developed the first theory of weak interactions, named these particles "neutrinos."

In 1956, Frederick Reines and Clyde Cowan saw the first experimental signature of neutrinos from a nuclear reactor using "inverse" beta decay: $\bar{\nu} p \rightarrow n e^{+}[2,3]$. A few years later in 1962, Lederman, Schwartz, and Steinberger showed that the neutrino resulting from the decay of charged pions (the muon neutrino) was not the same flavor as the one observed by Reines and Cowan (the electron neutrino) [4]. The tau neutrino was later discovered in 2000 by the DONUT collaboration at Fermilab [5] verifying the hypothesis that all three flavors of charged lepton have a corresponding neutrino.

Along with the discovery of neutrino flavors in the 1960's, other experiments were created to measure neutrino fluxes from sources such as the sun, the atmosphere, reactors, and accelerators. Some of these experiments saw a different number of neutrinos than predicted. A way to rectify the differences came from a 1957 idea by Bruno Pontecorvo that massive neutrinos can oscillate between flavor states [6, 7]. This was confirmed in 1998 by the SuperK experiment [8] after decades of mounting evidence in the solar and atmospheric neutrino sectors. However, this did not complete the neutrino oscillation story. One remaining issue was the unconfirmed oscillation result reported by the LSND experiment. This was not addressed until MiniBooNE released its oscillation results in the Spring of 2007.

To provide context for the MiniBooNE results described at the end of this chapter, an introduction to the relevant physics of neutrinos is presented next, beginning with their place in the Standard Model of particle physics. 


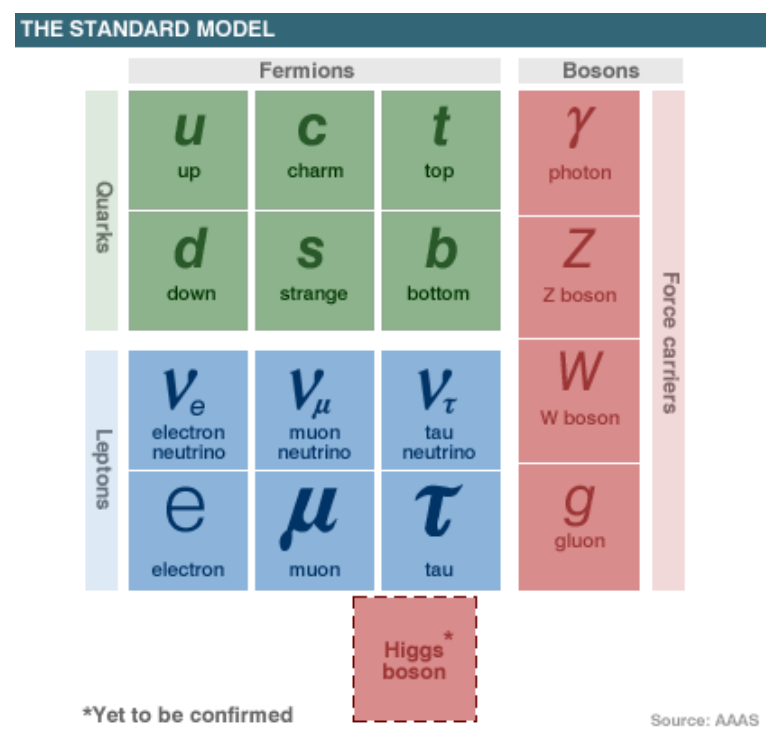

Figure 1.1: The particles of the Standard Model of particle physics. [9]

\subsection{Neutrinos in the Standard Model and Neutrino Mass}

The Standard Model describes the properties of the electromagnetic, strong nuclear, and weak nuclear interactions. The model predicts that elementary particles interact with each other via force carriers called gauge bosons (figure 1.1). The boson for electromagentism is the photon $\gamma$; the gluon $g$ mediates the strong force, and two charged bosons $W^{ \pm}$plus a neutral boson $Z^{0}$ convey the weak force. The quarks interact via all of these force carriers, and the charged leptons couple to both electromagnetism and the weak nuclear force. However, only the bosons of the weak force (the $W^{ \pm}$and $Z^{0}$ ) mediate the interactions of neutrinos.

When a neutrino exchanges a $Z^{0}$, the process is called "neutral current" (NC). When a neutrino interaction is instead mediated by one of the $W^{ \pm}$, it is called "charged current" (CC) (figure 1.2). These bosons are heavy, larger than $80 \mathrm{GeV}$ (or 80 times as massive as a proton) making them very short lived particles. The large amounts of energy required to 

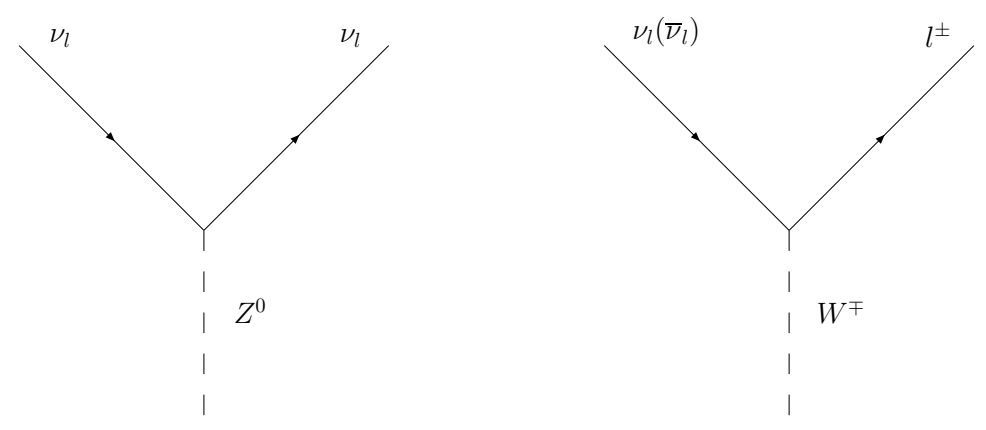

Figure 1.2: Depiction of neutral current and charged current neutrino vertices using JaxoDraw [10].

produce particles as large as the weak gauge bosons greatly reduces the probability that a weak event will occur, and thus causes the rarity of neutrino interactions.

Neutrinos, charged leptons, and quarks are all fermions: they all have intrinsic angular momentum (spin) equal to $\hbar / 2$ (or $1 / 2$ in "natural" units where $\hbar=1$ ) and obey the Pauli exclusion principle. Moreover, they are all Dirac fermions, which means that they can be projected into left- and right-handed states. Specifically, the handed (chiral) projections of a Dirac fermion $\nu$ are

$$
\nu_{L, R}=\frac{1}{2}\left(1 \mp \gamma^{5}\right) \nu, \quad \bar{\nu}_{L, R}=\frac{1}{2}\left(1 \pm \gamma^{5}\right) \bar{\nu}
$$

where $\bar{\nu}$ is the adjoint of $\nu$ and not its antiparticle $\ell^{1}, \gamma^{5}=i \gamma^{0} \gamma^{1} \gamma^{2} \gamma^{3}$, and $\gamma^{0 \rightarrow 3}$ are the Dirac matrices.

Handedness (chirality) is a Lorentz invariant property: a left-handed particle is lefthanded in all frames. This is important for weak interactions. Both the electromagnetic and strong forces preserve parity, the symmetry between left- and right-handedness. However, the weak nuclear force violates parity and so interacts differently with the two chiral states.

\footnotetext{
${ }^{1}$ Physically $\bar{\nu}_{L}\left(\bar{\nu}_{R}\right)$ creates a LH $(\mathrm{RH})$ neutrino and destroys a RH (LH) antineutrino, and $\nu_{L}\left(\nu_{R}\right)$ destroys a $\mathrm{LH}(\mathrm{RH})$ neutrino and creates a $\mathrm{RH}(\mathrm{LH})$ antineutrino.
} 
In fact weak interactions mediated by the $W^{ \pm}$bosons only couple to left-handed particle and right-handed antiparticle states. If chirality were not Lorentz invariant, a $W$ boson could be observed to interact with a particle in some frames but not others - a naively unphysical notion.

Handedness is related to another quantity called helicity. Helicity is the projection of a particle's spin onto its momentum vector. A particle with spin aligned opposite to its momentum has left (negative) helicity. A particle with spin aligned along its momentum vector has right (positive) helicity. For a massive particle, helicity is not Lorentz invariant: an observer can boost to a frame where the momentum of that particle, and thus its helicity, is reversed. In this case, a particle's chirality can be written as a linear combination of its helicity states. However for a massless particle, there is no frame to which an observer can boost that will reverse that particle's helicity. In this case its helicity is invariant and identical to its handedness.

Only left-helicity neutrinos and right-helicity antineutrinos have ever been observed. A natural assumption, and the one included in the Standard Model, is that therefore only lefthanded neutrinos and right-handed antineutrinos exist. Thus the Model predicts massless neutrinos. Since massive neutrinos have been observed experimentally, the Standard Model must be extended to include the missing chiral states. The missing neutrinos will not interact with the $W^{ \pm}$or the $Z^{0}$, and so are called "sterile".

After incorporating these additional neutrino states, a Dirac mass term $(m \bar{\nu} \nu)$ can be constructed using equation 1.1 in analogy with other massive fermions

$$
-m_{D}\left(\bar{\nu}_{L} \nu_{R}+\bar{\nu}_{R} \nu_{L}\right)
$$

where $m_{D}$ is the Dirac mass. This mass term conserves lepton number ${ }^{2}$, and thus in this framework neutrinos and antineutrinos are distinct "Dirac" particles. However if lepton

\footnotetext{
${ }^{2}$ Lepton number is +1 for leptons, -1 for antileptons, and 0 for other particles.
} 
number is not conserved, then the neutrino will be a Majorana particle with neutrino and antineutrino simply being different helicity states of the same particle. Mathematically, this means that the neutrino field is its charge conjugate: $\nu_{L, R}=\nu_{L, R}^{C}$. A Majorana mass term $\left(m \bar{\nu} \nu^{C}\right)$ can then be added to the Larangian using

$$
\nu_{L, R}^{C}=\frac{1}{2}\left(1 \pm \gamma^{5}\right) \nu^{C}=\nu_{R, L}^{C}
$$

to project out the helicity states. The mass term is

$$
-\frac{1}{2} m_{L}\left(\bar{\nu}_{L} \nu_{L}^{C}+\bar{\nu}_{L}^{C} \nu_{L}\right)-\frac{1}{2} m_{R}\left(\bar{\nu}_{R} \nu_{R}^{C}+\bar{\nu}_{R}^{C} \nu_{R}\right)
$$

where $m_{L, R}$ is the mass of the left- (right-) handed neutrino.

If both Dirac and Majorana terms are present, the mass term of the Lagrangian can be written

$$
\mathcal{L}_{\text {mass }}=-\frac{1}{2}\left(\begin{array}{cc}
\bar{\nu}_{L} & \bar{\nu}_{R}^{C}
\end{array}\right)\left(\begin{array}{cc}
m_{L} & m_{D} \\
m_{D} & m_{R}
\end{array}\right)\left(\begin{array}{c}
\nu_{L}^{C} \\
\nu_{R}
\end{array}\right)+\text { h.c. }
$$

then physical masses for the neutrino are determined by diagonalizing the matrix. If $m_{L}=0$ (i.e. the left-handed neutrino - the neutrino of the unmodified Standard Model-derives mass purely from the Dirac term) then the mass eigenvalues are

$$
\lambda_{1} \approx m_{R}, \quad \lambda_{2} \approx \frac{m_{D}^{2}}{m_{R}}
$$

and $m_{D}$ is the geometric mean of $m_{R}$ and $\lambda_{2}$. That means that as one eigenvalue increases the other decreases. This "See-Saw" mechanism explains the very small mass observed for active neutrinos by postulating a very large mass $m_{R}$ for the sterile right-handed ones [11].

\subsection{Neutrino Oscillation Formalism}

The experimental evidence for neutrino mass comes from the preponderance of neutrino oscillation data. This section presents the formalism of neutrino oscillations. All quantities are expressed in natural units $(c=\hbar=1)$ unless explicitly indicated otherwise. 
Massive neutrinos are produced in flavor eigenstates $\left|\nu_{e}\right\rangle,\left|\nu_{\mu}\right\rangle,\left|\nu_{\tau}\right\rangle$ but propagate in mass eigenstates $\left|\nu_{1}\right\rangle,\left|\nu_{2}\right\rangle,\left|\nu_{3}\right\rangle$. The mass eigenstates diagonalize the free neutrino Hamiltonian, and so the flavor states can be written as a linear combination of mass states

$$
\left|\nu_{\alpha}\right\rangle=\sum_{k} U_{\alpha k}\left|\nu_{k}\right\rangle
$$

where the subscript $\alpha$ represents flavor states, the subscript $k$ represents mass states, and $U$ is the unitary "mixing" matrix that relates them.

For a neutrino propagating in time, the flavor state $\left|\nu_{\alpha}\right\rangle_{t}$ can be written

$$
\left|\nu_{\alpha}\right\rangle_{t}=\sum_{k} U_{\alpha k} e^{\left(-i E_{k} t\right)}\left|\nu_{k}\right\rangle
$$

where the $\left|\nu_{k}\right\rangle$ are chosen to be momentum eigenstates and therefore have different energies via $E_{k}^{2}=p^{2}+m_{k}^{2}$. The amplitude to observe a neutrino in the flavor state $\left|\nu_{\beta}\right\rangle$ given that it was initially created in the state $\left|\nu_{\alpha}\right\rangle$ is

$$
\left\langle\nu_{\beta} \mid \nu_{\alpha}\right\rangle_{t}=\sum_{k} U_{\alpha k} U_{\beta k}^{*} e^{\left(-i E_{k} t\right)}
$$

So the probability is

$$
P_{\alpha \rightarrow \beta}=\left|\left\langle\nu_{\beta} \mid \nu_{\alpha}\right\rangle_{t}\right|^{2}=\sum_{k, l} U_{\alpha k} U_{\beta k}^{*} U_{\alpha l}^{*} U_{\beta l} e^{\left(-i\left(E_{k}-E_{l}\right) t\right)}
$$

In the ultrarelativistic limit $\left(\gamma=1 / \sqrt{1-v^{2} / c^{2}}>>1 \text { where } v \text { is the neutrino velocity }\right)^{3}$

$$
E_{k}=\sqrt{p^{2}+m_{k}^{2}} \simeq p+\frac{m_{k}^{2}}{2 p} \approx E+\frac{m_{k}^{2}}{2 E} \quad \text { and } \quad L \simeq t
$$

where $L$ is the distance travelled in time $t$. Then the probability becomes

$$
P_{\alpha \rightarrow \beta}=\left|\left\langle\nu_{\beta} \mid \nu_{\alpha}\right\rangle_{t}\right|^{2}=\sum_{k, l} U_{\alpha k} U_{\beta k}^{*} U_{\alpha l}^{*} U_{\beta l} e^{\left(-i \Delta m_{k l}^{2} L / 2 E\right)}
$$

where $\Delta m_{k l}^{2}=m_{k}^{2}-m_{l}^{2}$.

\footnotetext{
${ }^{3}$ For neutrinos with mass $<1 \mathrm{eV}$ and energy $>1 \mathrm{MeV}, \gamma>10^{6}$.
} 
In the case of two-neutrino oscillations, the mixing matrix is written

$$
U=\left(\begin{array}{cc}
\cos \theta & \sin \theta \\
-\sin \theta & \cos \theta
\end{array}\right)
$$

where $\theta$ is the neutrino mixing angle. Then the probability of observing, for example, an electron neutrino that was originally created as a muon neutrino is

$$
P_{\mu \rightarrow e}=\sin ^{2} 2 \theta \sin ^{2} \frac{\Delta m^{2} L}{4 E} .
$$

In SI units, this is

$$
P_{\mu \rightarrow e}=\sin ^{2} 2 \theta \sin ^{2}\left(1.27 \frac{\Delta m^{2}\left(\mathrm{eV}^{2}\right) L(\mathrm{~m})}{2 E(\mathrm{MeV})}\right)
$$

In general, the full $3 \times 3$ mixing matrix for all neutrino flavors (called the MNS matrix) must be used in order to exactly calculate neutrino oscillation probabilities:

$$
\begin{gathered}
U_{M N S}=\left(\begin{array}{ccc}
U_{e 1} & U_{e 2} & U_{e 3} \\
U_{\mu 1} & U_{\mu 2} & U_{\mu 3} \\
U_{\tau 1} & U_{\tau 2} & U_{\tau 3}
\end{array}\right)= \\
\left(\begin{array}{ccc}
1 & 0 & 0 \\
0 & c_{23} & s_{23} \\
0 & -s_{23} & c_{23}
\end{array}\right)\left(\begin{array}{ccc}
c_{13} & 0 & s_{13} e^{-i \delta} \\
0 & 1 & 0 \\
-s_{13} e^{-i \delta} & 0 & c_{13}
\end{array}\right)\left(\begin{array}{ccc}
c_{12} & s_{12} & 0 \\
-s_{12} & c_{12} & 0 \\
0 & 0 & 1
\end{array}\right)\left(\begin{array}{ccc}
e^{i \beta_{1}} & 0 & 0 \\
0 & e^{i \beta_{2}} & 0 \\
0 & 0 & 1
\end{array}\right)
\end{gathered}
$$

where $s_{i j}=\sin \theta_{i j}$ and $c_{i j}=\cos \theta_{i j}, \theta_{i, j}$ is the mixing angle between flavors $i$ and $j$, the Dirac phase $\delta$ is zero if $\mathrm{CP}$ is preserved, and the $\beta_{1}$ and $\beta_{2}$ phases are zero if neutrinos are not Majorana particles [12]. Then the probability for a neutrino $\alpha$ to oscillate to a neutrino $\beta$ becomes:

$$
P_{\alpha \rightarrow \beta}=\delta_{\alpha \beta}-4 \sum_{l>k} R e\left(U_{\alpha k} U_{\beta k}^{*} U_{\alpha l}^{*} U_{\beta l} \sin ^{2} \frac{\Delta m_{l k}^{2} L}{4 E}\right)+2 \sum_{l>k} \operatorname{Im}\left(U_{\alpha k} U_{\beta k}^{*} U_{\alpha l}^{*} U_{\beta l} \sin \frac{\Delta m_{l k}^{2} L}{2 E}\right)
$$


where the imaginary sum is zero if $\delta=0$ (i.e. if CP is preserved).

Although it is more obvious from the two neutrino oscillation case (equation 1.14), the oscillation amplitude is determined by the mixing angles, and the oscillation frequency depends on the $\Delta m_{l k}^{2}$ terms. The parameters $L$ (the distance from neutrino source to measurement) and $E$ (the neutrino energy) also affect the oscillation probability, and from the perspective of neutrino experiments these are at least measurable if not tunable parameters that probe the neutrino oscillation phase space. In general, an experiment chooses $L / E$ to maximize the oscillation probability for a particular neutrino oscillation mode of interest.

\subsection{Oscillation Landscape}

There are two classes of oscillation experiment: appearance and disappearance. An appearance experiment looks for a neutrino flavor that was not originally created in the source to determine $P_{\alpha \rightarrow \beta}$. A disappearance experiment measures the flavor of neutrinos that were originally present in the source $\left(P_{\alpha \rightarrow \alpha}\right)$, and if oscillations have occured, should observe fewer than expected.

Figure 1.3 illustrates the $\Delta m^{2}$ vs $\sin ^{2} 2 \theta$ oscillation landscape. There are three signal regions: solar, atmospheric, and LSND. The regions are named after the types of experiment that first saw the oscillation signal.

The first solar neutrino oscillation experiments were disappearance experiments. The very first, proposed by Ray Davis Jr. and John Bahcall [13], was designed to study the nuclear reactions that fuel the sun by looking for the electron neutrinos produced in those reactions. They saw only $\sim 1 / 3$ of the predicted number of neutrinos. Other experiments verified fewer measured neutrinos than expected including GALLEX, SAGE, and Super-K (all of which measured solar $\nu_{e}$ directly) as well as KamLAND (which detected $\bar{\nu}_{e}$ from nuclear reactors in the same oscillation region as the solar neutrino signal) [14 17]. 


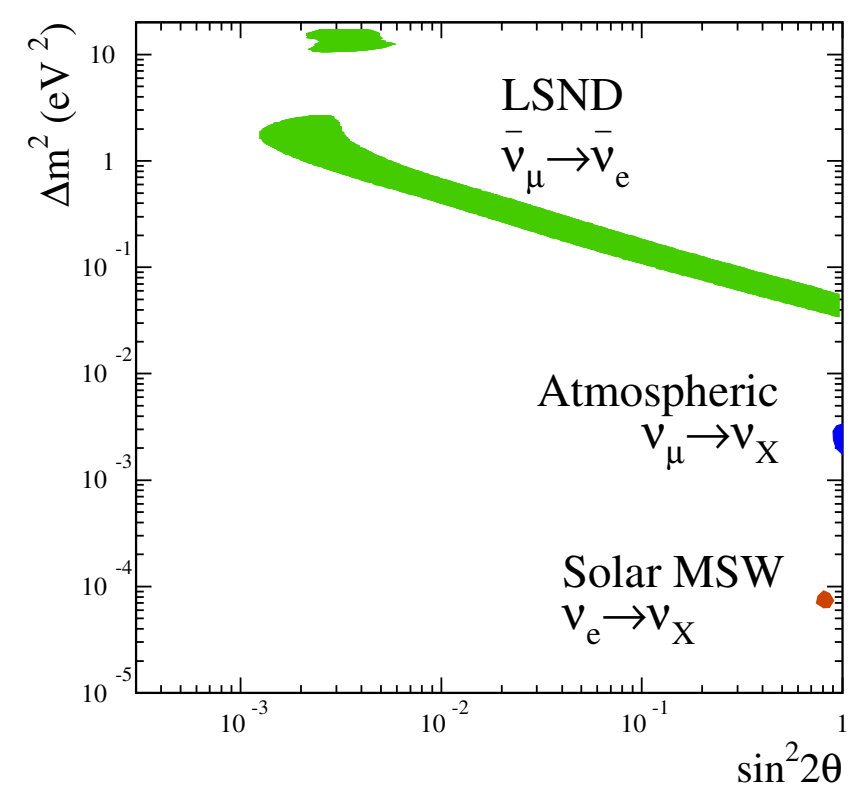

Figure 1.3: Measured oscillation regions in a log-log plot of $\Delta m^{2}$ vs $\sin ^{2} 2 \theta$ space.

The "smoking gun" confirmation that solar neutrinos oscillate came from SNO in 2002 [18. In general, oscillation experiments tag the neutrino flavor using the outgoing lepton from charged-current interactions. In order to interact via this mechanism, the incoming neutrinos must have enough energy to produce the appropriate flavor lepton (electron $m_{e}=$ $0.511 \mathrm{MeV}$, muon $m_{\mu}=106 \mathrm{MeV}$, or tau $m_{\tau}=1800 \mathrm{MeV}$ ). The solar neutrino energy spectrum tops out at $\sim 20 \mathrm{MeV}$ which is not enough energy to produce either a $\mu$ or a $\tau$. However, SNO measured not only the charged-current interactions, but also neutral current scattering from deuteron which is sensitive to all neutrino flavors. The solar neutrino flux determined by the neutral current measurement was in agreement with the nuclear model prediction.

Like their solar counterparts, most atmospheric oscillation experiments have been neutrino disappearance measurements. Experiments such as Kamiokande and Super-K [19, 20] 
find a deficit in the flux of muon neutrinos produced in the atmosphere. These detectors can observe neutrinos created both in the atmosphere above them as well as those generated on the other side of the world that travel through the earth to be detected. Although naively the neutrino fluxes should be the same in both directions, fewer $\nu_{\mu}$ are observed via the longer pathlengths through the earth. These results have been verified by accelerator neutrino experiments such as K2K and MINOS [21, 22] which probe the same "atmospheric" oscillation phase space.

The only unconfirmed signal in figure 1.3 comes from the Liquid Scintillator Neutrino Detector (LSND). This experiment observed the appearance of $\bar{\nu}_{e}$ in a beam of $\bar{\nu}_{\mu}$ from the decay of stopped muons: $\mu^{+} \rightarrow e^{+} \nu_{e} \bar{\nu}_{\mu}$. LSND ran in the $800 \mathrm{MeV}$ LANSCE proton beam at Los Alamos for over 6 calendar years. The beam produced $\sim 30 \mathrm{MeV}$ neutrinos that travelled $\sim 30 \mathrm{~m}$ to the detector. The signature for a $\bar{\nu}_{e}$ event was an inverse beta decay interaction: $\bar{\nu}_{e} p \rightarrow e^{+} n$. The detector triggered on the coincidence of the positron and a delayed $2.2 \mathrm{MeV}$ photon produced from neutron capture. The resulting measurement of events over background $87.9 \pm 22.4 \pm 6.0(3.8 \sigma)$ was interpreted as $\bar{\nu}_{\mu} \rightarrow \bar{\nu}_{e}$ oscillations [23].

When the LSND result is combined with the solar and atmospheric sectors, the three signals are not compatible with only three neutrino flavors unless non-standard physics such as CPT violation or Lorentz-invariance violation is invoked. If there are only three flavors of neutrino (measurements show that only three flavors interact via the weak force [24]), then

$$
\Delta m_{13}^{2}=\Delta m_{12}^{2}+\Delta m_{23}^{2}
$$

However, the three measured $\Delta m^{2}$ from figure 1.3 do not add in this way. Since the LSND signal has not been confirmed, it is important to verify this experimentally before embracing new physics. 


\subsection{MiniBooNE Results}

MiniBooNE was proposed in 1997 to test the LSND oscillation result. MiniBooNE looks for an excess of $\nu_{e}$ in a beam of $\nu_{\mu}$ which should produce the same result as LSND if CPT is preserved. MiniBooNE also produces neutrinos at higher energies $(\sim 550 \mathrm{MeV})$ and has a longer baseline $(\sim 550 \mathrm{~m})$ than LSND but preserves its $L / E$. The other main difference is the event signature: MiniBooNE looks for the charged-current interaction of $\nu_{e}$ in a nucleus $\left(\nu_{e} n \rightarrow e^{-} p\right)$. The detector triggers on the Cherenkov ring produced by the electron in the detector medium. Further details of the experiment are discussed in Chapters 3 and 4.

In order to prevent uninentional biases from being introduced to the data analysis, MiniBooNE adopted a blindness policy. The effect of this was that data in the oscillation region predicted by LSND was made inaccessable to all but the most basic detector quality algorithms: "closed" in a "blindness box." The oscillation analysis was then based on Monte Carlo simulations and various internal and external sources of data to constrain and measure parameters of importance to the result. Once finalized, vetted, and approved on samples of fake data, a rigorous un-blinding proceedure was followed to "open the box" real-time in front of the MiniBooNE collaboration. This event occured on March 27, 2007.

MiniBooNE employed two largely independent analyses to examine the data. The main analysis utilized a track-based (TB) reconstruction algorithm [26] to reconstruct and identify events. A secondary analysis applied a boosted decision tree (BDT) algorithm [27] to identify and separate electron candidate events. The track-based analysis predicted $358 \pm 19$ (stat) \pm 35 (syst) electron events from the Monte Carlo in the energy range $475<E_{\nu}<1250 \mathrm{MeV}$ and observed 380 events in the data. The BDT analysis predicted $1070 \pm 33($ stat $) \pm 225$ (syst) events in the energy range $300<E_{\nu}<1600 \mathrm{MeV}$ and observed 971 events in the data. Neither observed a significant excess of $\nu_{e}$ events above background in these energy ranges putting a limit on the allowed oscillation parameter space as shown in figure 1.4 consistent with no oscillations assuming a two-neutrino, 


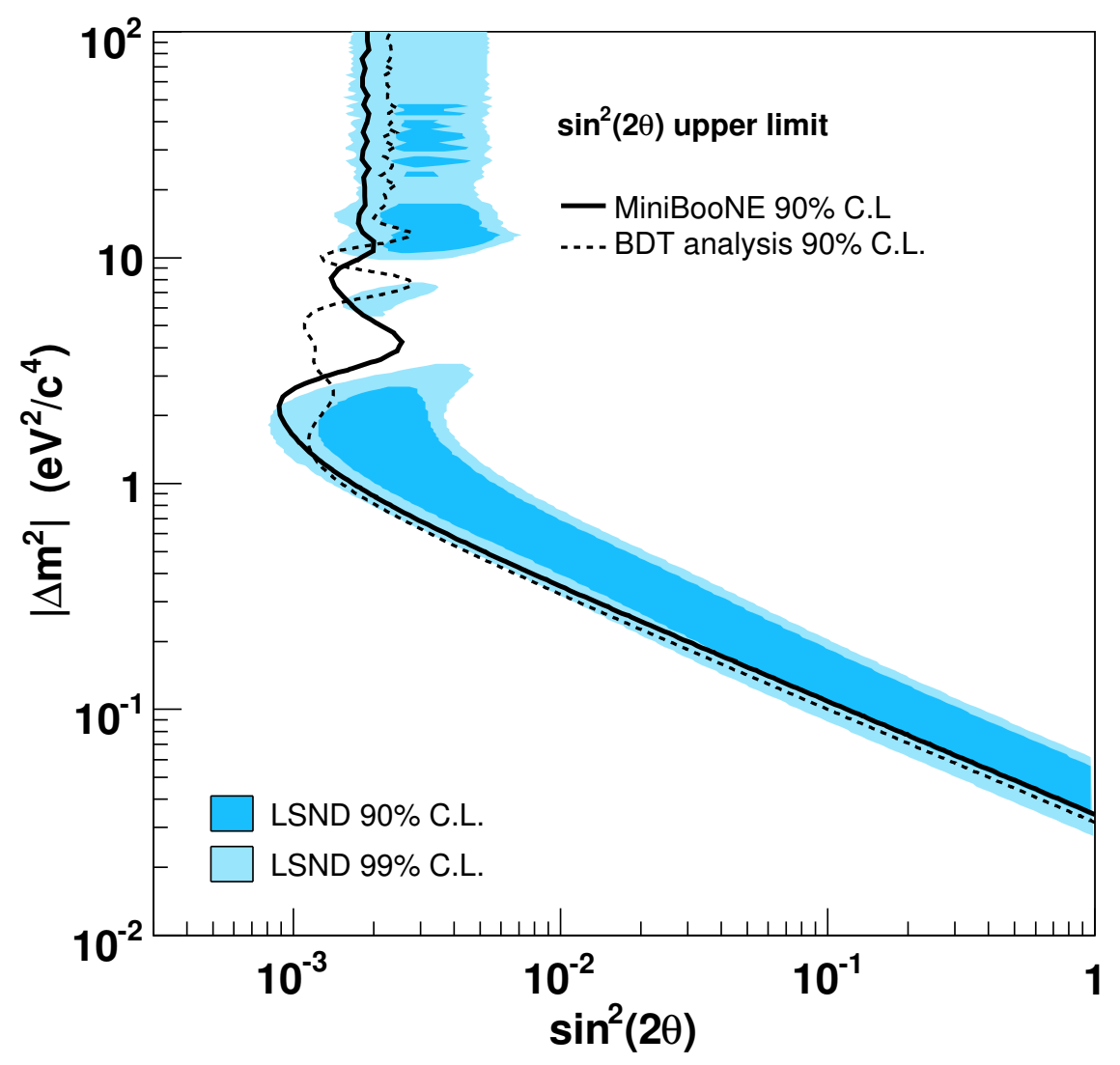

Figure 1.4: Measured 90\% confidence limit for MiniBooNE analyses. The track-based result is called "MiniBooNE" here since it is the primary result [25]. 
appearance-only oscillation model $[25]$.

\title{
1.6 Synopsis
}

\begin{abstract}
Although neutrino oscillation measurements are the main goal of MiniBooNE, there are many other ongoing analyses of MiniBooNE data, in particular extractions of neutrino cross sections. The remainder of this dissertation presents the cross section for neutral-current neutrino-nucleon elastic scattering. Chapter 2 outlines the motivation for measuring this channel. Chapter 3 presents the experimental set-up of MiniBooNE from initial protons to data on tape. Chapter 4 describes the simulations that model events and the algorithms that reconstruct both the simulated and actual events. Chapter 5 explains the selections used to isolate the sample of neutral current elastic events used in this analysis. And Chapter 6 presents the procedures that yield the neutral-current neutrino-nucleon elastic cross section results as well as the results themselves.
\end{abstract}




\section{Chapter 2}

\section{Neutral Current Elastic Cross Sections}

Neutral current nucleon elastic scattering occurs when a neutrino transfers momentum to a nucleon via a $Z^{0}$ boson: $\nu N \rightarrow \nu N$. This interaction has been used to investigate weak interactions, probe nucleon structure, study neutrino oscillations, and has been proposed to measure the spectrum of neutrinos from supernovae. This Chapter presents an outline of neutrino-nucleon elastic scattering in theory and experiment.

\subsection{History}

Proposed in electroweak unified theories in the 1960's [28 30], neutral current interactions were first observed in 1973 by the Gargamelle bubble chamber at CERN [31, 32]. Out of $\sim 1$ million total pictures of $\nu_{\mu}$ and $\bar{\nu}_{\mu}$ events, this experiment saw a single elastic electron event $\nu e^{-} \rightarrow \nu e^{-}$and $\sim 160$ neutral current nucleon deep-inelastic events $\nu N \rightarrow \nu X$ (where $X$ is a complex hadronic final state). 
These results caused much excitement in the particle physics community prompting measurements of a variety of "muon-less" interactions such as neutral current elastic (NCE) $\nu N \rightarrow \nu N$. This channel was first observed as $\nu(\bar{\nu}) p \rightarrow \nu(\bar{\nu}) p$ by the Columbia-IllinoisRockefeller (CIR) [33] and Harvard-Pennsylvania-Wisconsin (HPWB) [34 36] collaborations at Brookhaven National Laboratory (BNL) and confirmed later by other groups [37, 38]. Although they only measured small numbers of events (ranging from 20 to 100), these experiments were able to place bounds on electroweak theories by constraining $\sin ^{2} \theta_{W}$ (where $\theta_{W}$ is the weak mixing angle).

After more runs to amass higher statistics ( $200 \nu_{\mu}$ events), members of the HPBW collaboration showed that the Lorentz struture of the neutral weak current (how the $Z^{0}$ interacts with other particles) is vector minus axial-vector like the charged weak current [39]. In other words, the neutral current also violates parity ${ }^{1}$

The next step was to investigate the nucleon neutral weak current: how the $Z^{0}$ interacts specifically with the nucleon. This current is parametrized with form factors (discussed in the next section) that describe the structure of the nucleon and are of much interest in nuclear physics. Both the high statistics HPWB experiment and another experiment at BNL [40] extracted form factors. However their results left questions about the nature of strange $(s)$ quarks in the nucleon that have led to proposals by modern experiments for clarification (discussed in section 2.5).

Other uses for neutral currents include neutrino oscillation experiments and measurements of supernova flux. The neutral current reaction $\nu_{x} d \rightarrow \nu_{x} p n$ (where $x$ can represent any flavor of neutrino) was used by the SNO experiment to confirm the solar nuclear physics model and thus conclusively show solar neutrino oscillation [41]. It has also been noted that

\footnotetext{
${ }^{1}$ The weak charged current is $\propto \gamma^{\mu}\left(1-\gamma^{5}\right)$, which is pure $V-A$ (vector minus axial-vector) and so maximally violates parity. The weak neutral current is $\propto \gamma^{\mu}\left(c_{V}^{f}-c_{A}^{f} \gamma^{5}\right)$ which is $V-A$ in structure, but modified by the coefficients $c_{V}^{f}$ and $c_{A}^{f}$ which depend on the fermion $f$ involved in the interaction (for Standard Model neutrinos $\left.c_{V}^{f}=c_{A}^{f}=1 / 2\right)$.
} 


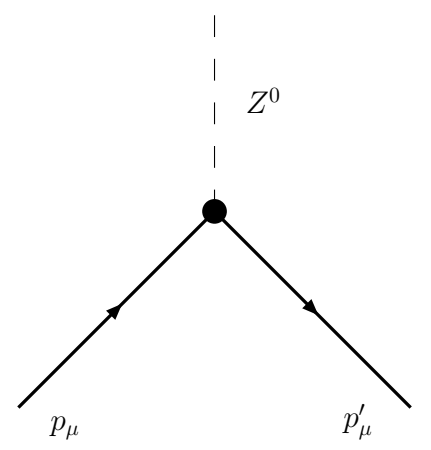

Figure 2.1: Depiction of the neutral weak nucleon vertex.

this insensitivity to neutrino flavor makes NCE interactions ideal for measuring neutrino spectra from nearby supernovae since most of that flux is $\nu_{\mu}$ and $\nu_{\tau}$ [42, 43]. Several proposals have been made to measure these events in existing detectors (e.g. [44, 45]).

The work presented here focuses on using neutrinos to measure nucleon structure. In order to understand how this is possible, the formalism of the nucleon neutral weak current is presented next.

\subsection{Nucleon Neutral Weak Current}

The diagram in figure 2.1 illustrates the neutral weak interaction with the nucleon. The current for this interaction can be written

$$
J_{\mu}=\left\langle N\left(p^{\prime}\right)\left|F_{1}\left(Q^{2}\right) \gamma_{\mu}+F_{2}\left(Q^{2}\right) \sigma_{\mu \nu} q^{\nu}+G_{A}\left(Q^{2}\right) \gamma_{\mu} \gamma_{5}\right| N(p)\right\rangle
$$

where $F_{1}\left(Q^{2}\right), F_{2}\left(Q^{2}\right)$, and $G_{A}\left(Q^{2}\right)$ are the nucleon form factors and $Q^{2}$ is related to the four-momentum transferred to the nucleon $q^{\nu}$ by

$$
Q^{2}=-\left(q^{\nu}\right)^{2}=-\left(p^{\nu \prime}-p^{\mu}\right)^{2}
$$


The first two terms in the current are the vector contribution where $F_{1}\left(Q^{2}\right)$ and $F_{2}\left(Q^{2}\right)$ are respectively the Dirac and Pauli form factors. The last term in the current is the axial current where $G_{A}\left(Q^{2}\right)$ is the nuclear axial form factor.

The $Q^{2}$ dependence of the form factors is typically parametrized using a dipole form. As an example, the $Q^{2}$ dependence of the axial form factor is given here

$$
G_{A}\left(Q^{2}\right)=\frac{1}{2} \frac{G_{A}(0)}{\left(1+Q^{2} / M_{A}^{2}\right)} \tau_{3}+G_{A}^{s}\left(Q^{2}\right)
$$

where $G_{A}(0)$ is precisely determined from beta decay measurements, $M_{A}$ is the dipole cutoff mass, $\tau_{3}$ is $+1(-1)$ for proton (neutron) scattering, and a term due to the contribution from strange quarks has been explicitly introduced.

Experiments have shown that strange quarks in the nucleon quark sea contribute to nucleon mass and momentum (e.g. [46 48]). It is therefore important to consider possible strange quark contributions to the neutral weak nucleon current. These contributions enter the current as the additional form factors: $F_{1}^{s}\left(Q^{2}\right), F_{2}^{s}\left(Q^{2}\right)$, and $G_{A}^{s}\left(Q^{2}\right)$. The $Q^{2}$ evolution of these form factors can also be parameterized by a dipoles:

$$
\begin{aligned}
F_{1}^{s}\left(Q^{2}\right) & =\frac{1}{6} \frac{-r_{s}^{2} Q^{2}}{\left(1+Q^{2} / M_{1}^{1}\right)^{2}} \\
F_{2}^{s}\left(Q^{2}\right) & =\frac{\mu_{s}}{\left(1+Q^{2} / M_{2}^{2}\right)^{2}} \\
G_{A}^{s}\left(Q^{2}\right) & =\frac{1}{2} \frac{\Delta s}{\left(1+Q^{2} / M_{A}^{2}\right)}
\end{aligned}
$$

where $M_{1}$ and $M_{2}$ are the relevant masses of the strange vector form factors ${ }^{2} r_{s}$ is the strange radius of the nucleon (analogous to the nucleon charge radius), $\mu_{s}$ is the strange anomalous magnetic moment of the nucleon, and $\Delta s$ is the component of nucleon spin carried by strange quarks.

Much effort has been spent to understand the structure of the nucleon through measurements of its strange and non-strange form factors with both electron and neutrino scattering experiments. The results of the electron exeriments are discussed next.

\footnotetext{
${ }^{2}$ These masses are commonly set to be equal to the vector cutoff mass $M_{V}: M_{1}=M_{2}=M_{V}$.
} 


\subsection{Measuring Nucleon Structure with Electrons}

Electrons interact via both the weak and electromagnetic forces. The electromagnetic coupling (charge) of electrons makes generating and characterizing intense, focused, polarized electron beams a reasonable task. Because these beams readily interact, scattering of polarized electrons on nuclear targets allows high precision measurements of nucleon form factors.

In the simplest case, the non-strange terms of the weak vector form factors $F_{1}\left(Q^{2}\right)$ and $F_{2}\left(Q^{2}\right)$ are directly related to their electromagnetic counterparts ${ }^{3} F_{1}^{E M}\left(Q^{2}\right)$ and $F_{2}^{E M}\left(Q^{2}\right)$ under the conserved vector current (CVC) hypothesis. CVC assumes that, like electromagnetism, the weak vector current is conserved with a universal coupling constant. A consequence of this hypothesis is that it relates the weak to the electromagnetic current via isospin 4 .

$$
F_{i}=\left(\frac{1}{2}-\sin ^{2} \theta_{W}\right)\left(F_{i, p}^{E M}-F_{i, n}^{E M}\right) \tau_{3}-\sin ^{2} \theta_{W}\left(F_{i, p}^{E M}+F_{i, n}^{E M}\right)-\frac{1}{2} F_{i}^{s}
$$

where $i$ is 1 or $2, F_{i}^{s}$ is the strange contribution to the vector form factors, and the $Q^{2}$ dependence of the form factors is implied. Polarized electron scattering experiments such as BLAST and CLAS measure linear combinations and ratios of the electromagnetic form factors separately for protons and neutrons to high precision $[50-52]$.

Although the strange quark terms $F_{1}^{s}\left(Q^{2}\right)$ and $F_{2}^{s}\left(Q^{2}\right)$ do not have electromagnetic complements, they can be extracted from parity violating electron scattering (PVES) experiments. These derive sensitivity to the neutral weak form factors via quantum interference between the electromagnetic and weak interactions (figure 2.2). Thus collaborations such as SAMPLE, PVA4, G0, and HAPPEX are able to place tight constraints on the strange

\footnotetext{
${ }^{3}$ The electromagnetic current is pure vector: $J_{\mu}^{E M}=\left\langle N\left(p^{\prime}\right)\left|F_{1}^{E M}\left(Q^{2}\right) \gamma_{\mu}+F_{2}^{E M}\left(Q^{2}\right) \sigma_{\mu \nu} q^{\nu}\right| N(p)\right\rangle$.

${ }^{4}$ The relationship is $\left\langle N^{\prime}\left|J_{\mu}^{Z}\right| N\right\rangle=\left\langle N^{\prime}\left|J_{\mu}^{3}-2 \sin ^{2} \theta_{W} \cdot J_{\mu}^{E M}\right| N\right\rangle$ where $J_{\mu}^{3}$ is the third component of the isospin current [49].
} 

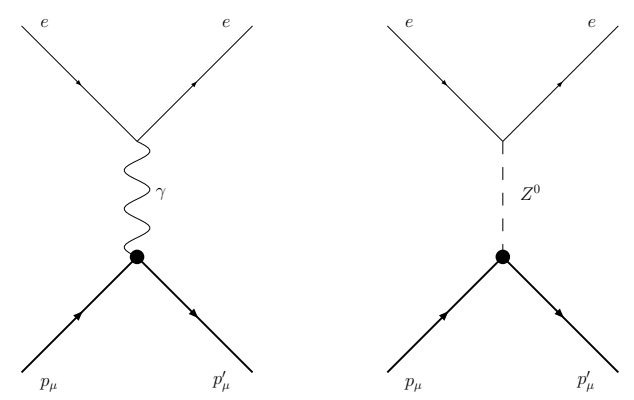

Figure 2.2: These electron scattering amplitudes interfere to provide sensitivity to the neutral weak current for PVES.

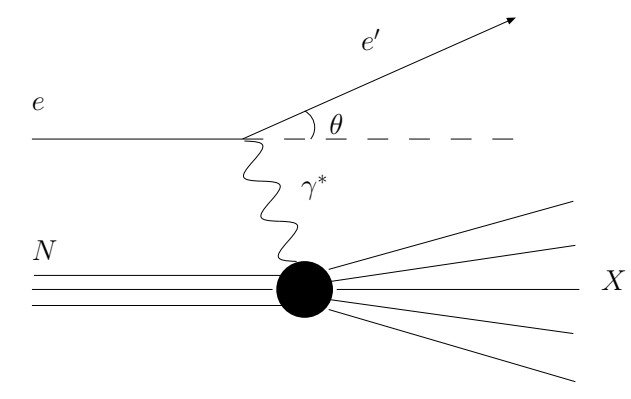

Figure 2.3: Schematic figure of a DIS process where $X$ is a complex hadronic final state.

vector form factors. The most recent data indicate strangeness contributions to the form factors that are small, positive, and consistent with zero [53 56].

Polarized electron scattering experiments can also claim to measure the axial form factors of the weak neutral nucleon current. Using high energy electrons, deep inelastic scattering (DIS) experiments such as the SMC break apart (shatter) polarized nucleons and analyze the outgoing electron (figure 2.3). The polarized nucleon structure function $g_{1}$ is extracted from this data as a function of $Q^{2}$ and $x$ (the fraction of momentum carried by 
the electron) $5^{5}$. This function can be used in conjunction with the assumption of SU(3) flavor symmetry and measurments of hyperon beta decays to determine the quark spin contributions $\Delta u, \Delta d$ and $\Delta s$ which are related to $G_{A}^{s}\left(Q^{2}\right)$ [57]. Recently, semi-inclusive DIS (SIDIS) experiments such as HERMES have verified and improved on the results from DIS. These experiments are similar with the addition that mesons in the final state $X$ are measured directly, reducing one of the complications of DIS. Values for the quark spin components reported by the SMC [58] have been confirmed to higher precision by the most recent HERMES results: $\Delta u=0.842 \pm 0.013, \Delta d=-0.427 \pm 0.013$, and $\Delta s=-0.085 \pm 0.018$ [48].

With the exception of the electromagnetic form factors, obtaining nucleon structure information from electron scattering experiments is not direct. However, neutrino elastic and quasi-elastic scattering from nucleons directly probe the weak structure of the nucleon. The next section presents the kinematics of these interactions and the form of the cross section.

\subsection{Neutrino-Nucleon Elastic Scattering}

Neutral current elastic (NCE) scattering is the simplest interaction of a neutrino with a nucleon. The next most simple is charged current quasi-elastic (CCQE) scattering in which a $W$ boson changes the flavor of the lepton and the isospin of the nucleon. Kinematically, these interactions can be treated identically, and from the perspective of the nucleon vertex, the observables are the same.

In the lab frame (nucleon initially at rest), the four-momenta of each particle in the

\footnotetext{
${ }^{5}$ Specifically, $g_{1}(x)=\frac{1}{2} \sum_{q} e_{q}^{2} \Delta q(x)$ where $e_{q}$ is the charge distribution function for quarks of flavor $q$, and $\Delta q(x)$ is a polarized quark distribution function (parallel minus anti-parallel).
} 

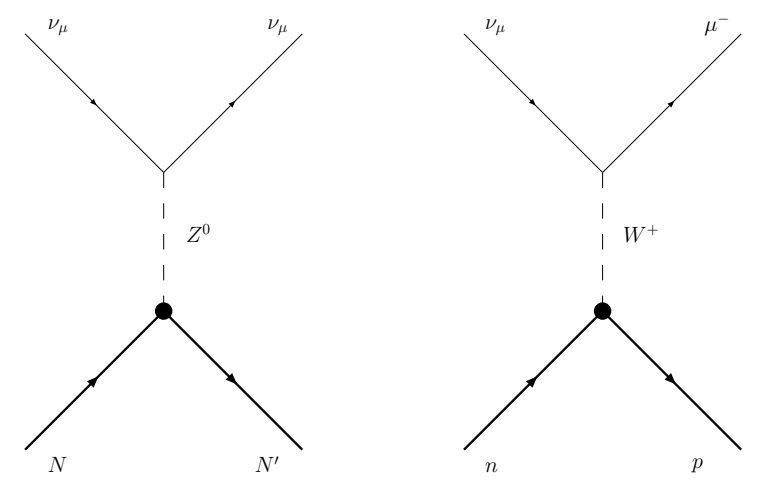

Figure 2.4: Diagrams for NCE and CCQE scattering with muon neutrinos.

interaction (figure 2.4) is

$$
\begin{aligned}
& q_{1}=\left(E_{\nu}, \vec{p}_{\nu}\right) \\
& q_{2}=\left(E_{l}, \vec{p}_{l}\right) \\
& p_{1}=\left(M_{N}, 0\right) \\
& p_{2}=\left(E_{N}, \vec{p}_{N}\right)
\end{aligned}
$$

where $E_{l}$ and $\vec{p}_{l}$ are the energy and momentum of the outgoing lepton $\left(\nu_{\mu}\right.$ for NCE and $\mu$ for CCQE). It is useful to consider the Mandlestam kinematic variables of the interaction 49]

$$
\begin{aligned}
s & =\left(p_{1}+q_{1}\right)^{2}=\left(p_{2}+q_{2}\right)^{2}=M_{N}^{2}+2 M_{N} E_{\nu} \\
t & =\left(p_{2}-p_{1}\right)^{2}=\left(q_{2}-q_{1}\right)^{2}=-Q^{2} \\
u & =\left(q_{2}-p_{1}\right)^{2}=\left(p_{2}-q_{1}\right)^{2}=M_{N}^{2}-2 M_{N} E_{l}
\end{aligned}
$$

where the outgoing lepton mass is assumed to vanish. The negative of the kinematic variable $t$ is $Q^{2}$ which, solving for $\left(p_{2}-p_{1}\right)^{2}$ can be simply described by the proton kinetic energy

$$
Q^{2}=2 M_{N} T_{N}
$$


Given the angle of the outgoing nucleon with respect to the incident neutrino, it is also possible to determine the neutrino energy

$$
E_{\nu}=\frac{M_{N}}{\left(1+2 M_{N} / T_{N}\right)^{1 / 2} \cos \theta_{N}-1} .
$$

The differential cross section as a function of $Q^{2}$ for these interactions is found using (reference [59])

$$
\frac{d \sigma}{d Q^{2}}=\frac{1}{64 \pi E_{\nu}^{2} M_{N}^{2}} \cdot \overline{|\mathcal{M}|^{2}}
$$

where the elements of the matrix $\mathcal{M}$ come from from the nucleon weak current (equation 2.1. Following the formalism of Llewellyn-Smith [60], the cross section can be written

$$
\frac{d \sigma}{d Q^{2}}=\frac{M_{N}^{2} G_{F}^{2}}{8 \pi E_{\nu}^{2}}\left[A\left(Q^{2}\right) \pm B\left(Q^{2}\right) \frac{(s-u)}{M_{N}^{2}}+C\left(Q^{2}\right) \frac{(s-u)^{2}}{M_{N}^{4}}\right]
$$

where the + in front of the $B\left(Q^{2}\right)$ term is for neutrinos and the - for antineutrinos, $G_{F}$ is the Fermi constant, $s-u=4 M_{N} E \nu-Q^{2}$ from the Mandelstam variables, and the coefficients $A\left(Q^{2}\right), B\left(Q^{2}\right)$, and $C\left(Q^{2}\right)$ contain the form factors:

$$
\begin{gathered}
A\left(Q^{2}\right)=\frac{Q^{2}}{M_{N}^{2}}\left[G_{A}^{2}\left(1+\frac{Q^{2}}{4 M_{N}^{2}}\right)-F_{1}^{2}\left(1-\frac{Q^{2}}{4 M_{N}^{2}}\right)+F_{2}^{2}\left(1-\frac{Q^{2}}{4 M_{N}^{2}}\right) \frac{Q^{2}}{4 M_{N}^{2}}+F_{1} F_{2} \frac{Q^{2}}{M_{N}^{2}}\right] \\
B\left(Q^{2}\right)=\frac{Q^{2}}{M_{N}^{2}} G_{A}\left(F_{1}+F_{2}\right) \\
C\left(Q^{2}\right)=\frac{1}{4}\left[G_{A}^{2}+F_{1}^{2}+F_{2}^{2} \frac{Q^{2}}{4 M_{N}^{2}}\right]
\end{gathered}
$$

where the $Q^{2}$ dependence of the form factors has not been written explicitly. Note that at low $Q^{2}$ the $C\left(Q^{2}\right)$ term dominates the cross section and is very sensitive to the axial term.

All of the nucleon structure information is contained in the form factors of the neutrino elastic differential cross section. The following section describes both past and proposed measurements of neutrino scattering and how they extract nucleon structure information. 


\subsection{Measuring Nucleon Structure with Neutrinos}

Neutral current elastic scattering of neutrinos from protons is ideal for studying the weak structure of the nucleon. As illustrated in the previous section, the cross section is well understood. Furthermore unlike electron scattering experiments, there are no model dependencies, assumptions of flavor SU(3), or interference from the other Standard Model interactions. Experimentally, this channel is completely defined by the measured kinetic energy of the single observable final state particle: the nucleon.

Neutrino scattering at low $Q^{2}$ is particularly sensitive to the axial form factor $G_{A}\left(Q^{2}\right)$. The non-strange part of this form factor depends on $G_{A}(0)$ and $M_{A}$ (equation 2.3). $G_{A}(0)$ is well know from neutron beta decay, but the axial mass $M_{A}$ is less well known. Given the vector form factors, $F_{1}$ and $F_{2}$, the axial mass can be extracted directly from both the NCE and CCQE differential cross sections.

Extracting the strange axial form factor $G_{A}^{s}$ generally involves taking ratios of these cross sections. This reduces the sensitivity of the result to experimental uncertainties such as the neutrino flux as well as theoretical uncertainties such as final state interactions. The ratio of the neutrino neutral current proton $(\mathrm{NCp})$ to neutral current neutron $(\mathrm{NCn})$ cross sections, and the ratio of the neutrino NCp to CCQE cross sections are the most common, though others can be employed 61,63$]$.

The first neutrino scattering experiment to explicitly extract nucleon form factors from its data was the high statistics run of the HPWB experiment [39] discussed in section 2.1. This experiment measured both the neutrino and anti-neutrino differential NCp cross sections and solved for all three non-strange nucleon form factors at $Q^{2}=0$ simultaneously. These values can be calculated explicitly using the anomalous magnetic moments of the proton and neutron, $G_{A}(0)$, and $\sin ^{2} \theta_{W}$, though at the time of the experiment not all of these values were well constrained. The results as extracted by this experiment do not agree 


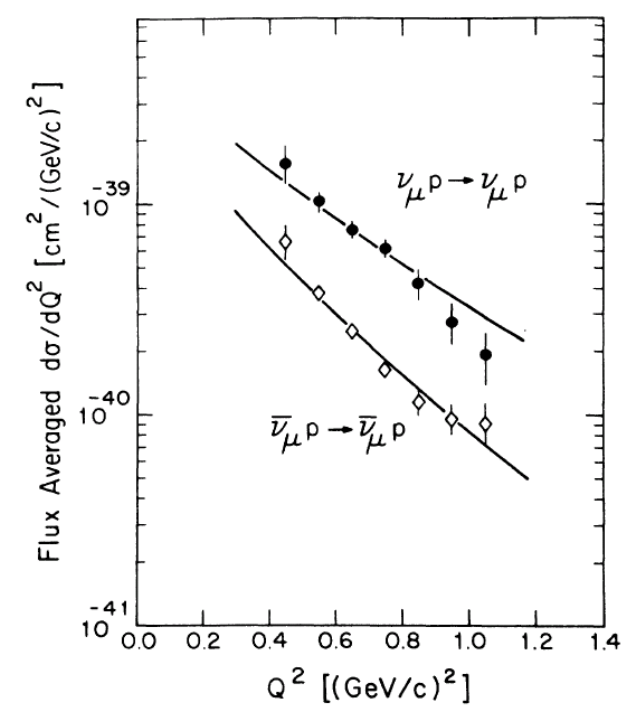

Figure 2.5: BNL E734 differential cross section results for $\nu_{\mu}\left(951\right.$ events) and $\bar{\nu}_{\mu}(776$ events) scattering. The lines are the BNL best fits to their combined data that yield $M_{A}=1.06 \mathrm{GeV}$ [40].

with the calculated form factors using modern values of the above inputs.

The other "high statistics" neutrino experiment at BNL was BNL E734 [40]. This experiment sought to constrain $G_{A}\left(Q^{2}\right)$ via measurements of the flux averaged differential cross section for both $\nu_{\mu}$ and $\bar{\nu}_{\mu}$ scattering (figure 2.5). Their measurement of the axial form factor placed $M_{A}$ (the axial cutoff mass) at $1.06 \pm 0.05 \mathrm{GeV}$ which is also in agreement with the world average. Furthermore, BNL E734 extracted a parameter related to $G_{A}^{s}(0)$ : $\eta=-\Delta s / G_{A}(0)=0.12 \pm 0.07$ which gives a small, negative $\Delta s$ consistent with the DIS results. However, reanalyses of the data [64 66] point to values consistent with zero for that quantity leading to interest in both the experimental and theoretical community for new experiments to measure strange form factors in the nucleon.

One of the proposed experiments, the Fine-grained Intense Neutrino Scattering Scintillator Experiment (FINeSSE) seeks to measure $\Delta s$ to high precision. The estimate for the 
experimental error after $2 \times 10^{20}$ protons on target is \pm 0.025 [67]. FINeSSE proposes to measure neutrino scattering with a novel detector technology involving optical fibers in an open volume of liquid scintillator. A prototype called Scibath is currently being tested at the Indiana University Cyclotron Facility (IUCF).

Minerva [68] also has as one of its main physics goals a precision measurement of the axial form factor. This experiment is a compact scintillator detector under construction at Fermi National Accelerator Laboratory (FNAL) to be placed in the Neutrinos from the Main Injector (NuMI) beamline upstream of the Main Injector Neutrino Oscillation (MINOS) near detector.

Currently running is SciBooNE [69], an experiment in the Booster beamline (the same neutrino beam as MiniBooNE) whose purpose is to collect high statistics cross section data at the neutrino energy range of interest for the Tokai to Kamioka (T2K) long baseline neutrino oscillation experiment. Using the Scibar solid scintillator bar detector, SciBooNE will collect $1 \times 10^{20}$ protons on target in both neutrinos and antineutrinos. An effort is underway to determine the $\mathrm{NCp}$ and CCQE cross sections to extract form factor information, however this analysis is still in its formative stages.

\subsection{Neutral Current Elastics in MiniBooNE}

MiniBooNE is currently running and has collected nearly $1 \times 10^{21}$ protons on target of combined neutrino and antineutrino data. Mature analyses for both NCE and CCQE events have been developed and have extracted nuclear structure information.

Unfortunately, the current MiniBooNE NCE analysis is not able to extract $\Delta s$ from the data. As defined in equation 2.3 , the axial form factor changes sign for proton vs neutron scattering. Therefore, to prevent averaging out the affect of the form factor, an experiment seeking to measure this must be able to distinguish proton and neutron events 
in its detector.

Separating these events is possible in a fine-grained tracking detector due to differences in how neutrons and protons lose energy along their tracks. However, MiniBooNE is an open volume detector, and cannot reconstruct useful track information for low energy particles such as these. Therefore it acts like a calorimeter for protons and neutrons, collecting the total energy deposited by these particles without a method to distinguish them.

Since MiniBooNE will not measure neutral current proton and neutral current neutron cross sections individually, it will measure their sum. This total elastic cross section is sensitive to the axial mass $M_{A}$ of the axial form factor. MiniBooNE has already extracted $M_{A}$ using CCQE events, so it will be interesting to compare the two results.

The remainder of this dissertation describes how MiniBooNE collects, reconstructs, and analyzes neutral current elastic events to determine both $M_{A}$ and measure a high-statistics NCE differential cross section as a function of $Q^{2}$. It begins with an overview of the MiniBooNE experimental setup. 


\section{Chapter 3}

\section{MiniBooNE}

MiniBooNE is a neutrino oscillation experiment at Fermilab designed to test the LSND oscillation signal. Since August 2002, MiniBooNE has collected over 800,000 neutrino events

from $1 \times 10^{21}$ protons on target $\left(\sim 7.5 \times 10^{20}\right.$ in $\nu_{\mu}$ and $\sim 2.5 \times 10^{20}$ in $\left.\bar{\nu}_{\mu}\right)$ comprising the world's largest sample of neutrino interactions at $\sim 1 \mathrm{GeV}$ neutrino energies. This Chapter presents the experimental setup of MiniBooNE.

\subsection{Overview}

The neutrino beam for MiniBooNE begins when $8 \mathrm{GeV}$ kinetic energy protons from the Fermilab Booster are extracted to the MiniBooNE target hall. These protons impinge on a beryllium target inside a magnetic focusing horn and interact to produce (mainly) charged pions. The pions are sign-selected according to their electric charge by the strong magnetic fields in the focusing horn such that pions with the "correct" sign are bent toward the detector. These are then allowed to decay to muons and muon neutrinos in a decay region. Muons and leftover pions are stopped in an absorber at the end of this region so that only a pure neutrino beam reaches the detector. A cartoon overview of the beamline and detector 


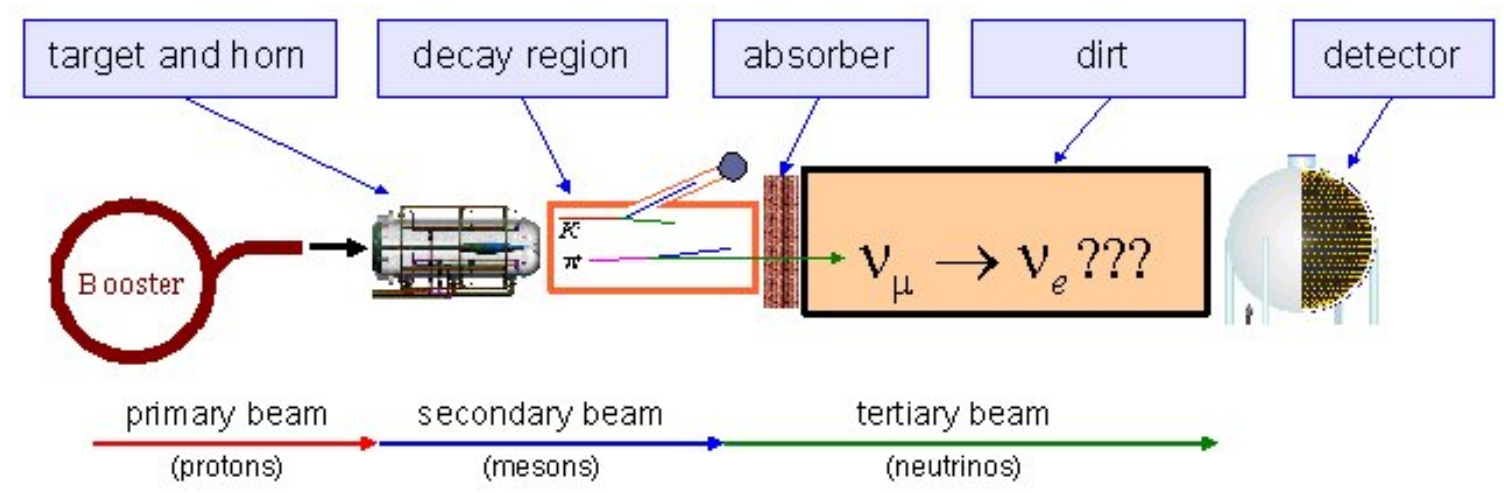

Figure 3.1: A cartoon overview of the MiniBooNE beamline and detector.

is shown in figure 3.1 .

The detector is a $12 \mathrm{~m}$ diameter sphere filled with $\sim 800$ tons of pure (undoped) mineral oil. It stands in an underground cylindrical vault $\sim 550 \mathrm{~m}$ from the target which is covered by a $\sim 3 \mathrm{~m}$ overburden of dirt to reduce the rate of cosmic rays. The total volume of mineral oil is separated into two optically isolated regions: an inner detector region, and an outer veto region. The inner region is instrumented with 1280 photomultiplier tubes (PMTs) that cover $\sim 10 \%$ of the detector surface. The veto region contains 240 PMTs.

The PMTs detect radiation caused by the movement of charged particles in the oil. This radiation can arise from Cherenkov or scintillation processes. The timing and topographic information from this radiation is used to identify and classify neutrino interactions in MiniBooNE.

\subsection{Beam}

The beam used for MiniBooNE consits of three stages: the primary proton beam, a secondary meson beam, and the final neutrino beam. 


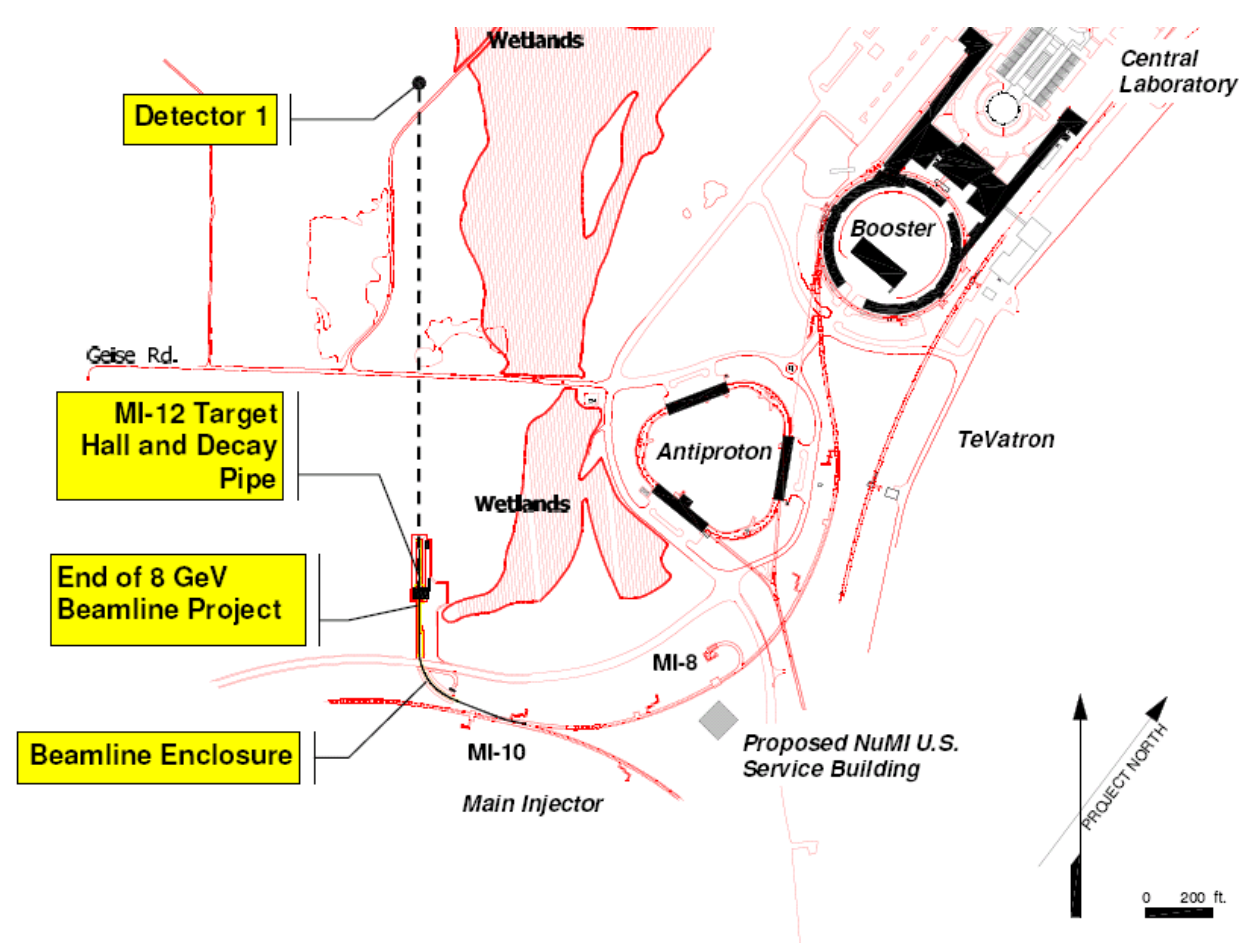

Figure 3.2: Schematic view of the $8 \mathrm{GeV}$ beamline from the Booster to MiniBooNE. 72]

\subsubsection{Primary (Proton) Beam}

The Fermilab Booster, a proton synchrotron, provides the primary beam of $8 \mathrm{GeV}$ kinetic energy $(8.89 \mathrm{GeV} / \mathrm{c}$ momentum) protons. These are extracted to MiniBooNE in pulses of $\sim 4 \times 10^{12}$ at rates of $\sim 3-5 \mathrm{~Hz}[70$. The number of protons in a pulse is measured by two toroids at different locations in the beamline with an uncertainty of $\sim 1 \%$ [71]. Figure 3.2 shows the path of protons from the Booster to MiniBooNE.

As protons travel to the MiniBooNE target, their trajectories are measured by beam position monitors on a pulse-by-pulse basis. A program called Autotune [73] uses this information to automatically adjust the beam position and angle to keep the protons centered on the target. Loss monitors at the target and along the beamline record radiation levels, 
and are used to inhibit beam in case of poor alignment.

\subsubsection{Secondary (Meson) Beam}

Primary protons interact inelastically in the beryllium target to produce mainly charged pions and kaons. These are directed toward (or away from) the detector by magnetic fields in the surrounding focusing horn. Those mesons focused toward the detector comprise the secondary beam.

The MiniBooNE target length is $71 \mathrm{~cm}(\sim 1.7$ interaction lengths) allowing the majority of the proton beam to interact in the beryllium. However its radius is only $1 \mathrm{~cm}$ so that pions (mainly produced transversely) escape with only a small chance of being absorbed. Beryllium was chosen as the target material because for several reasons: it has a high pion production yield, minimizes remnant radioactivity from the large proton exposure, and resists material fatigue from the high beam rate. The target is air cooled to dissipate the $\sim 600 \mathrm{~W}$ of power deposited by both primary and secondary beam particles 1 . The target is surrounded by the horn, though the two are structurally separate so that the target can be removed in case of a problem. However, electrical contact is maintained between the two pieces to prevent arcing due the electrical currents in the horn.

These large currents generate the strong magnetic fields that focus the secondary beam. Current travels along the horn's inner conductor and back out along its outer conductor in a train of ten $143 \mu \mathrm{s}$ long pulses separated by $1 / 15 \mathrm{~s}$. The horn then cools for $\sim 1.5 \mathrm{~s}$ until the next ten-pulse train. The direction of current can be reversed, changing the sign of the magnetic field and producing a $\bar{\nu}_{\mu}$ beam. The horn pulse duration is designed so that the shorter $16 \mu$ s proton pulse from the Booster intercepts the horn at maximum current: $170 \mathrm{kA}$.

\footnotetext{
${ }^{1}$ Flowing air at $8 \mathrm{l} / \mathrm{s}$ along the target keeps it at $120^{\circ} \mathrm{C}$.
} 


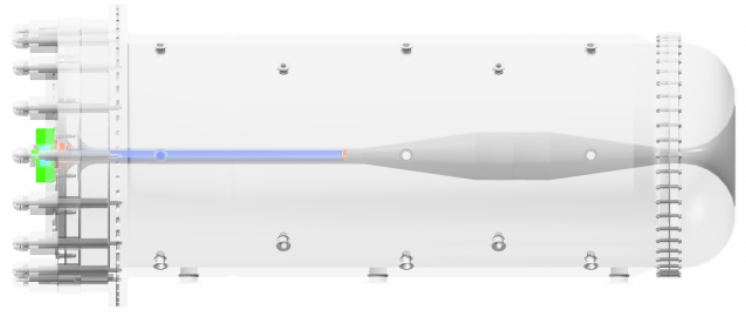

Figure 3.3: The horn assembly. The outer conductor is rendered transparent to show the shape of the inner conductor [76].

The magnetic field produced at this peak current is $\sim 1 \mathrm{~T}$ between the inner and outer conductors (it is negligible elsewhere). Before horn installation, the field was measured to be the same as the field from an ideal line current: $B=\mu_{0} I / 2 \pi r$ to a $10 \%$ uncertainty $(r$ is the distance away from the inner conductor) [74].

The magnitude of the magnetic field and the shape of the inner conductor were optimized in a full GEANT [75] simulation to maximize the $\nu_{\mu}$ flux between 0.5 and $1.0 \mathrm{GeV}$, but minimize it at higher neutrino energies. The inner conductor radius varies from $2.2 \mathrm{~cm}$ to $6.54 \mathrm{~cm}$, whereas the outer conductor radius is a constant $30 \mathrm{~cm}$ (figure 3.3). The entire horn (184.5 cm long) is enclosed in an airtight box (coffin) designed to contain radioactive air for a minimum of four hours.

Running the horn produces a large heatload that is carried off by water sprayed onto the inner conductor. Water from a closed-circuit radioactive water (RAW) system flowing at $1 \mathrm{l} / \mathrm{s}$ removes power at a rate of $3000 \mathrm{~W} / \mathrm{m}^{2} / \mathrm{K}$. The water is sprayed across the length of the inner conductor by eighteen vibration-isolated nozzles attached to the outer conductor 


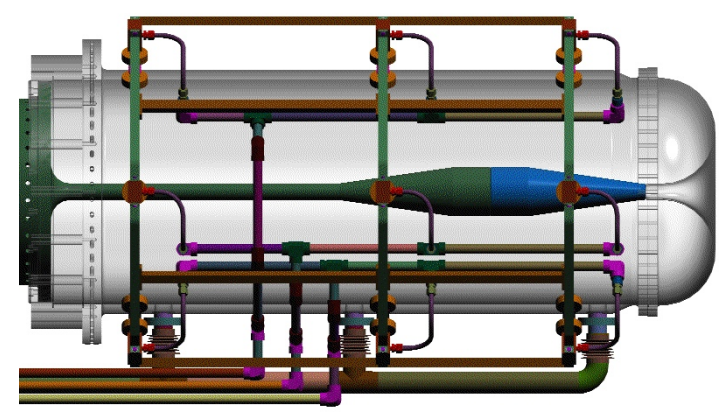

Figure 3.4: The horn assembly with external cooling water system (truss). The outer conductor is rendered transparent [76].

with stainless steel bellows. The piping for the RAW system is supported by a truss that surrounds but does not touch the horn (figure 3.4). This isolation helps prevent fatigue failure of the piping that might otherwise be caused by the mechanical shock of pulsing the horn.

The MiniBooNE horn design has been a successful one. Comparisons of horn-on and horn-off data show that it increases the $\nu_{\mu}$ flux by a factor of $\sim 5$ [77]. It has a large momentum and angular acceptance of produced pions $\left(1<p_{\pi}<4 \mathrm{GeV} / \mathrm{c}\right.$ and $0<\theta_{\pi}<$ 0.2 rads). It is also designed to have a long lifetime: 200 million pulses. The first horn pulsed 96 million times before a ground fault made it unsafe to operate. Despite this, when it was decomissioned, it held the record for the most pulses on a single horn. The second horn has pulsed 240 million times and is still in use by the experiment.

\subsubsection{Tertiary (Neutrino) Beam}

The beam that exits the horn consists mainly of scattered and unscattered primary protons and secondary mesons. A steel collimator $\sim 2$ m downstream of the horn stops hadrons $(\pi$, 
$K, p, n)$ that would deposit their energy in the enclosure walls instead of entering the decay region. Forward-going mesons $(\pi$ and $K$ ) enter this region and decay in flight to produce the tertiary neutrino beam.

The decay region is a $50 \mathrm{~m}$ long air filled cylinder (pipe) with $90 \mathrm{~cm}$ radius. At the end of the region is a permanent $\sim 300$ tons steel absorber to stop the hadronic and muonic portions of the beam. A second absorber can be lowered into the decay pipe $\sim 25$ m downstream of the target allowing for systematic studies of the neutrino flux.

The majority of this flux comes from pion and kaon decay: $\pi^{+} \rightarrow \mu^{+} \nu_{\mu}$ and $K^{+} \rightarrow \mu^{+} \nu_{\mu}$. Kaons can also decay to produce electron neutrinos which are an "intrinsic" background to the oscillation search. Decay of muons $\mu^{+} \rightarrow e^{+} \bar{\nu}_{\mu} \nu_{e}$ contribute to this background as well. However, the proportion of intrinsic $\nu_{e}$ 's is small and can be characterized experimentally. Both the $\nu_{e}$ from $\pi$ and $K$ can be constrained from measurements of the corresponding $\nu_{\mu}$ events in the detector $\left(\nu_{\mu}\right.$ from $K$ tend to be produced at higher energies than those from $\pi)$. Additional information on intrinsic $\nu_{e}$ from $K$ decay is obtained via a detector in the beamline called the Little Muon Counter (LMC) which directly measures the $\mu$ from $K$ decay $[78$.

Only neutrinos successfully transit the permanent absorber at the end of the decay region. They then travel through $\sim 500 \mathrm{~m}$ of dirt before intercepting the detector.

\subsection{Detector}

The MiniBooNE detector (figure 3.5) is a $610 \mathrm{~cm}$ radius steel sphere situated underground in a concrete vault $\sim 550 \mathrm{~m}$ downstream of the target. It is instrumented with over 1500 photomultiplier tubes (PMTs), and filled with $\sim 1 \times 10^{6} 1$ of mineral oil $\left(\mathrm{CH}_{2}\right)$ which makes up $\sim 95 \%$ of the detector mass. The detector volume is divided into two optically isolated regions separated by a phototube support structure at a radius of $575 \mathrm{~cm}$. The inner volume 


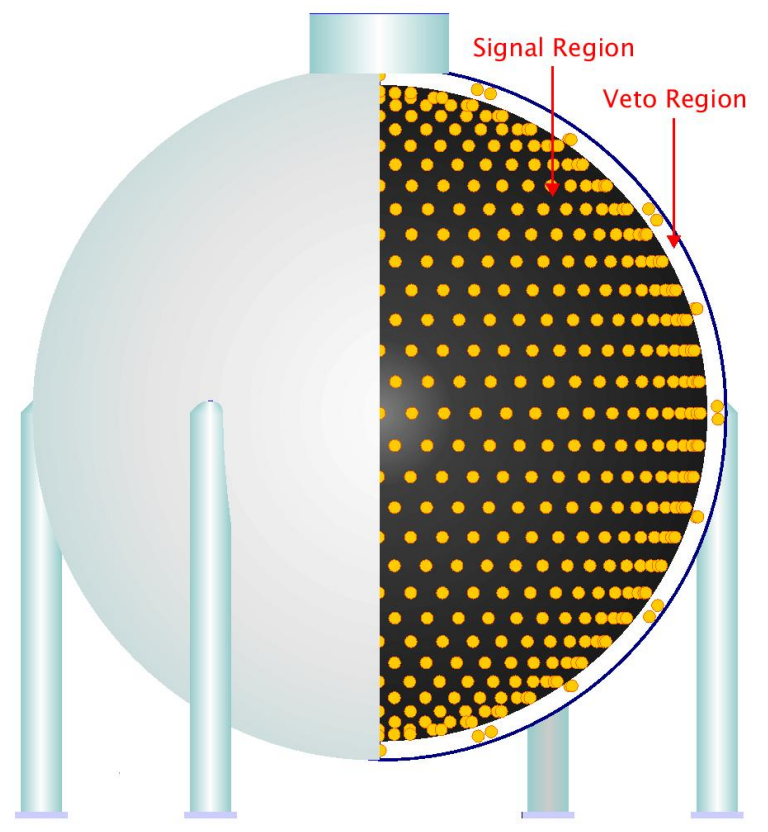

Figure 3.5: Cut-away of the MiniBooNE detector showing the main signal volume and outer veto region.

is the main detector region, while the outer volume serves to veto charged particles entering and/or exiting the detector (generally cosmic rays). This thin shell (35 cm thickness) is enough to satisfactorily veto cosmic rays because the detector is shielded from above by an overburden of dirt. The overburden provides a mimumum barrier of $3 \mathrm{~m}$ to cosmic ray particles, limiting their rate in the detector to $<10 \mathrm{kHz}$.

The phototube support structure undergirds 8 inch diameter Hamamatsu [80] PMTs that observe light from from neutrino interactions in the detector. There are 1280 PMTs attached via the wire frame shown in figure 3.6 to the inner surface of the barrier to monitor the main detector region. These provide $\sim 10 \%$ photocathode coverage of that surface. Of these, 950 are 9-stage Hamamatsu model R1408 tubes recycled from LSND. The remainder are 10-stage model R5912 (an upgrade to the LSND tubes) purchased specifically for 


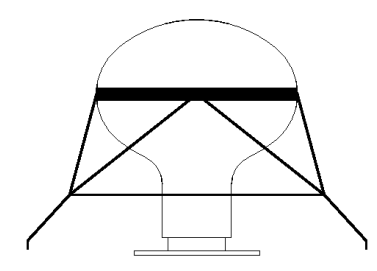

Figure 3.6: MiniBooNE PMT in wire support-frame used to attach PMTs to the optical barrier [79].

MiniBooNE. A further 240 tubes instrument the veto region all of which are the LSND model.

Each phototube was tested prior to installation to characterize its time and charge response and to determine its proper operating voltage [81]. Every tube is set at its own voltage to provide a uniform gain of $\sim 10^{7}$. Other properties of the tubes such as dark current, jitter, charge resolution, double pulsing, and pulse shape were also measured. Table 3.1 includes some relevant numbers.

\begin{tabular}{ll}
\hline \hline charge resolution & $\sim 15 \%$ @ 1 p.e. \\
time resolution & $\sim 1 \mathrm{~ns}$ \\
average PMT voltage & $1800 \mathrm{~V}$ \\
avgerage dark rate & $3 \mathrm{kHz}$ \\
wavelength range & $300-650 \mathrm{~nm}$ \\
\hline
\end{tabular}

Table 3.1: Selected photomultiplier tube properties.

Of the old tubes, those with the best resolution were installed in the main detector region, while the others went to the veto region where the reconstruction requirements are less stringent. In the veto, it is important simply to detect as much light as possible. To facilitate this the inner surface of the detector and the outer surface of the barrier are 


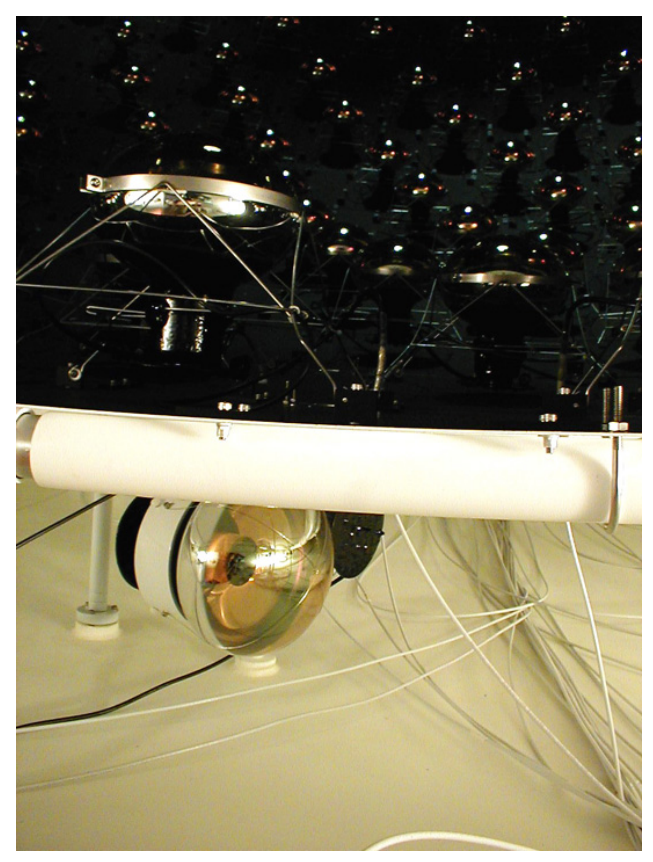

Figure 3.7: Photograph of the main signal (top) and veto (bottom) regions with attached PMTs.

painted white to maximize reflections, giving the best chance of detecting photons. The reflectivity of surfaces in the veto region has been measured to be larger than $80 \%$. In the main detector region, it is important to minimize reflections since they would interfere with particle indentification and event reconstruction. So the inner surface of the barrier is painted a flat black. Figure 3.7 shows a photograph of the two regions.

For optimal transmission of light to the phototubes, the oil must posses certain properties. These include photon emission in the usable range of the PMTs, low attenuation, low dispersion in the transmission range, high refractive index, low reactivity with detector materials, and a low enough viscosity to allow the oil to flow. Some relevant numbers, including the expansion coefficient, are given in table 3.2 .

Oil expansion due to temperature changes is a serious issue for MiniBooNE due to its 


\begin{tabular}{ll}
\hline \hline density & $0.845 \pm 0.001 \mathrm{~g} / \mathrm{cm}^{3}$ \\
refractive index & $1.4684 \pm 0.0002\left(@ 589.3 \mathrm{~nm}, 20.0^{\circ} \mathrm{C}\right)$ \\
attenuation length & $14 \pm 2 \mathrm{~m}(@ 400 \mathrm{~nm})$ \\
expansion coefficient & $0.1 \%$ per $1^{\circ} \mathrm{C}$ \\
\hline
\end{tabular}

Table 3.2: Selected measurements of oil properties.

large size. A temperature change of $1^{\circ} \mathrm{C}$ equals a volume change of 1000 liters. The impact of this is amplified by the Erlenmeyer flask shape of the detector tank: a cylindrical access portal tops the main detector sphere. This shape transforms a change of $1^{\circ} \mathrm{C}$ to a change of 10 inches of oil level in the portal.

A 1000 liter buffer tank connected to the detector mediates these issues. If the oil level rises too much, a pipe at the top of the access portal drains oil to the buffer tank. If the level drops too much, oil can be pumped from the buffer tank through the detector fill line to the bottom of the detector. Figure 3.8 shows the relative location of the main and buffer tanks. In practice, the oil in the detector is kept below the ambient vault temperature and allowed to rise at a very slow rate. This ensures that the oil level is always as high as the drain pipe.

This plumbing system also allows oil to be circulated between the two tanks. MiniBooNE utilized this ability after the initial fill to saturate the oil with nitrogen by bubbling it into the buffer tank while circulating the oil. Nitrogen displaces oxygen which can degrade the oil affecting light progation [82]. Nitrogen levels in the detector tank are preserved postsaturation by bubbling small quantities of gas directly into the detector, maintaining an overpressure of nitrogen in the access portal. Appendix A provides further details.

The access portal also has penetrations for PMT signal cables that connect the photomultipliers to the front end electronics. The top of the portal is located in the detector 


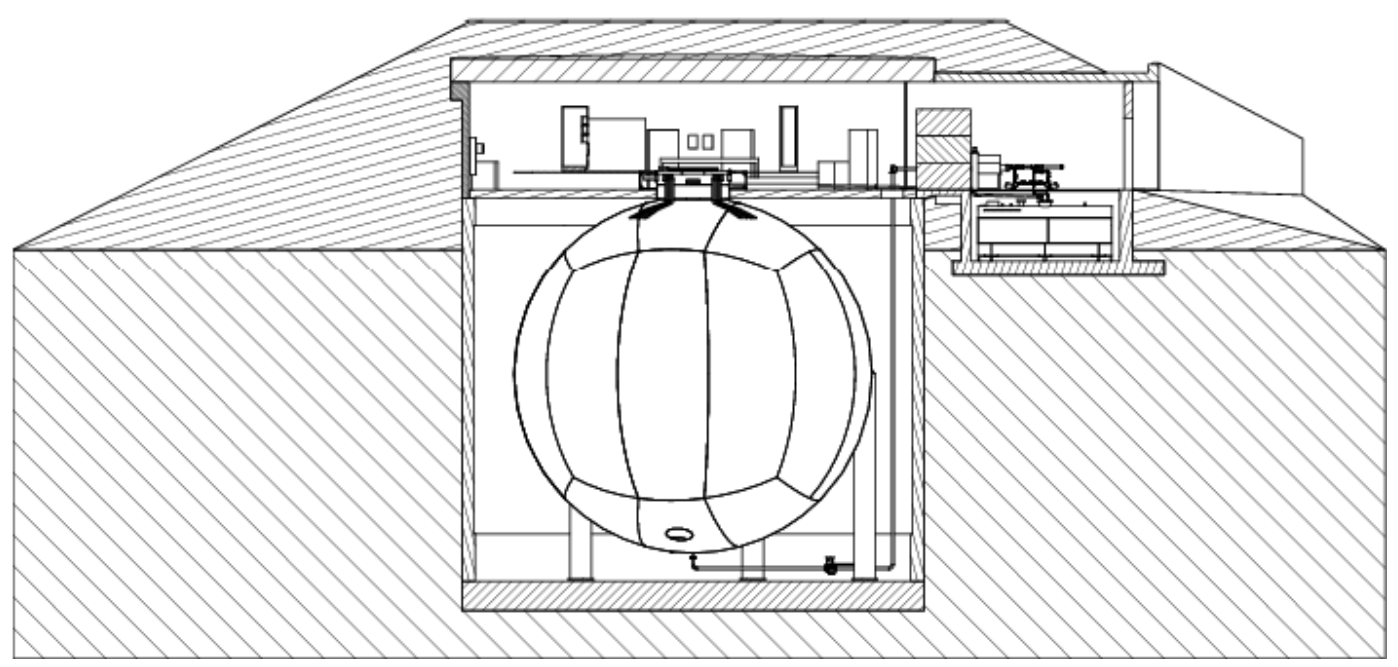

Figure 3.8: Elevation showing the MiniBooNE detector and buffer tanks as well as the support building and dirt overburden [79].

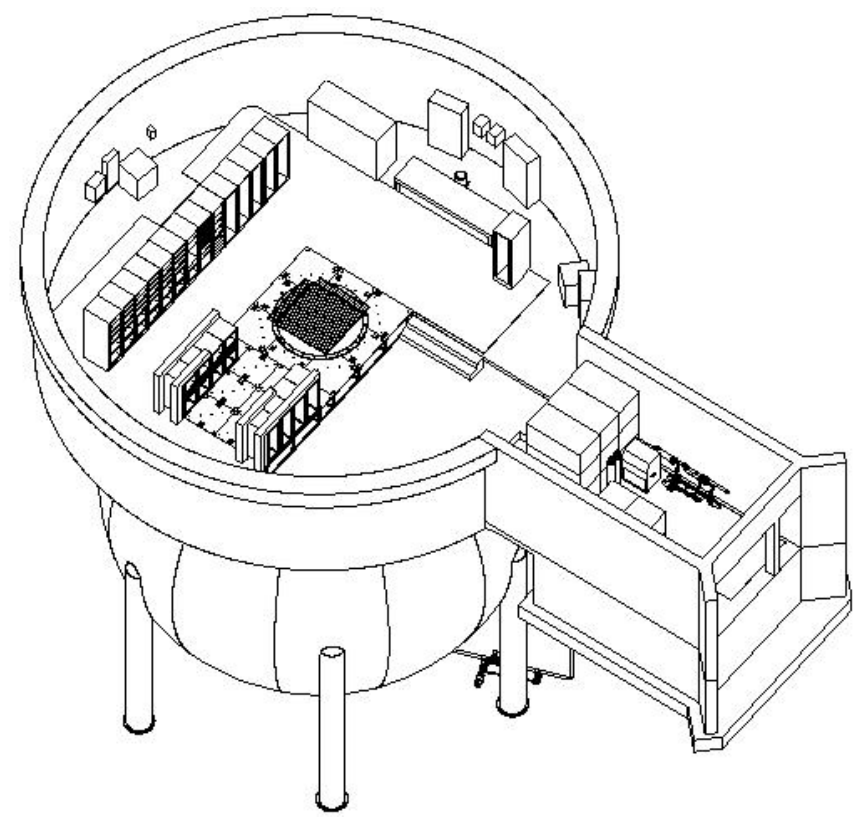

Figure 3.9: Perspective drawing of MiniBooNE with emphasis on the support plant [79]. 
support plant (figure 3.9), the building above the vault that houses the front-end electronics for the PMTs, the data acquisition electronics, certain calibration and monitoring systems, as well as utilities (power and HVAC).

\subsection{Data Acquisition}

The electronics and data acquisition systems housed in the support building convert analog photomultiplier tube signals into digital charge and time information for each PMT and build that information into events. Instead of digitizing the full signal waveform for each of the 1520 PMT channels, MiniBooNE uses fast analog filters to transform the signal pulse into modified charge and time waveforms. These modified voltages are digitized at $100 \mathrm{~ns}$ intervals called "clock ticks".

Figure 3.10 represents the digitization of one channel. The top trace shows the PMT signal pulse from which the integrated charge $(\mathrm{Vq})$ trace is generated. When the PMT signal voltage crosses a threshold equivalent to $\sim 0.25$ photoelectrons, a discriminator starts the integrated time ramp (Vt). The digitized information from this voltage provides a sub-nanosecond precision measurement of the signal time.

This charge and time data are stored in $204.8 \mu$ s circular buffers until the trigger decides whether to keep the event. When triggered, the data in the buffers are transferred to the main acquisition computer where events are assembled and stored. The following information is kept: the clock tick number just before the timing pulse fires $(t-1)$, and the four digitized Vq and Vt values from clock ticks $\mathrm{t}-1$ through $\mathrm{t}+2$ called a "quad". If the trigger takes longer than the $204.8 \mu$ s buffer time to make a decision, that data is overwritten, and the event is lost. Such an event is called "latent", and is removed from analysis by downstream software filters (see Chapter 5). Only a few tenths of a percent of beam events are latent. 


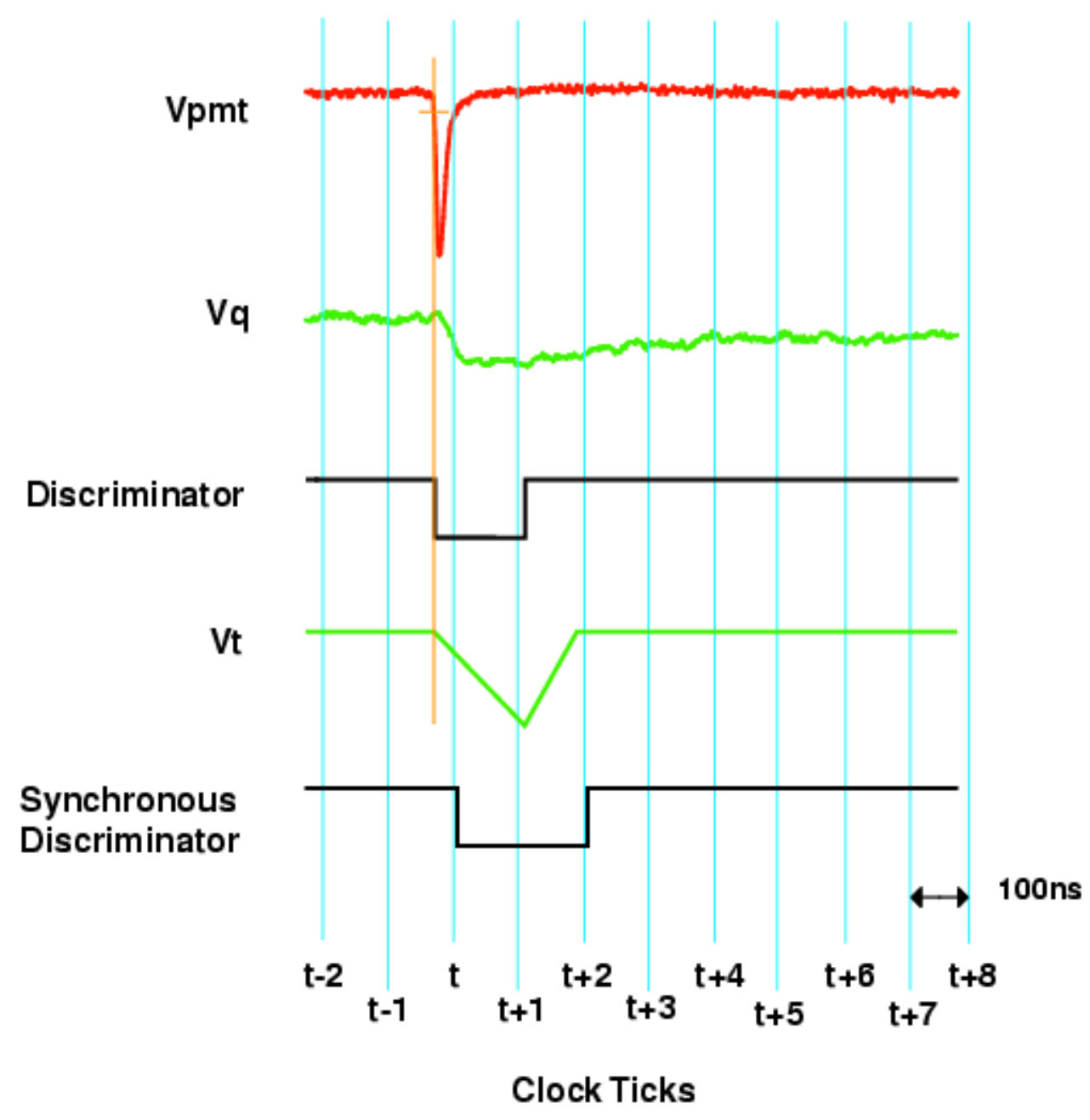

Figure 3.10: DAQ signal traces. Vq and Vt traces are derived from the upper Vpmt trace and digitized at 100ns intervals (clock ticks) [79]. 
A variety of triggers are implemented in the DAQ to determine whether an event should be stored [83]. However, only the two used for the neutral current elastic analysis will be described here. The most important of these is the beam trigger which takes precedence over (inhibits) all other triggers. It is the basis for all neutrino induced events described in later chapters. The beam trigger is set when the DAQ receives timing signals from the Booster indicating that protons are being extracted to MiniBooNE. It opens a $19.2 \mu$ s long time window that surrounds the $1.6 \mu$ s long beam spill. The next most important trigger for this analysis is the "strobe" trigger. This is also $19.2 \mu \mathrm{s}$ long and is set by a pulse generator operating at $2.01 \mathrm{~Hz}$. This trigger provides a random sample of beam-unrelated activity in the detector.

\subsection{Calibration Systems}

The raw information collected by the electronics and stored by the DAQ for each phototube must be calibrated to be useful for analysis. This calibration determines the true time and actual charge collected by each PMT. The detector as a whole must also be calibrated to determine its energy range and resolution.

\subsubsection{PMT Response \& Calibration}

The PMT charge and time "quad" recorded by the DAQ becomes useful for event reconstruction and particle ID only after conversion to an absolute measurement of charge in photoelectrons and time in nanoseconds. To perform such a calibration, it is necessary to quantify the charge and time responses of each PMT. MiniBooNE accomplishes this with a laser system designed to calibrate the PMTs in situ over the lifetime of the experiment.

A pulsed diode laser external to the detector connects to five optical fibers via a switch box. Four of these fibers terminate in the detector in flasks filled with a dispersive medium 
called Ludox ${ }^{\circledR}$ that allows essentially isotropic reemission of light into the oil. Each of these flasks is located at different positions in the detector (table 3.3 ). The fifth fiber is bare, and light exits that fiber in a $10^{\circ}$ cone that illuminates a small circle of phototubes at the bottom of the detector. Both the intensity of the laser and frequency of the pulses are adjustable allowing a variety of calibration studies. In general the laser operates at $\sim 80 \%$ intensity and pulses at $\sim 3.3 \mathrm{~Hz}$ asynchronously with proton delivery to MiniBooNE.

\begin{tabular}{lcccc}
\hline Device & radius $(\mathbf{c m})$ & $\mathbf{x}(\mathbf{c m})$ & $\mathbf{y}(\mathbf{c m})$ & $\mathbf{z}(\mathbf{c m})$ \\
\hline Flask \#1 & 4.4 & -0.3 & -4.1 & 1.5 \\
Flask \#2 & 215.0 & 144.9 & 96.1 & -126.4 \\
Flask \#3 & 83.7 & 1.7 & -0.8 & 83.7 \\
Flask \#4 & 220.3 & -80.0 & 203.9 & -24.1 \\
Bare Fiber & 550.0 & 82.0 & 540.0 & 65.0 \\
\hline
\end{tabular}

Table 3.3: Locations of laser flasks and the termination point of the bare fiber in beam coordinates ( $\mathrm{z}$ along beam, $\mathrm{y}$ is up, and $\tan \phi=z / x$ ).

The nature of PMT calibrations performed using the laser system is determined by the internal workings of the phototubes (figure 3.11). Light incident on the photocathode ejects electrons via the photoelectric effect. These photoelectrons are accelerated by an electric field to the first dynode (figure 3.12). Here the energy of impact ejects (in general) a larger number of secondary electrons that are then accelerated to the next dynode. This process continues through the dynode chain until enough electrons to form a measurable current are collected by the anode.

The process of converting photons to a measured current is statistical at each stage and so subject to variability. This is most apparent in the quantum efficiency of the tubes. Not every photon generates a measured current, an effect that depends on the wavelength of the incident photon. Figure 3.13 shows the quantum efficiency for new (R5912) PMTs 85]. 


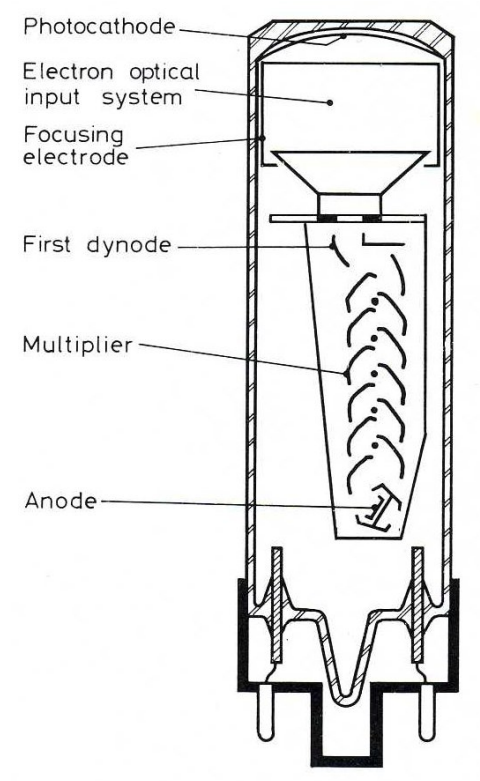

Figure 3.11: Cut away of a PMT (different photocathode shape than used in MiniBooNE) [84.

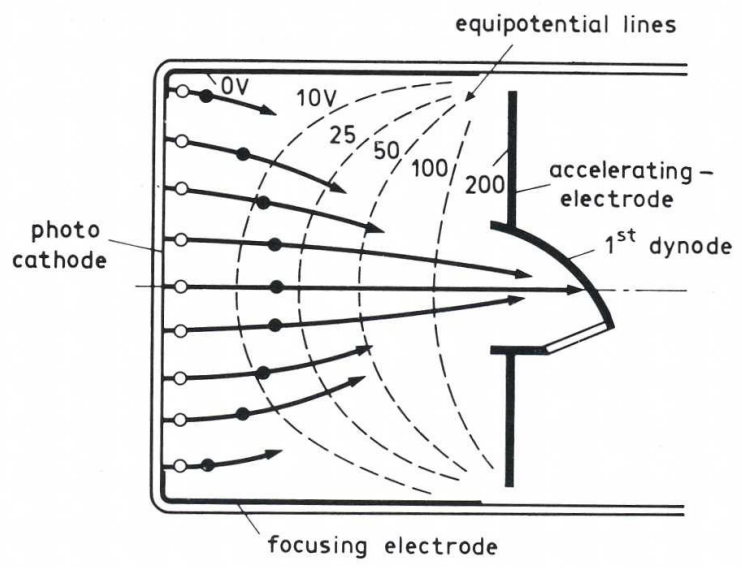

Figure 3.12: Schematic of photoelectron paths from different parts of the photocathode to the first dynode 84]. 


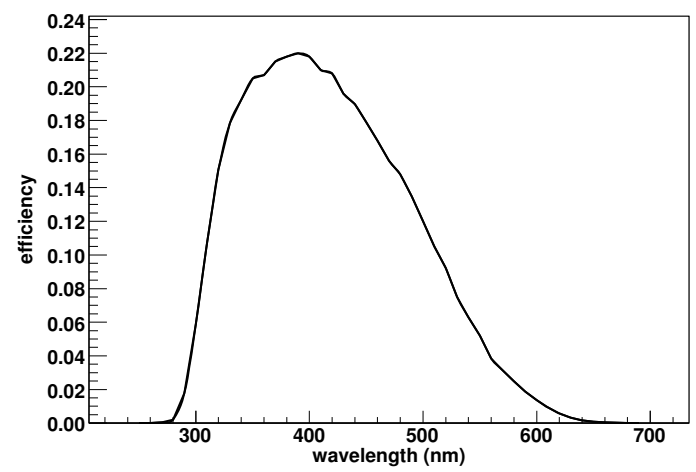

Figure 3.13: Photocathode quantum efficiencies as a function of wavelength for R5912 phototubes.

The statistical nature of the phototubes also causes fluctuations in the gain, the overall factor by which the the number of electrons increases from the photocathode to the anode $\left(\sim 10^{7}\right.$ in MiniBooNE). Variations in the gain are variations in the raw measured charge (from the DAQ quad) for a given number of initial photoelectrons. MiniBooNE measures the distributions of these fluctuations for each PMT by pulsing low intensity laser light into one of the flasks (generally the central flask). The light level is tuned such that PMTs only observe a single photoelectron.

The distribution of single photoelectron events in a PMT is the charge response for that tube: how much raw charge is measured per photoelectron. The mean of this $\left\langle Q_{\text {raw }}^{1 P E}\right\rangle$ is used to calibrate the measured raw charge of a PMT:

$$
q=\frac{Q_{\text {raw }}}{\left\langle Q_{\text {raw }}^{1 P E}\right\rangle}
$$

where $q$ is the calibrated charge in photoelectrons and $Q_{\text {raw }}$ is the charge measured by the DAQ for a particular event [86]. The width of this single photoelectron distribution gives the charge resolution. The average charge resolution for MiniBooNE tubes is $\sim 15 \%$ for 1 photoelectron. 
As with charge response, there is also variability in time response. This can be caused by the initial position, direction, and energy of the emitted photoelectrons as well as by the number emitted. Signals from events with more photoelectrons tend to be collected sooner than those with fewer, a process known as time slewing. Just as with charge, the time calibrations for each PMT can be extracted from laser events, though both single and multi-photoelectron event distributions are collected. The calibrated time is given by

$$
t=T_{\text {raw }}+\Delta t_{\text {offset }}+\Delta t_{\text {slew }}(q)
$$

where $T_{\text {raw }}$ is the raw time information determined from the DAQ quad, $\Delta t_{\text {offset }}$ is a PMTdependent constant that accounts for timing differences from things such as cable lengths and dynode structure, and $\Delta t_{\text {slew }}(q)$ is the correction for time slewing. Time resolutions in MiniBooNE are $~ 1.6 \mathrm{~ns}$ for R1408 tubes and $1.2 \mathrm{~ns}$ for R5912 tubes.

The calibrated time distribution using the laser system on R1408 (old) phototubes is shown in figure 3.14. In general, the desired signal is the "prompt light" peak that dominates the distribution (note the $\log \mathrm{y}$-scale). The rest of the structure comes from timing features common to phototubes. "Dark noise" typically comes from thermionic emission of electrons from the cathode and dynodes. "Pre-pulsing" occurs if the photon passes through the photocathode and strikes the first dynode directly. This causes the hit to be reconstructed early. "Late-pulsing" can be caused by ionization of remnant gas in the phototube. "Reflections" come from the detector wall and phototube faces. "Scattering" is a property of the oil that affects the detector response, and is discussed further in section 3.6 .

\subsubsection{Detector Calibration}

It is as important to calibrate the whole detector as it is each individual phototube. MiniBooNE uses a cosmic muon calibration system to precisely measure the energy, position, and direction of muons in the full range of interest to the experiment. The system also 


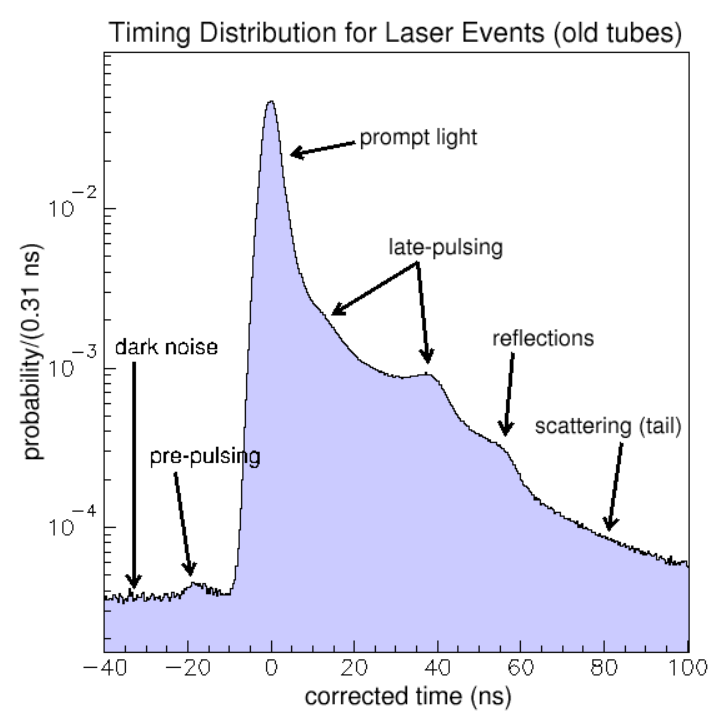

Figure 3.14: Calibrated (labelled "corrected") time distribution of R1408 PMTs for laser light from flasks.

calibrates electrons with energy less than $50 \mathrm{MeV}$ using electrons produced by muons that decay at rest.

The cosmic muon calibration system consists of a muon tracker above the detector in the support building, and scintillator cubes in the detector fiducial volume. The muon tracker is two sets of two layers of scintillator hodoscope separated by $\sim 1 \mathrm{~m}$ that determines the position and direction of muons entering the detector. On its own, the tracker provides a high statistics sample of muons that calibrate direction reconstruction to an angular resolution of $\sim 3^{\circ}[87,88$.

The scintillator cubes are permanently located at different depths in the detector between 15 and $400 \mathrm{~cm}$ below the muon tracker. Each is $5 \mathrm{~cm}$ on a side, optically isolated from the detector oil, and connected via optical fiber to a 1 inch PMT outside the detector. The cubes give the terminal position of the $\sim 500$ cleanly measured muons per month that 


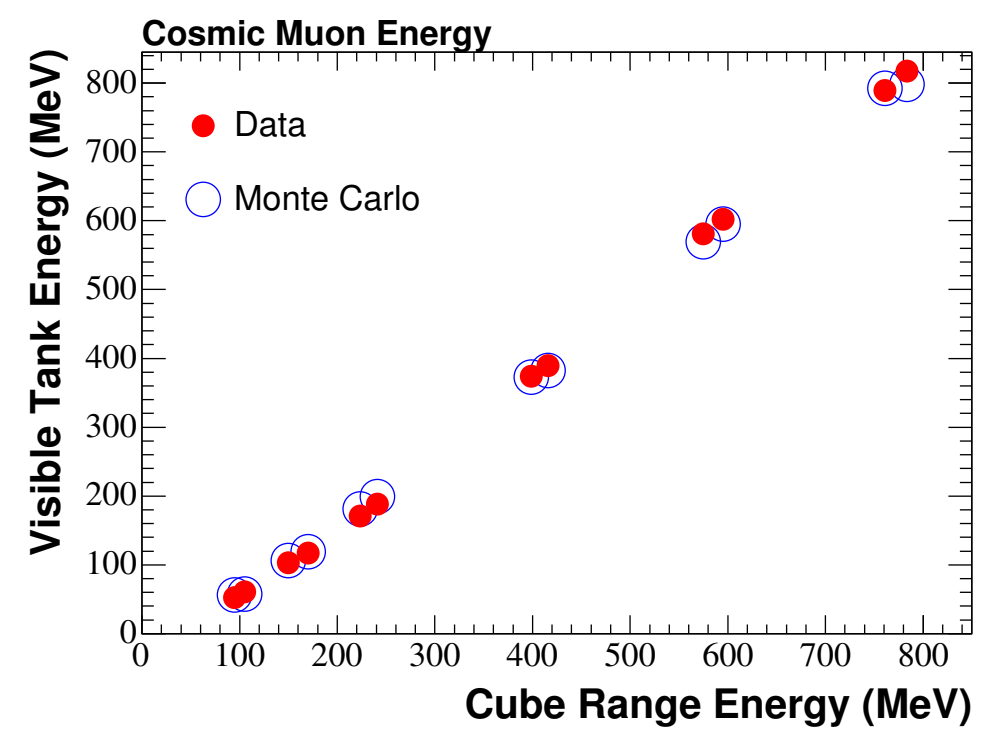

Figure 3.15: Cosmic muon energy calibration shown with predictions from a Monte Carlo simulation (discussed in Chapter 4). The visible energy is an "electron equivalent" energy which explains deviations from a straight line.

stop in them with an uncertaintly less than $3 \mathrm{~cm}$.

The measurement of range from the muon tracker and cubes provides muon energy resolution to a $\sim 3 \%$ uncertainty that is almost entirely due to fluctuations in muon energy loss. This sets the absolute energy scale calibration for muons between 20 and $800 \mathrm{MeV}$ to $\pm 1 \%$ [89]. Figure 3.15 shows how the energy determined from muon range calibrates the visible energy. This is also used to tune the light propagation model used in the simulations (discussed in Chapter 4).

When a muon stops, it decays into a "Michel" electron. The energy distribution of Michel electrons is well known and can be used to determine both the energy resolution and scale for low energy electrons. MiniBooNE has collected large samples of Michel electrons both from stopping cosmic muons and muons produced via neutrino interactions. The 


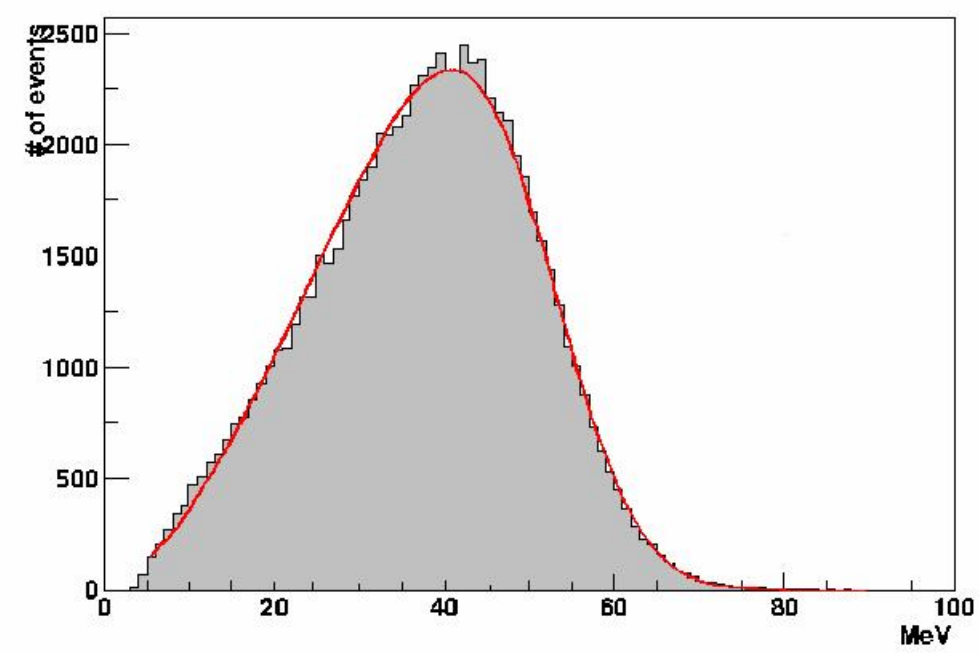

Figure 3.16: Observed Michel electron energy distribution (histogram) compared with the best fit (solid line) convolution of the theoretical energy distribution and a gaussian with width proportional to $\sqrt{E}$. The energy resolution is $15 \%$ at $50 \mathrm{MeV}$.

measured energy resolution for these particles is $\sim 15 \%$ at $53 \mathrm{MeV}$ (figure 3.16).

\subsection{Light Emission and Propagation}

In general light generated from a neutrino interaction in MiniBooNE must travel through meters of oil before being detected by a phototube. To understand this process, it is useful to consider both the emission and transmission of this light.

\subsubsection{Light Emission}

In MiniBooNE, light is emitted by charged particles via two processes: Cherenkov and scintillation radiation. 


\section{Cherenkov Radiation}

Cherenkov radiation is the coherent emission of light by a charged particle moving through an insulating medium at superluminal velocities. The velocity of light in a medium $c_{n}$ is characterized by the index of refraction $n$ of that medium: $c_{n}=c / n$. Charged particles that move faster than $c_{n}$ (the Cherenkov threshold) create a photonic shockwave analogous to the sonic boom caused by supersonic particles. The wavefront of this shock is conical and emitted with a characteristic opening angle $\theta_{C}$ defined by

$$
\cos \theta_{C}=\frac{1}{\beta n(\lambda)}
$$

where $\beta$ is the relativistic velocity $v / c$ and $n(\lambda)$ shows the explicit dependence of the refractive index on the wavelength of the emitted light $\lambda \bigsqcup^{2}$

The conical shockwave travels as shown in figure 3.17 with $\theta_{C}$ decreasing as the particle's velocity decreases until it finally drops below Cherenkov threshold. The disturbance is detected as a ring (the cross section of a cone) on the detector wall. For a particle of charge ze moving above threshold, the number of photons emitted per unit wavelength per unit path length is

$$
\frac{d^{2} N}{d \lambda d x}=\frac{2 \pi \alpha z^{2}}{\lambda^{2}} \sin ^{2} \theta_{C}
$$

where $\alpha$ is the fine structure constant $\left(e^{2} / \hbar c\right)$ [84]. This light is prompt: emitted immediately upon passage of the particle.

\section{Scintillation Radiation}

The other mechanism by which charged particles emit light in the detector is scintillation. Scintillation is a by-product of ionization energy loss and occurs when charged particles

\footnotetext{
${ }^{2}$ In MiniBooNE, $n(\lambda)$ is characterized by the Cauchy parameterization $n(\lambda)=n_{D}+B\left(\frac{1}{\lambda}+\frac{1}{\lambda_{D}}\right)$ where $\lambda_{D}=589.3 \mathrm{~nm}$ is the sodium doublet Fraunhofer D line, and the parameters $n_{D}=n\left(\lambda_{D}\right)=1.4684 \pm 0.0002$ and $B=4240 \pm 157 \mathrm{~nm}^{2}$ have been measured at $20^{\circ} \mathrm{C}$ for MiniBooNE oil 90].
} 


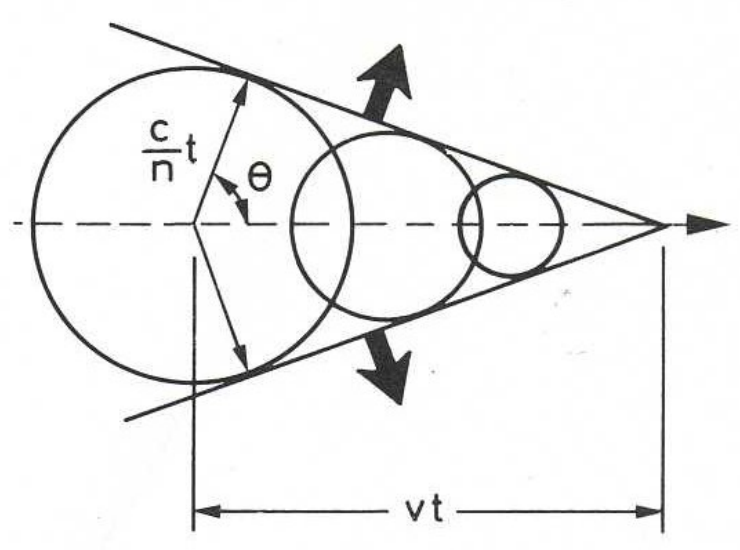

Figure 3.17: Cherenkov radiation schematic [84].

travelling through the mineral oil excite electron states of organic molocules in their path. The resulting de-excitation of these states emits light that is isotropic and delayed with respect to the passage of the particle. The emission wavelengths and time spectra of this light are dependent on the chemical composition and molecular structure of the oil. Neutrons can also produce scintillation light, though indirectly by producing protons via scattering or charge exchange.

Scintillation is only approximately proportional to the ionization loss of particles in the the oil. In general effects between excited molecules such as recombination and quenching reduce the light yield [91]. The corrected energy loss is described by Birk's saturation law in terms of the ionization energy loss:

$$
\frac{d E^{\prime}}{d x}=\frac{d E / d x}{1+k_{B} d E / d x}
$$

where $k_{B}=0.014 \mathrm{~g} / \mathrm{cm}^{2} / \mathrm{MeV}$ is Birk's constant for mineral oil.

Measurements to characterize scintillation production in MiniBooNE mineral oil were carried out using the Indiana University Cyclotron Facility (IUCF) $200 \mathrm{MeV}$ proton beam. Results from these studies showed scintillation light with a characteristic time of $\sim 19 \mathrm{~ns}$ 


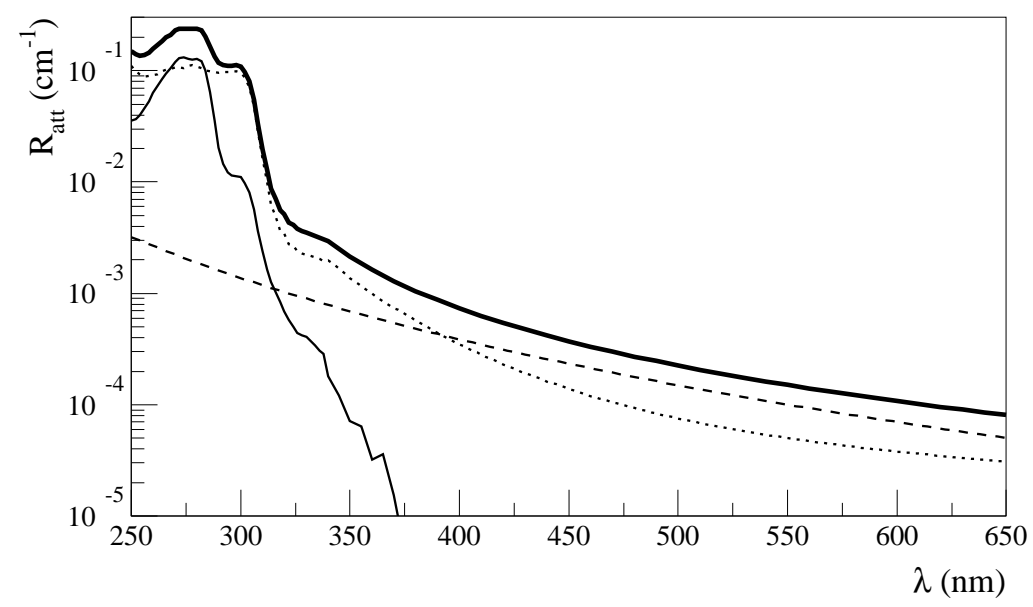

Figure 3.18: Attenuation rate in MiniBooNE mineral oil as a function of wavelength. The thick solid curve is the total rate, the thin solid curve comes from fluorescence, the dashed curve from scattering, and the dotted curve from absorption [94].

[92].

\subsubsection{Light Transmission}

Once emitted, the photons must travel through the oil to the phototubes where they can be measured. However there are processes along the way that attenuate (reduce the intensity along the original direction of) the emitted light. In MiniBooNE these processes are fluorescence, scattering, and absorption.

Measurements of fluorescence and scattering rates have been made for the mineral oil, as has the total attenuation rate [93]. The absorption rate is not measured but defined to be the difference between the attenuation rate and the fluorescence and scattering rates. A summary of the attenuation measurements and its components is shown in figure 3.18 . 


\section{Fluorescence}

Fluorescence occurs when an optical photon from either Cherenkov or scintillation processes excites electrons in an organic molecule called a "fluor". The electrons de-excite to an interim state emitting a lower energy (longer wavelength) photon: a process called a Stokes transition. The photon is emitted isotropically and with a characteristic time less than $10^{-7} \mathrm{~s}$.

Mineral oil contains both natural and manufacturer-added fluors (e.g. Vitamin E which is added as an antioxidant and a stabilizer). Extensive measurements have been used to completely characterize four fluors that are of relevance to MiniBooNE [95, 96].

\section{Scattering}

Two other processes that attenuate light during transmission are Raman and Rayleigh scattering. Raman scattering is similar to fluorescence in that an incident photon excites an electron which then makes a Stokes transition emitting a less energetic photon. However here, the energy difference is converted into rotational or vibrational modes in the molocule.

Rayleigh scattering occurs when a photon excites an electron which de-excites by returning to its original state emitting a photon of the same frequency (but now in a new direction). This is the most common process by which quasimonochromatic light is scattered in a substance [97]. Tests performed at Fermilab confirmed this showing that $\sim 95 \%$ of scattered light comes from Rayleigh scattering while only $~ 5 \%$ comes from Raman scattering [98.

The information presented regarding scattering, the other optical properties of the oil, and indeed every part of the experimental setup from the initial $8 \mathrm{GeV}$ protons is modelled by Monte Carlo event simulations discussed in the next Chapter. 


\section{Chapter 4}

\section{Event Simulation and}

\section{Reconstruction}

Each stage of the experiment described in the previous chapter must be simulated from initial protons to data storage. These simulated events as well as the real data are then reconstructed from simple charge and time information to more physically meaningful variables (such as energy or number of emitted scintillation photons) which provide the basis of analysis. This chapter describes the simulation and reconstruction processes and routines.

\subsection{Beam and Flux Simulations}

The MiniBooNE beam and flux simulations are coordinated by three separate programs. The first, a GEANT4-based Monte Carlo [75], simulates the MiniBooNE beam starting from primary protons interacting with the beryllium target and ending at the decays of the secondary particles (mesons and muons) that yield neutrinos. The second, a FORTRAN based program, re-decays each neutrino parent 1000 times so that the beam Monte Carlo incurs negligible statistical errors. The final program, also FORTRAN based, uses the re- 
decayed events to determine the neutrino energy spectrum for each neutrino type as input to the cross section Monte Carlo discussed in the next section.

The GEANT4 program generates the initial protons at the target according to the actual MiniBooNE beamline optics. It models the geometry and materials of all objects in the target hall and decay region as well as the horn's magnetic field. The Monte Carlo then tracks the particles involved in neutrino production through these materials and the magnetic field, accurately simulating the physics processes that govern the interactions of these particles.

The most important of these interactions for neutrino flux predictions are the inelastic interactions of primary protons with the beryllium target since it is the secondary beam particles produced in these interactions $\left(\pi^{ \pm}, K^{ \pm, 0}\right.$, protons, and neutrons) that yield the neutrinos. MiniBooNE employs three methods to model the production cross sections for these interactions. A Sanford-Wang parameterization describes the double-differential inclusive production cross sections for $\pi^{ \pm}$and $K^{0}$; production of $K^{+}$is handled with a Feynman scaling formalism; and the other cross sections are obtained from MARS15 [99] simulations.

The Sanford-Wang parameterization [100] of the inclusive production cross section is given by

$$
\frac{d^{2} \sigma}{d p d \Omega}=c_{1}\left(1-\frac{p}{p_{B}-c_{9}}\right) \exp \left[-c_{3} \frac{p^{c_{4}}}{p_{B}^{c_{5}}}-c_{6} \theta\left(p-c_{7} p_{B} \cos ^{c_{8}} \theta\right)\right]
$$

where $p$ and $\theta$ are the meson momentum and angle, $p_{B}$ is the beam momentum, and the constants $c_{1}, \ldots, c_{9}$ are empirical parameters determined from fits to meson production data. The parameters used in MiniBooNE come from fits to BNL E910 [101] $\left(p_{B}=6.4\right.$, 12.3, and $17.6 \mathrm{GeV} / \mathrm{c} ; 0.4<p<5.6 \mathrm{GeV} / \mathrm{c} ; 18<\theta<400 \mathrm{mrad})$ and CERN HARP [102] $\left(p_{B}=8.9 \mathrm{GeV} / \mathrm{c} ; 0.75<p<6.5 \mathrm{GeV} / \mathrm{c} ; 30<\theta<210 \mathrm{mrad}\right)$. These experiments have good coverage of the entire MiniBooNE phase space. 


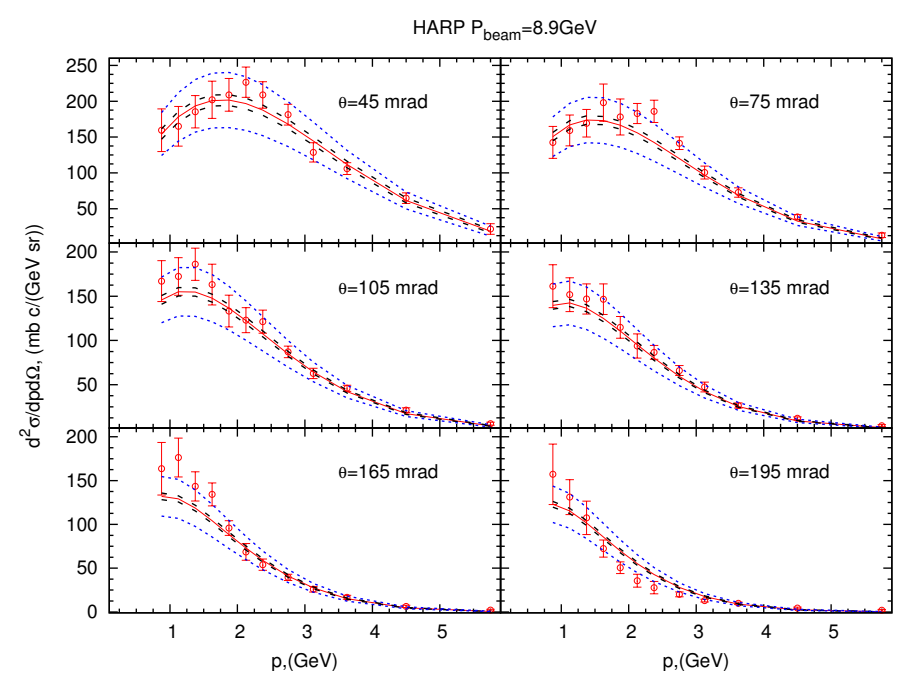

Figure 4.1: HARP $\pi^{+}$data (points with errors) shown with Sanford-Wang fits (central solid line in each panel) at various pion angles. The outer dashed lines represent uncertainties assigned to these cross sections. Plots come from [103.

Figure 4.1 shows the HARP $\pi^{+}$data with Sanford-Wang fits. These HARP data are particularly relevant since they occur at the same $p_{B}$ and with the same beryllium target as MiniBooNE. Reference [104] provides a detailed discussion of the HARP experiment and its relevance to MiniBooNE.

The Feynman scaling parameterization of the $K^{+}$cross section [105] depends only on the transverse momentum $p_{t}$ of the outgoing $K^{+}$and the Feynman scaling parameter $x_{F}=$ $p_{\|}^{C M} / p_{\|}^{C M, \max }$ (where $p_{\|}$is longitudinal momentum):

$$
\frac{d^{2} \sigma}{d p d \Omega}=\frac{p_{K}^{2}}{E_{K}} c_{1}\left(1-\left|x_{F}\right|\right)^{c_{3}} \exp \left[-c_{3}\left|x_{F}\right|^{c_{4}}-c_{7}\left|p_{t} * x_{F}\right|^{c_{6}}-c_{2} p_{t}-c_{5} p_{t}^{2}\right]
$$

where the constants $c_{1}, \ldots, c_{7}$ are fit to external [106-114] data that cover the majority of MiniBooNE's parameter space (figure 4.2.

These $K^{+}$as well as the other mesons produced via the primary proton interactions are then tracked until they decay. Those that decay to neutrinos are routed to the second 


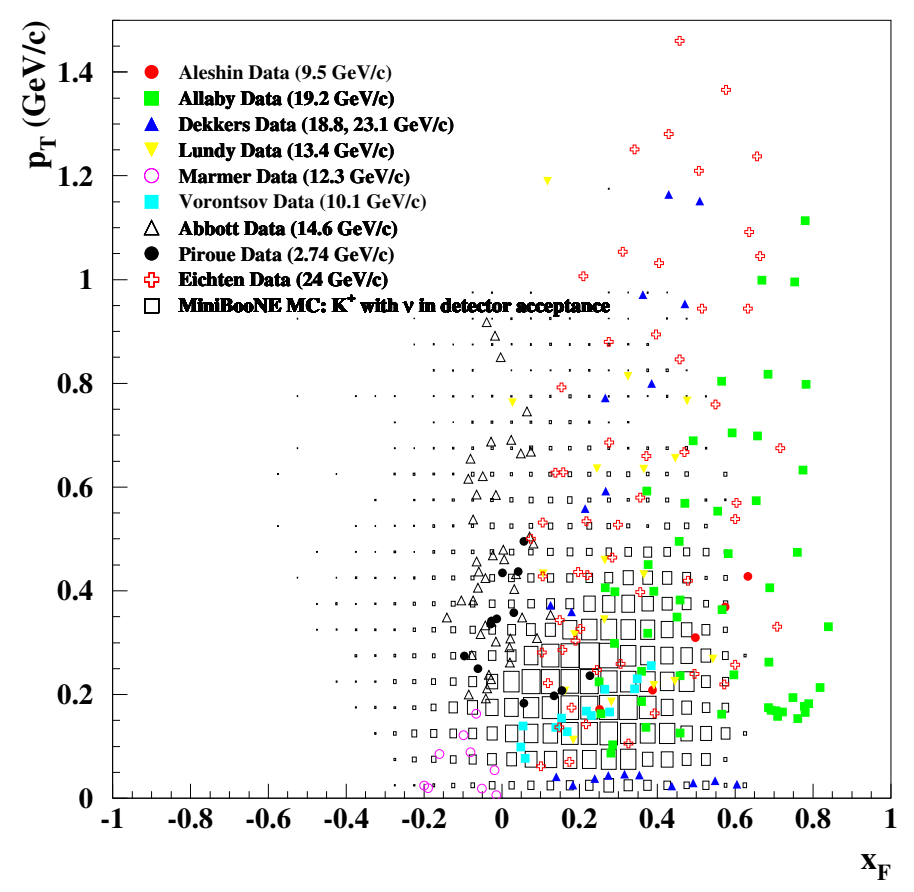

Figure 4.2: Transverse momentum $p_{t}$ vs $x_{F}$ for MiniBooNE $K^{+}$production phase space. The open squares show the MiniBooNE beam Monte Carlo prediction. The other markers indicate the external data.

program that re-decays them 1000 times. This process maximizes the number of final neutrinos simulated for the available computing power, and even allows neutrinos from rare interactions to be produced at a statistically significant level.

At this point in the simulations, the neutrino flavor composition is $93.6 \% \nu_{\mu}, \sim 5.9 \% 1$ $\bar{\nu}_{\mu}, \sim 0.5 \% \nu_{e}$, and less than $0.1 \% \bar{\nu}_{e}$. The majority $(\sim 97 \%)$ of $\nu_{\mu}$ events come from decays of $\pi^{+}$with $K^{+}$contributing most of remaining $3 \%$. The final program constructs the fluxes for each of these neutrino species (as in figure 4.3) and inputs them into the neutrino cross sections simulation code.

\footnotetext{
${ }^{1}$ Cross sections further reduce this contribution to $\sim 2 \%$
} 


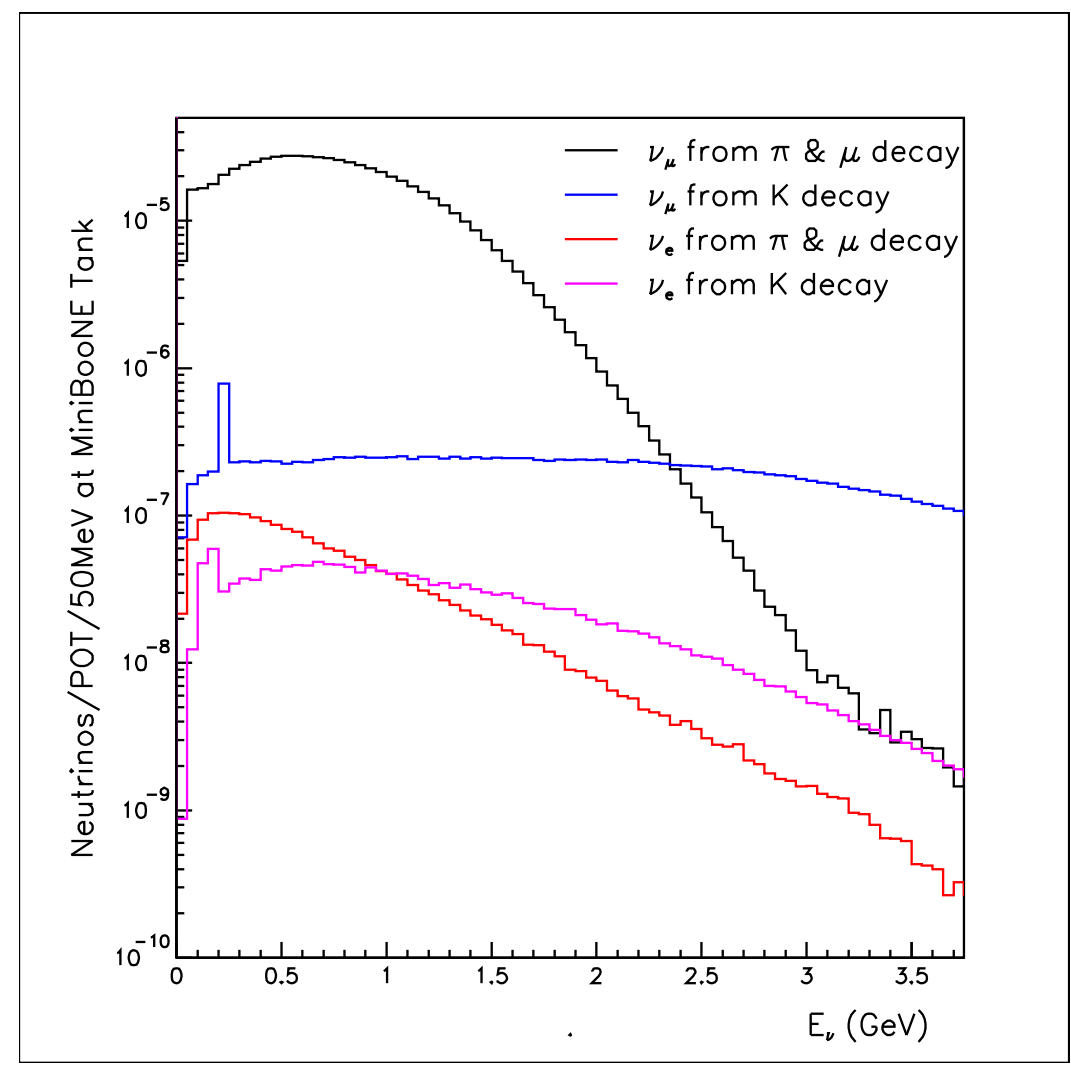

Figure 4.3: Predicted $\nu_{\mu}$ and intrinsic $\nu_{e}$ fluxes as a function of neutrino energy. 


\subsection{Neutrino Interactions Simulation}

Neutrino interaction simulations in MiniBooNE are executed by the FORTRAN-based Nuance cross section Monte Carlo and event generator [115]. The code was originally developed to simulate atmospheric neutrino interactions in the IMB detector and was later modified for use by Super-K and K2K. Several other existing and proposed experiments have further adapted Nuance for their own uses including SNO, KamLAND, MINOS, T2K, MINERvA, NoVA, FINeSSE, SciBooNE, and of course MiniBooNE.

In MiniBooNE, Nuance receives as input the predicted neutrino fluxes and the detector configuration. It then outputs a neutrino interaction rates file and generates events for each input neutrino flavor and 99 separate interactions in an energy range from $10^{-1}$ to $10^{3} \mathrm{GeV}$. The Nuance interaction code is based on the following models:

- the Llewellyn-Smith expression for the quasi-elastic cross section on free nucleons [60],

- the Smith-Moniz relativistic fermi gas (RFG) model for scattering from nucleons bound in a nucleus [116],

- the Rein and Sehgal calculations of resonance cross sections [117], and

- the Bodek-Yang deep inelastic scattering (DIS) cross section at large invariant mass $W$ and momentum transfer $Q^{2}$.

A complete description of the Nuance implementation in MiniBooNE exists in reference [118. The discussion presented here focuses only on the interactions relevant to this analysis.

\subsubsection{Quasi-Elastic Scattering}

Both charged current and neutral current two-body neutrino scattering from nucleons constitute quasi-elastic scattering in Nuance. 


\section{Charged Current Scattering}

Charged current quasi-elastic scattering (CCQE) $\nu_{\mu} n \rightarrow \mu^{-} p$ dominates neutrino interactions at MiniBooNE energies and composes $40 \%$ of observed events in the detector, more than any other channel. The only parameters necessary to describe CCQE scattering off a free nucleon are the form factors described in Chapter 2. Except for the axial mass $M_{A}$ (and the strange quark contributions), these are all well known. $M_{A}$, which is measured from neutrino experiments, is the largest source of theoretical error in calculating the free nucleon cross section.

Calculating the CCQE cross section from nucleons bound in a nucleus is more fraught with error since there is not much data to constrain the many theoretical predictions. MiniBooNE models nuclear effects with a custom-modified [119, 120] Smith-Moniz RFG which includes several effects that alter the interaction:

- Fermi motion of the nucleons in the nucleus does not significantly alter the total cross section, but instead changes the kinematics of the interaction.

- Pauli blocking prevents nucleons from transitioning to energy states in the nucleus that are filled by other nucleons. The reduction in phase space available to final state protons supresses the cross section.

- Nuclear binding between nucleons in the nucleus also supresses the cross section.

Thus the overall result of nuclear effects is to suppress the cross section.

In this neutral current elastic analysis, CCQE events enter only as a $\sim 1 \%$ background. However, the formalism for charged current quasi-elastic and neutral current elastic scattering is essentially the same. 


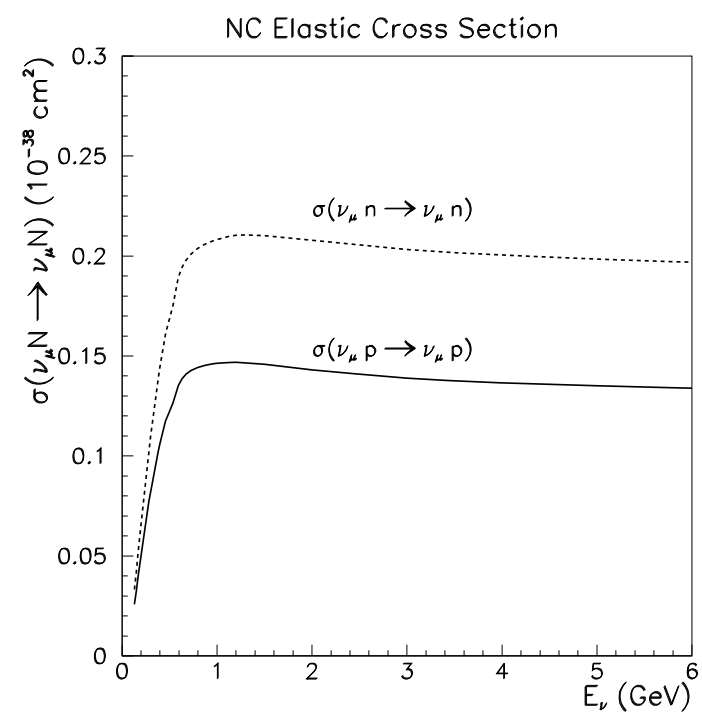

Figure 4.4: Neutral current elastic proton and neutron Nuance cross sections as a function of energy (from [118]).

\section{Neutral Current Scattering}

Though neutral current scattering from a free nucleon $\nu_{\mu} N \rightarrow \nu_{\mu} N$ is an elastic process, scattering from nucleons bound in a nucleus is quasi-elastic, and the interactions are sometimes called "neutral current quasi-elastic". These are the signal interactions for this analysis. Neutral current elastic (NCE) scattering accounts for $\sim 15 \%$ of observed events in MiniBooNE. The formalism differs from CCQE only in that the approximation that the out-going lepton mass is zero is an identity for NCE scattering (Chapter 2).

Scattering from protons and neutrons contribute approximately equal fractions to this interaction. Although the cross section is $\sim 40 \%$ larger for neutrons than protons (figure 4.4), there are $\sim 33 \%$ more protons in the $\mathrm{CH}_{2}$ target molocules.

Naively, only scattering from protons is visible in MiniBooNE via scintillation or Cherenkov radiation. However, neutrons produce light indirectly either by re-scattering on 
protons within the nucleus or by scattering on other nuclei once they escape. Thus both neutral current proton and neutral current neutron events are predicted to contribute to the observed event sample. Moreover, the ways neutron events produce light are so similar to those of proton events that there is currently no method to distinguish between them. Chapter 6 includes a discussion of the efficiencies of detecting neutral current proton and neutral current neutron events (figure 6.14).

\subsubsection{Single pion production}

In the current version of Nuance, single pion production accounts for the only significant predicted neutrino background to the NCE analysis other than CCQE. Contributions to single pion reactions come from both resonant and coherent production.

There are three charged current resonant $1 \pi$ channels $\left(\nu_{\mu} p \rightarrow \mu^{-} p \pi^{+}, \nu_{\mu} n \rightarrow \mu^{-} p \pi^{0}\right.$, $\left.\nu_{\mu} n \rightarrow \mu^{-} n \pi^{+}\right)$and four neutral current resonant $1 \pi$ channels $\left(\nu_{\mu} p \rightarrow \nu_{\mu} p \pi^{0}, \nu_{\mu} p \rightarrow \nu_{\mu} n \pi^{+}\right.$, $\left.\nu_{\mu} n \rightarrow \nu_{\mu} n \pi^{0}, \nu_{\mu} n \rightarrow \nu_{\mu} p \pi^{-}\right)$. These reactions occur via production of a nucleon resonance $\left(\Delta\right.$ or $\left.N^{*}\right)$. The dominant contribution to single pion production at these energies is from the $\Delta(1232)$, however all known resonances with invariant mass $<2 \mathrm{GeV}$ are included in Nuance.

The other contribution to single pion production comes from coherent production. This occurs when the neutrino scatters from an entire nucleus $A\left(\nu_{\mu} A \rightarrow \mu^{-} \pi^{+} A, \nu_{\mu} A \rightarrow \nu_{\mu} \pi^{0} A\right)$. These interactions do not break up the nucleus. In fact, the nucleus as a whole remains in its ground state without changing its charge or isospin. Coherent production accounts for only a small fraction $(\sim 5 \%)$ of the total neutrino cross section at neutrino energies aroung $1 \mathrm{GeV}$.

Of single pion production, neutral current $1 \pi$ is the largest background to the NCE analysis at $\sim 15 \%$. This is mainly due to certain final state interactions discussed next. Charged current $1 \pi$ and coherent pion production are much smaller backgrounds together 
with multi-pion channels (not discussed here) together making up only $\sim 1 \%$ of neutrino interactions in this analysis.

\subsubsection{Final State Interactions}

"Final state interactions" (FSI) is a general term used here to describe any re-interaction of the original particles produced by neutrino interactions in carbon nuclei before they leave the nucleus. Such processes can completely transform the the observed final state of an event.

Nuance simulates FSI by tracking hadrons through the nucleus in steps of $0.2 \mathrm{fm}$. At each step it calculates an interaction probability based on measured $\pi-N$ and $N-N$ cross sections and angular distributions. This method allows particles to interact several times before leaving the nucleus. Nuance also simulates nuclear de-excitation by allowing the nucleus to transition to a higher energy level and promptly decay to its ground state via emission of several-MeV photons.

Final state interactions for pions include absorption, charge exchange (e.g. $\pi^{+} n \rightarrow \pi^{0} p$ and $\pi^{-} p \rightarrow \pi^{0} n$ ), and elastic and inelastic scattering and re-scattering. Pion absorption is expected approximately $20 \%$ of the time in Nuance, and charge exchange $\sim 10 \%$. These processes can also occur at the detector modeling stage in the simulation chain with final state particles in the volume of the detector. However, the affects of absorption and charge exchange at that stage of simulation have not been investigated here since the Nuance processes are dominant in this analysis. Errors on these processes from both simulations are included in the final results.

Scattered nucleons also incur FSI. While roughly $1-2 \%$ of nucleon re-interactions produce a pion, nucleons are much more likely to re-scatter and produce additional nucleons. In this way, neutrons scattering off protons are a mechanism by which neutral current neutron events generate light in the detector. 
Another component of FSI is the production of several-MeV photons from nuclear deexcitation. This occurs most often in resonant pion production channels.

Because final state interactions can completely obscure the original interaction type, MiniBooNE typically classifies events according to the final state: what is actually observed in the detector. Therefore, an event with any number of neutrons, protons, and soft photons, but no leptons or pions would be "NCE-like". Since Nuance simulated FSI tend to suppress pion production, it is more common for a true single pion event to be classified "NCE-like" than for a true NCE event to be classified as " $1 \pi$-like". This accounts for the majority of the single pion background entering the NCE sample.

Once particles from neutrino interactions have escaped the nucleus, relevant information about them (such as their energies and momenta) is directed to the detector response simulation.

\subsection{Detector Simulation}

The detector Monte Carlo is a GEANT3-based program that simulates the MiniBooNE detector response to particles as they travel through the materials in and around it. A general overview of this process is presented here; further details may be found in reference 121.

The Monte Carlo code models the detector geometry as it is described in Chapter 3 with the addition of a large (14 m radius) cylinder of surrounding material (dirt) as in figure 4.5 . This additional volume is included because it is possible for neutrinos interacting in the surrounding dirt to produce particles that enter the detector. While many of these particles should produce light in the veto region, some can fake the signature of an event created within the detector. These events are a large background for the NCE analysis.

The detector Monte Carlo distributes the Nuance input events throughout the simulation 


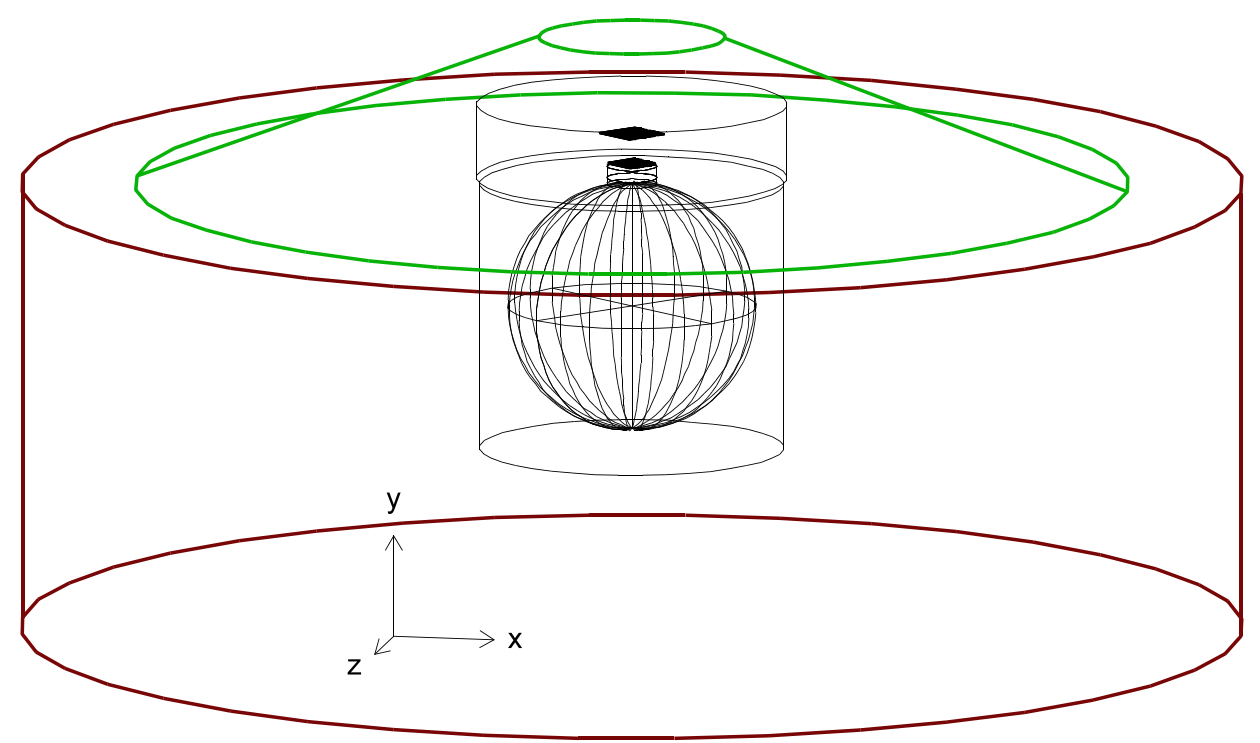

Figure 4.5: GEANT3 geometry for the detector, vault, electronics area, overburden, and surrounding dirt.

volume weighted by the local density. Thus the surrounding $\operatorname{dirt}\left(\rho=2.15 \mathrm{~g} / \mathrm{cm}^{3}\right)$ receives more interactions per unit volume than the oil $\left.\left(\rho=0.855 \mathrm{~g} / \mathrm{cm}^{3}\right)\right|^{2}$

Once the event vertices are established, the Monte Carlo propagates the final state particles through the simulation volume accounting for relevant physical and optical phenomena (e.g. particle decay or capture, scattering, hadronic phenomena including pion absorption and charge exchange (modeled here by GCALOR [122]), dE/dx, Cherenkov and scintillation emission). All photons emitted by primary final state particles, their decay products, or scattered particles are tracked from the point of origin until they are absorbed via any of the phenomena discussed in Chapter 3. Of course, absorption also occurs at PMT photocathodes but with a probability to produce a photoelectron.

For each particle produced in the detector Monte Carlo, the program records relevant

\footnotetext{
${ }^{2}$ It is interesting to note that although the density of the dirt is handled correctly, the dirt itself is modeled as carbon nuclei.
} 
"truth" information such as the particle vertex and initial momentum. The simulation also stores the number of scintillation and Cherenkov photons that were created and their arrival times at the PMT photocathodes.

Once photoelectrons are generated at the PMT cathodes, a separate program that models the data acquisition (DAQ) system takes over simulation of these events. The purpose of the DAQ simulator is to make events from the Monte Carlo look just like the data so that both can be treated equally by further processing. To that end, the DAQ simulator smears photoelectron charge and time at the photocathode to determine these quantities at the anode. Then for each channel (just like the real data acquisition described in Chapter 3), it fires a discriminator when the charge threshold is crossed, loads data into time and charge "quads", and simulates how this data is recorded to tape. The resultant information is then calibrated according to the same procedure as real events. Processed in this way, Monte Carlo events exactly mimic the uncalibrated data with the addition that the particle truth information is still intact.

The final step for simulated events is the introduction of neutrino unrelated backgrounds (e.g. cosmic ray events and PMT dark noise). Instead of simulating such backgrounds, each Monte Carlo event is merged with a data event from the $2.01 \mathrm{~Hz}$ random (strobe) trigger. However, these merged events are processed only if the neutrino interaction produced light in a PMT, otherwise they are discarded. Since there are many more beam triggers without a neutrino event than with (let alone a neutrino event that produces light), this procedure necessarily underestimates the amount of beam unrelated background that ought to be present in a given sample of Monte Carlo. However this issue is addressed for the neutral current elastic analysis using particle ID (Chapter 5). 


\subsection{Simulation Uncertainties}

All of the simulations use models with inherent uncertainties that must be accounted for. These uncertanties are described separately here for each step of the total simulation: beam and flux, neutrino interactions, and detector.

\subsubsection{Beam and Flux Uncertainties}

This account of beam and flux uncertainties draws from reference [123]:

- The primary source of beam/flux related errors in the NCE analysis comes from correlated uncertainties of the $\pi^{+}$production Sanford-Wang parameters $\left(c_{1}, \ldots, c_{9}\right)$ determined in the fits discussed in section 4.1 .

- Uncertainties on the Sanford-Wang parameters for $\pi^{-}$and $K_{0}$ production as well as on the Feynman scaling parameters for $K^{+}$also contribute.

- The secondary source of beam/flux errors comes from uncertainties of the total $N-N$ and $\pi-N$ hadronic cross sections in beryllium as well as the kinematics of inelastic and quasi-elastic scattering processes in these interactions.

- Uncertainties in the horn skin depth and horn current are also considered.

The error on the measurement of protons on target $(\sim 2 \%$ from [124]) is considered separately as a normalization error on the NCE cross section measurement (Chapter 6).

\subsubsection{Neutrino Interaction Uncertainties}

For the neutrino interaction model, MiniBooNE attempts to constrain the uncertainties using external data wherever possible. In situations where little or no external data is 
available, uncertainties are constrained by comparing the output of Nuance predictions with MiniBooNE data [125].

- Uncertainties on the free nucleon cross sections enter via the axial masses $M_{A}$ (in $\mathrm{GeV}$ ) for those cross sections. For quasi-elastic: $M_{A}^{Q E}=1.23 \pm 0.20$, for resonant single pion production: $M_{A}^{1 \pi}=1.1 \pm 0.3$, for resonant multi pion production (not discussed) $M_{A}^{N \pi}=1.3 \pm 0.5$, and for coherent production $M_{A}^{c o h}=1.03 \pm 0.3$. The error on $M_{A}^{Q E}$ comes from MiniBooNE data, the rest from external data.

- Uncertainties in the relativistic fermi gas (RFG) model enter through its parameters. The nucleon binding energy and fermi momentum errors come from external (electron scattering) data. However, a parameter $\kappa$ that modifies the lower integration limit of the Smith-Moniz Pauli blocking model is determined from MiniBooNE data: $\kappa=$ $1.019 \pm 0.011$.

- Final state interaction uncertainties are handled with the pion absorption and charge exchange cross sections and $\Delta N \rightarrow N N$ interaction probability in Nuance. These have been determined from external data.

- Further errors determined from MiniBooNE data include the $\mathrm{NC} \pi^{0}$ rate as a function of momentum, the $\mathrm{NC} \pi^{0}$ coherent to resonant ratio, and the rate and branching fraction of radiative $\Delta$ decay.

- One important uncertainty for the $\mathrm{NCE}$ analysis is the uncertainty on $\Delta s$. Variations in this quantity change the ratio of neutral current proton to neutral current neutron interactions in the model. The uncertainty on $\Delta s$ comes from external data: $0.0 \pm 0.1$.

\subsubsection{Detector Simulation Uncertainties}

Like Nuance, the detector simulation and its uncertainties are based on external data as well as comparisons to MiniBooNE data. Most detector Monte Carlo parameters were tuned 
within their uncertainties to try to match simulated and real events in observables drawn from non-oscillation samples of MiniBooNE data.

A few parameters were tuned individually:

- Uncertainties on the hadronic model used in MiniBooNE (GCALOR) were assessed by comparing the results to the GFLUKA model.

- The PMT discriminator threshold is nominally $0.1 \mathrm{PE}$, and was varied by $\pm 0.1 \mathrm{PE}$.

- Charge-time correlations were also tuned separately.

Other parameters were not tuned at all:

- The uncertainty on the PMT prompt timing response was determined from subnanosecond discrepancies between the time distributions of Michel electrons and muons that stopped in the cubes.

- Certain particle propagation uncertainties are set at fixed values: e.g. bremsstralung and Compton scattering are each assigned a $5 \%$ uncertainty.

However the majority of parameters with tunable errors in the detector Monte Carlo come from the photon propagation model. Many of these parameters affected several observable variables at once and were also correlated with each other. MiniBoonE developed a system that allowed tuning the parameter space of all (35) relevant variables simultaneously using observables from Michel electron data to constrain uncertainties. This procedure is described in reference [121]:

- The most important sources of error in the light propagation model are the scintillation and fluorescence yields and their time constants. 
- Other important parameters were the Cherenkov scale factor, the index of refraction, the extinction length, and the parameters of Birk's law.

- Rayleigh and Raman scattering, reflection probabilities, and the PMT angular efficiencies were also considered.

- Also an uncertainty was determined for the efficiency of old to new PMTs.

Data from the neutral current analysis played a role in constraining some of these variables (notably scintillation and fluorescence yields).

\subsubsection{Dirt Neutrino Model Uncertainties}

The model for neutrino interactions in the dirt and the propagation of final state particles in the detector is necessarily more simple than the reality of the physics that takes place. An example already mentioned is that the simulation treats the dirt as carbon nuclei but with the correct density for dirt. Such approximations are practical. The volume through which dirt events must be generated and tracked is huge, and even these simplified models significantly increase computation time for the simulations.

Furthermore, dirt events are not a significant background for most samples because the particles that enter the tank tend to have low visible energy. Unfortunately for that very reason, it is the largest background for the NCE analysis. Therefore, uncertainties on the dirt model are determined specifically for this analysis. They include an uncertainty on the normalization and on the reconstructed energy shape of the dirt background. Both of these will be discussed further in Chapter 5 . 


\subsection{Event Reconstruction: Subevents}

After Monte Carlo events have passed through the simulation chain, they have the same properties as data events (with the addition of "truth" information). Further processing is now identical for both simulated and real events.

The first step in continued processing is the SplitEvent algorithm. This program locates and separates interactions within a trigger window by identifying time-separated clusters of PMT hits [126]. Each cluster is made up of a group of at least 10 PMT hits from the main detector, veto, or both that are separated by at least 10 ns. These separated hits clusters are called "subevents" in this analysis.

One of the main purposes of this algorithm is to tag events with particles that decay. For example in many CCQE events $\left(\nu_{\mu} n \rightarrow \mu^{-} p\right)$, the muon stops in the detector and decays into a Michel electron. Such an event should have two distinct subevents: the first from the muon and the second from the Michel. However a neutral current elastic event $\left(\nu_{\mu} N \rightarrow \nu_{\mu} N\right)$ should have only a single cluster of hits from the outgoing nucleon. This makes the subevents a powerful tool for identifying interaction types.

Subevents are processed by this and other algorithms as if they were miniature events. For example, SplitEvent assigns each subevent the average PMT hit time (AvgTTim), number of veto PMT hits (VHits), number of main detector PMT hits (THits) ${ }^{3}$, and other quantities calculated specifically for that cluster of hits. Event information organized into collections of subevents is then passed as input to the reconstruction algorithms.

\footnotetext{
${ }^{3}$ The "T" stands for "Tank" which is the common abbreviation for the main volume of the detector.
} 


\subsection{Event Reconstruction: Fitting Events}

The event reconstrution algorithms used for this analysis come from the StancuFitters suite developed by Ion Stancu for MiniBooNE. Events are processed by three separate algorithms: the Stancu Fast Fitter, the Stancu Full Fitter, and the Stancu Flux Fitter. The account described here draws heavily from [127] and also from [128], however further details may be found in reference [129]. In all of these routines, minimization/maximization is performed by MINUIT [130].

\subsubsection{Stancu Fast Fitter}

The Fast Fitter is the initial stage of event reconstruction. This algorithm determines the initial event 4 -vertex $T_{0}$ and $\vec{R}_{0}$ from the charge weighted position of the PMTs. It then calculates the "corrected" 4 time for each hit PMT $i$ :

$$
\Delta t_{i}=t_{i}-T_{0}-\frac{1}{c}\left|\vec{r}_{i}-\vec{R}_{0}\right|
$$

where $t_{i}$ and $\overrightarrow{r_{i}}$ are the time and position of PMT $i$. The code uses these times to construct a corrected time distribution that is the convolution of a Gaussian plus a $\delta$-function and an exponentially delayed component. This time likelihood function is maximized with respect to $T_{0}$ and $\vec{R}_{0}$.

The Fast Fitter also determines an initial event direction from the charge weighted direction cosines of prompt-hit PMTs with respect to the event vertex. Prompt hits typically come from Cherenkov radiation which is directional, unlike delayed scintillation light which is isotropic. The initial energy of the event is calculated from the total collected charge corrected for the shortest distance of the event to the edge of the main detector volume.

\footnotetext{
${ }^{4}$ The time is corrected since it accounts for the distance from the event vertex to the phototube and the flight time of the light.
} 
Finally, this algorithm determines initial scintillation and Cherenkov fluxes from the calculated energy using proportionality constants extracted from Michel electron data.

\subsubsection{Stancu Full Fitter}

In the second stage of reconstruction, the Cherenkov and scintillation fluxes are fixed to their values from the Fast Fitter. The angular distribution of Cherenkov light $F(\cos \theta)$ is determined subject to the normalization

$$
\int_{-1}^{1} F(\cos \theta) d \cos \theta=1
$$

Then the event 4-vertex and direction are extracted from a more sophisticated time and charge likelihood. The charge portion of the likelihood assumes the event is an electron and then uses the current fit position and direction to calculate the expected charge on each tube. A new energy is calculated as in the Fast Fitter but for the new shortest distance to the edge of the detector volume.

\subsubsection{Stancu Flux Fitter}

The Flux Fitter re-performs the Full Fit, however now the 4-vertex and directions are fixed (to their Full Fit values) and the relative strengths of the scintillation and Cherenkov fluxes are allowed to float.

Each flux is proportional to the total number of generated photons for that type of light. Using scintillation light as an example, the number of generated scintillation photons is given by

$$
N_{\gamma}=\frac{4 \Phi}{\epsilon R^{2}}
$$

where $\Phi$ is the reconstructed scintillation flux, $\epsilon$ is the absolute quantum efficiency, and $R$ is the area of the PMT normal to the light direction. For reasonable values of $\epsilon=0.25$ and $R=0.1016$, the scintillation flux is $N_{\gamma}=1550.0 \Phi$. 
To compare the reconstructed scintillation flux with the reconstructed Cherenkov flux, the Cherenkov flux must be scaled down by a factor of two. The normalization of $F(\cos \theta)$ from equation 4.4 (which is used for historic reasons) should really be

$$
\frac{1}{2} \int_{-1}^{1} F(\cos \theta) d \cos \theta=1
$$

for direct comparisons to the scintillation flux.

For example, to determine the fraction of scintillation flux in an event $f_{s}$, the correct equation is

$$
f_{s}=\frac{\Phi}{\Phi+\rho / 2}
$$

where $\rho$ is the Cherenkov flux.

It is important to note that the algorithms described here were designed for an electron hypothesis. That they work reasonably well for nucleons is a testament to their overall robustness. However a new suite of fitters called the NCFitters has been designed in the same vein as the StancuFitters but implementing a proton hypothesis. These new algorithms are being tested on neutral current events and will be the subject of future analyses. 


\section{Chapter 5}

\section{Data Selection}

Neutral current elastic events comprise $\sim 15 \%$ of the MiniBooNE data set. The signature for these events is fundamentally different from other interactions because they generate nearly pure scintillation light at low energies. However, these differences allow a simple event selection to produce a high quality set of NCE events.

This chapter describes the selection processes that yield the event sample used to calculate a neutral current elastic cross section in MiniBooNE.

\subsection{Data Quality}

The first stage of selection guarantees basic data quality at all for the entire chain of data production beginning with the beamline hardware and ending with events being written to disk. The first actual selection ensures that the event was not "latent." It removes events where data in the DAQ circular buffers were overwritten because the trigger failed to decide whether to keep the event (Chapter 3). Only $0.1 \%$ of the events in the NCE data set were latent. 
Other selections guarantee that the beam hardware was operating within normal parameter limits. These include ensuring that a minimum number of protons were in the beam line, that the beam was on target, that the horn current was nominal, and others. Approximately $63 \%$ of non-latent beam triggers entering the neutral current sample passed beam data quality selections.

Data quality checks are also performed for detector hardware. Problematic PMT channels (PMTs with bad charge gain, timing slope, delay, noise spikes, or that no longer output a signal) are removed from both data and Monte Carlo samples for all events. There are 34 of these channels. Additionally, data runs are removed if run logs indicate that the experiment was not operating under optimal conditions. However, all runs in the range used for this analysis (runs 3539-5299) were of good quality.

After data quality selections, the total number of protons on target is $(6.57 \pm 0.05) \times 10^{19}$ producing $17.87 \times 10^{6}$ beam triggers sampled in the neutral current elastic analysis. This is $\sim 10 \%$ of the currently published data.

\subsection{Neutral Current Pre-Selection}

Assured of high quality data, further selection focuses on isolating neutral current elastic events. At the most basic level, the signature for NCE events is small amounts of light produced by low kinetic energy protons in time with the beam. These protons can come both from neutrino interactions on the hydrogen, or more often from interactions on either protons or neutrons in the carbon nuclei. Variables from the SplitEvent algorithm (Chapter 4) can be used to pre-select NCE events at this general level:

- To help ensure that events are neutrino induced and contained within the signal region, the number of hit PMTs in the veto region must be consistent with noise: VHits $<6$. 


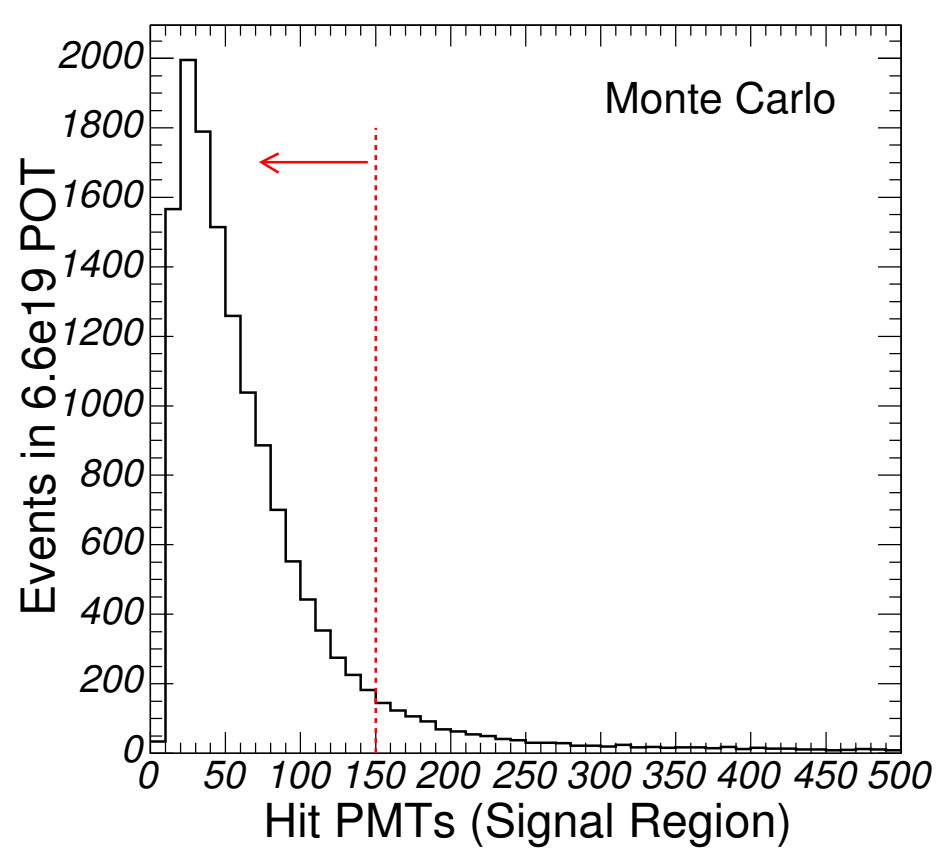

Figure 5.1: Monte Carlo THits distribution for NCE events. The other SplitEvent cuts have been applied. The dashed vertical line indicates the cut at THits $=150$ with $\sim 90 \%$ of NCE events predicted to be in the peak below the cut.

- NCE events should occur as a single subevent. This helps eliminate charged-current events and other events that produce more than one subevent.

- To guarantee that events are in time with the beam, the average hit time of detector PMTs must fall between $4400<$ AvgTTim $<6500$ ns.

- Since these events produce only low levels of light in the detector, the number of hit PMTs in the signal region must be low: THits $<150$.

The value of 150 for the final selection was chosen to preserve blindness for the oscillation sample. Simulations suggested that setting the THits selection higher than 150 would allow too many oscillation events into the sample. However figure 5.1 shows that the choice of 150 
was not restrictive for this analysis, keeping 90\% of events passing the other SplitEvent selections.

After passing this stage of selections, Monte Carlo events generated within the detector are already $\sim 80 \%$ pure neutral current elastic events. Only $\sim 3 \%$ of the backgrounds come from CCQE events, and most of the rest are single pion events.

\subsection{Proton PID \& Beam Unrelated Backgrounds}

The NCE sample after pre-selection is ideal for developing particle identification (PID) for protons. This stage of selection exploits the timing differences between light emitted by protons and other particles. Interpreting the nature of these other particles requires the use of beam-unrelated data from the random (strobe) trigger (Chapter 3) as well as a low energy sample of CCQE events.

\subsubsection{Proton Particle Identification}

\begin{tabular}{l|c|r}
\hline Particle & Mass $[\mathrm{MeV}]$ & Threshold $[\mathrm{MeV}]$ \\
\hline electron & 0.511 & 0.2 \\
muon & 106.0 & 39 \\
pion & 140.0 & 51 \\
proton & 938.0 & 343 \\
\hline
\end{tabular}

Table 5.1: Cherenkov threshold for representative particles in MiniBooNE oil.

Protons have a high Cherenkov threshold compared to other particles commonly produced in MiniBooNE due to their large mass (table 5.1). Therefore visible protons with only a few hundred $\mathrm{MeV}$ kinetic energy emit larger fractions of scintillation light than other particles. Since emission of scintillation light is delayed with respect to particle interaction 
whereas Cherenkov light is prompt, the fraction of prompt light in an event can be used to identify protons. For this analysis, a photon is prompt if its reconstructed PMT hit time is between $-3<t_{\text {hit }}<7$ ns (reconstruction effects allow hit times to be negative).

Testing the effectiveness of this PID is trivial for the NCE data sample at this stage because it still contains beam unrelated background at the $\sim 30 \%$ level. The majority of this background occurs when cosmic ray muons enter the detector before the beam trigger window starts but then decay into a Michel electron during the nominal beam window. Since these electrons have not produced light in the veto, do not decay, and generally produce no more than $\sim 160$ hit PMTs in the signal region, they easily survive the preselection. However, light from Michel electrons is produced with a ratio of 7:3 Cherenkov to scintillation making them good candidates against which to compare protons using the PID.

Figure 5.2 shows the prompt hits fraction distributions for NCE data, Monte Carlo simulated events (absolutely normalized to data POT), and a pure sample of beam unrelated background (from the strobe trigger). The dashed vertical line indicates the separation between proton-like events at low prompt hits fraction on the left and electron-like events at high prompt fraction on the right. The value of the vertical line is 0.55 .

The NCE data splits into both proton-like and electron-like peaks. On the proton side, the Monte Carlo matches the data within systematic errors. To the right, the strobe trigger data peaks at the same location as the data confirming that this portion of data is indeed Michel electrons. The bump of Monte Carlo events in that same location comes from strobe trigger data merged with the Monte Carlo sample (Chapter 4).

Applying a cut to keep events below a prompt hits fraction of 0.55 ensures proton-like events and also reduces the amount of beam unrelated background in the data from $31 \%$ to $2 \%$.

The power of this PID can also be verified with beam neutrino data. A sample of low 


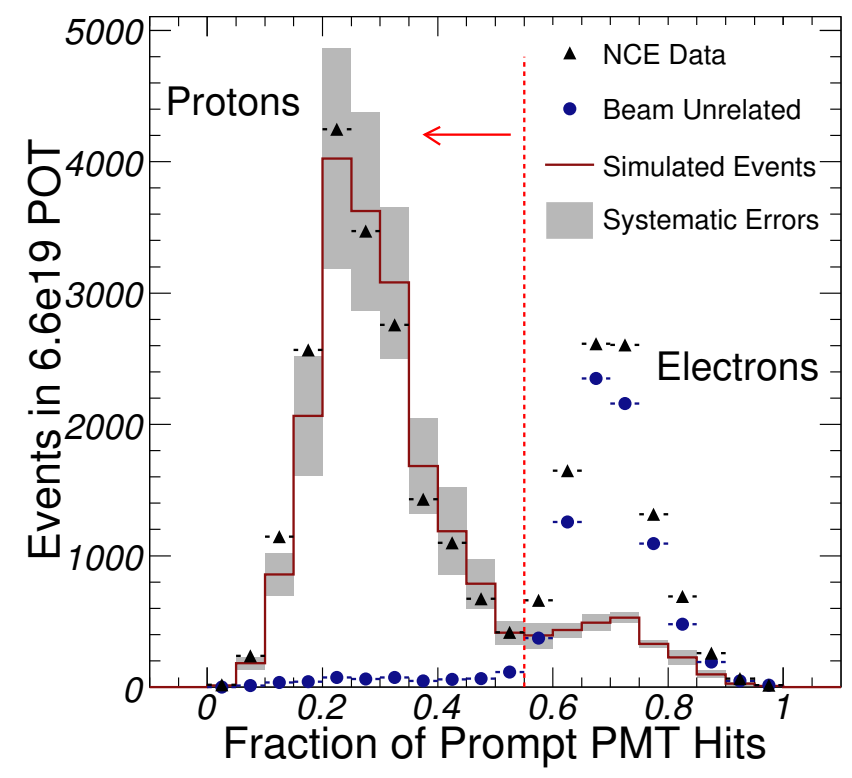

Figure 5.2: Prompt hits fraction as proton particle ID. The dashed line separates protonlike events (left) from electron-like events (right). Monte Carlo is absolutely normalized to the number of protons on target in the data sample.

energy CCQE events $\left(\nu_{\mu} n \rightarrow \mu p\right)$ corresponding to the NCE sample is also open for analysis. These events have the same data quality and pre-cuts as the NCE sample, with the exception that they require exactly 2 subevents (versus 1 for NCE events). The first subevent is the muon, the second is the decay electron.

Figure 5.3 shows the PID variable with the CCQE data overlayed on the NCE and unrelated background data. That the three electron samples all peak in the same place re-confirms the hypothesis that they are in fact electrons. The muon distribution is wide and peaks between the protons and electrons. This is due to how the interaction energy is shared between the final state muon and proton.

When the muon receives the majority of the neutrino energy, it produces mainly prompt 


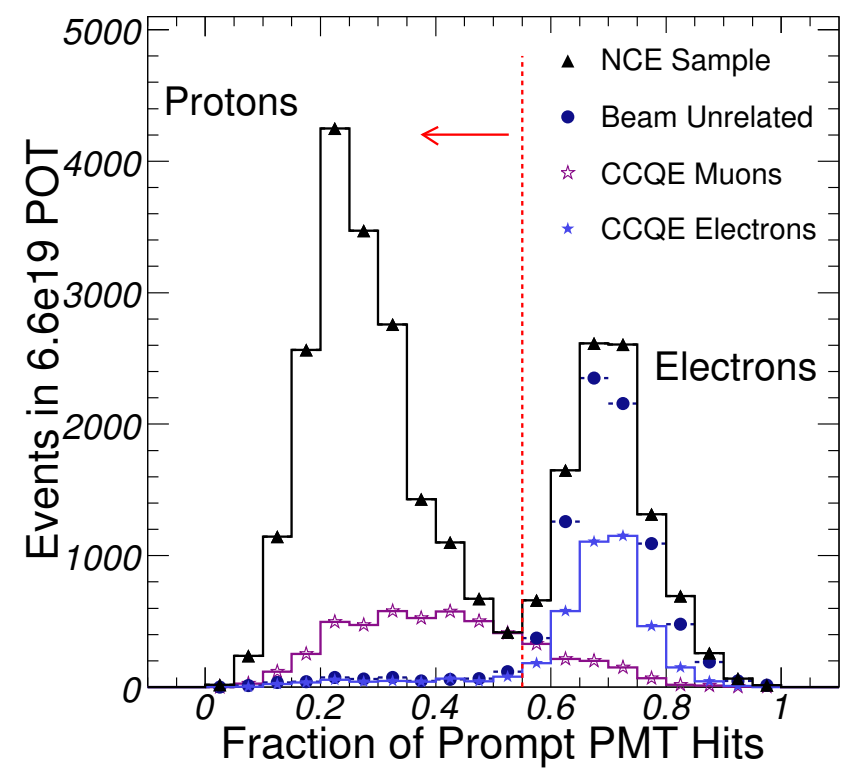

Figure 5.3: Prompt hits fraction with low energy CCQE muon and electron data overlaid on the NCE proton and strobe data.

Cherenkov light and will be reconstructed in the electron peak. As energy is shared with the proton, the fraction of Cherenkov light the muon produces decreases and the fraction of scintillation increases making it reconstruct more like a proton. Regardless, it is clear that protons and muons exhibit different behavior on the whole and therefore provides further evidence that the "proton" peak is indeed hadronic.

\subsubsection{Normalizing The Unrelated Background}

The beam-unrelated activity must be normalized appropriately to be compared to the data, and then subtracted as a background. This activity occurs with equal probability at all times and therefore is equally likely to be sampled by both beam and strobe triggers. Since neutrino interactions are rare and these triggers are of equal length $(19.2 \mu \mathrm{s})$, then the 

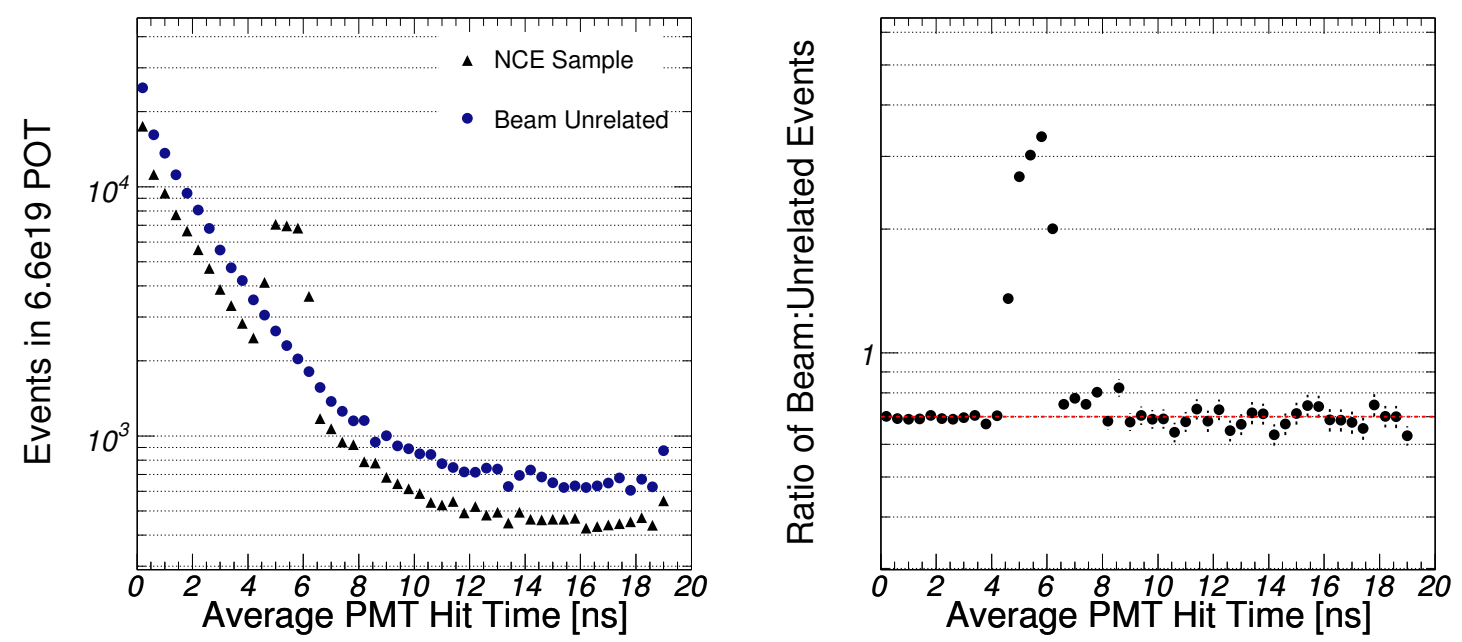

Figure 5.4: Left panel: beam-unrelated and neutrino data samples with pre-selection cuts. There are more strobe than beam triggers that pass cuts. Right panel: ratio of neutrino to beam-unrelated events. Outside the beam window this is fit to $\sim 0.7$ (the line at 0.7 guides the eye).

amount of unrelated activity in $N$ beam triggers is the same as in $N$ strobe triggers. This implies that the unrelated backgounds should be scaled by the ratio of strobe to beam triggers. Before applying pre-selection cuts, there are 17.9 million beam and 25.6 million strobe triggers resulting in a scale factor of 0.7 .

To ensure that this scale factor is valid, the two data samples are compared in time. The left panel of figure 5.4 shows neutrino data along with unrelated activity for the full length of the trigger window. The tower in the NCE data indicates the presense of neutrino data. The ratio of beam to strobe data is shown in the right panel of the figure. Fits to this distribution on either side of the beam window indicate the ratio is flat at a value of 0.7. This agrees with the naive trigger normalization and is applied to the beam unrelated backgrounds in figures 5.2 and 5.3 .

Figure 5.5 shows this same information after the PID selection has been made. Now 

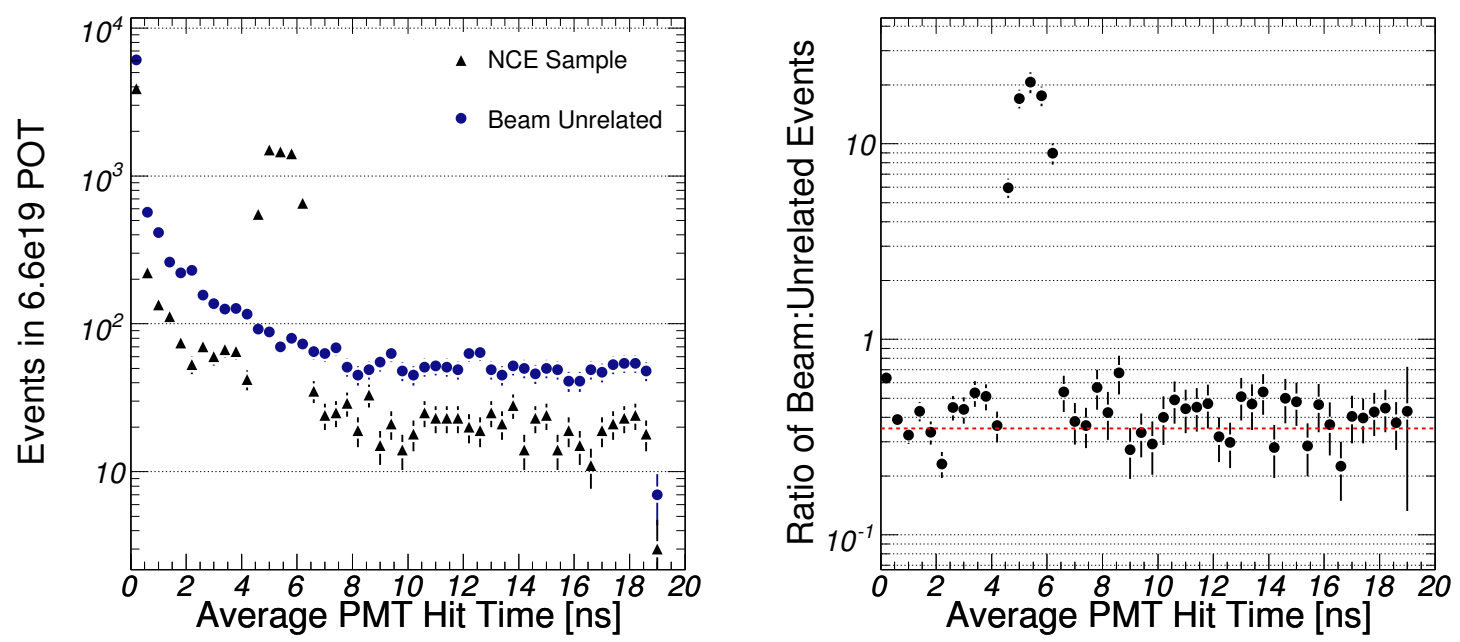

Figure 5.5: Left panel: beam-unrelated and neutrino data samples with all selections (including radius selection defined in cut 5.4. Right panel: the ratio is fit to $\sim 0.35$ outside the beam window (the line at 0.35 is to guide the eye).

fits to the ratio indicate a lower value of 0.35 . This discrepancy has been investigated but is not fully understood. A $100 \%$ error is applied to this scale factor to cover the difference between it and the naive expectation. However, the background is small (a few percent), and so this is not a dominant error. This normalization will be applied to the beam-unrelated background subtraction presented in Chapter 6 .

\section{4 "Dirt" Backgrounds}

Despite pre-selection and particle ID, there is an insidious background that penetrates the entire NCE sample. As mentioned in Chapter 4, it is possible for neutrinos from the beam to interact in the dirt surrounding the detector and eject particles (mainly neutrons in this analysis) that travel into the signal region to produce light. Because this background mimics the signal, it is necessary to rely on the Monte Carlo simulations to predict them. 


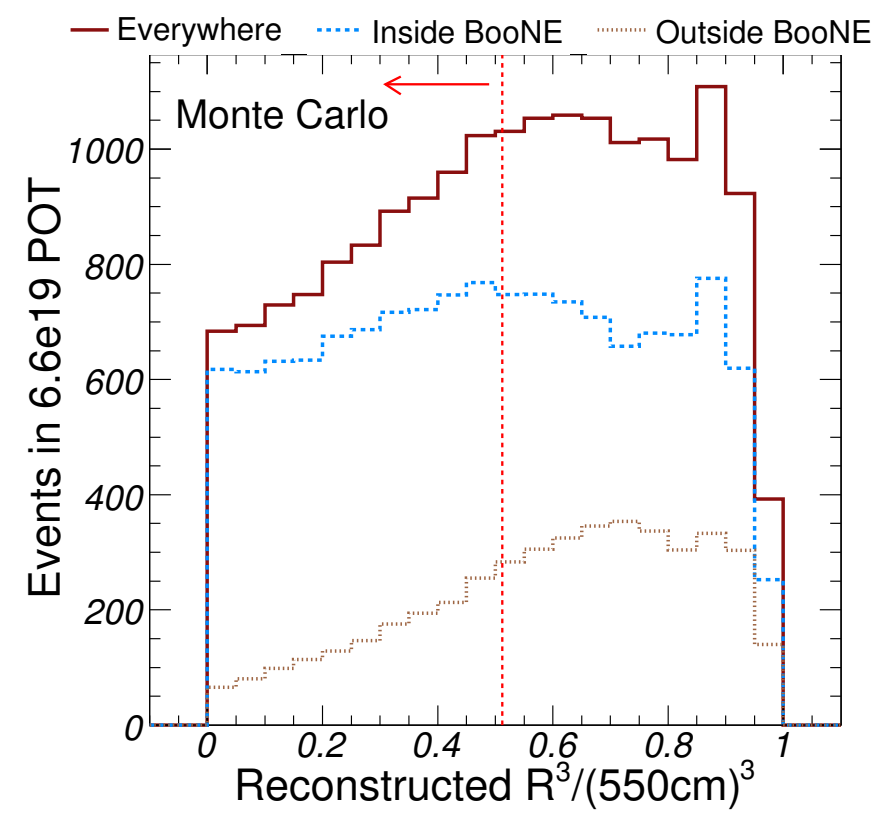

Figure 5.6: Reconstructed $R^{3}$ normalized to $550 \mathrm{~cm}^{3}$ for simulated events generated both within the detector and outside the detector (in the dirt). The proportion of dirt events decreases closer to the center of the tank.

Monte Carlo template fits to the data were performed to determine the relative amount of dirt events in the NCE sample. The fits vary only the overall fraction of of dirt events in the simulation to best match the data in distributions of reconstructed radius $(R)$, volume $\left(R^{3}\right)$, and beam direction $(Z)$.

The original, pre-fit Monte Carlo predicts a $24 \%$ dirt background. After the fits, the distributions of $R$ and $R^{3}$ indicate that the predicted fraction of dirt events should be increased to $30 \%$ to better match the data. The spatial distribution of simulated events in $Z$ also does not agree with the data distribution, but in such a way that the fit to this variable suggested decreasing the background to $17 \%$.

The simplest resolution of these conflicting results is to continue to use the original 
Monte Carlo model to predict dirt events but apply a systematic error large enough to span the difference between the results of the $R$ and $Z$ fits (an error of 25\%). This results in a dirt background of $24 \pm 6 \%$ in the NCE sample. It is likely that future analyses will employ a more sophisticated method of dealing with this issue or perhaps modify the dirt simulations to better match the data.

However even with the current model, the dirt background can be reduced with cuts on the fiducial volume. Figure 5.6 shows the reconstructed $R^{3}$ distribution for events generated both within and outside the detector volume. As reconstructed radius decreases, the proportion of dirt events also decreases. Keeping only those events with reconstructed radius less than $400 \mathrm{~cm}$ reduces the overall dirt background to $16 \pm 4 \%$.

\subsection{Cuts Summary}

The selections outlined so far are the only ones applied to the data and Monte Carlo event samples for this analysis. They are summarized here for ease of reference. Table 5.2 lists the cuts, their purpose, and what they do to improve the neutral current elastic sample. Table 5.3 shows how the selections affect the neutral current elastic signal, and the neutrino backgrounds generated within the detector. Figure 5.7 illustrates the compositon of the NCE sample after all of the selections have been applied. There are 5562 total events in the sample of which 3680 are predicted to be signal.

\subsection{Neutrino Backgrounds}

After choosing these selections, the simulated sample is $84 \%$ pure neutral current elastic events. Of the remainder, roughly $1 \%$ is CCQE, $1 \%$ is a combination of CC resonant $1 \pi$, coherent $\pi$, and multi- $\pi$ production, and the remaining $14 \%$ is neutral current resonant 


\begin{tabular}{l|l|l}
\hline Selection & Purpose & Effectiveness \\
\hline Veto PMT Hits $<6$ & Remove cosmic rays & Of Monte Carlo $\nu_{\mu}$ events \\
1 Subevent & No decaying particles & simulated within the detector, \\
$4400<$ Time $(\mathrm{ns})<6500$ & Events in beam time & these four cuts isolate an $80 \%$ \\
Tank PMT Hits $<150$ & Ensures low energies & \multicolumn{1}{c}{ pure NCE sample. } \\
\hline Prompt Hits Fraction $<0.55$ & assures protons & $\begin{array}{l}\text { beam unrelated backgrounds } \\
\text { from } 31 \% \rightarrow 2 \% .\end{array}$ \\
Reconstructed $\mathrm{R}<400 \mathrm{~cm}$ & reduces dirt bkgds & $\begin{array}{l}\text { dirt backgrounds from } 24 \% \rightarrow \\
(16 \pm 4) \% \text { of data. }\end{array}$ \\
\hline
\end{tabular}

Table 5.2: Neutral current elastic sample selection.

\begin{tabular}{l|rr|rrrr}
\hline Selection & NCE evts & purity & NCE eff & CCQE eff & NC1 $\pi$ eff & Other eff \\
\hline No cuts & 27,400 & 0.18 & 1.00 & 1.00 & 1.00 & 1.00 \\
Veto PMTs $<6$ & 16,900 & 0.21 & 0.62 & 0.50 & 0.68 & 0.50 \\
1 Subevent & 14,200 & 0.50 & 0.52 & 0.08 & 0.49 & 0.07 \\
$4400<$ Time $<6500$ & 14,100 & 0.50 & 0.51 & 0.08 & 0.49 & 0.06 \\
Tank PMTs $<150$ & 12,800 & 0.82 & 0.47 & 0.01 & 0.17 & 0.01 \\
Prompt Frac $<0.55$ & 10,804 & 0.81 & 0.39 & $<0.01$ & 0.16 & $<0.01$ \\
Recon R $<400 \mathrm{~cm}$ & 4,229 & 0.84 & 0.15 & $<0.01$ & 0.05 & $<0.01$ \\
\hline
\end{tabular}

Table 5.3: Effect of data selections on NCE purity as well as signal and background efficiencies all determined from simulation. Column 1 indicates the predicted number of NCE events passing the cuts in a $6.57 \times 10^{19}$ POT sample. 


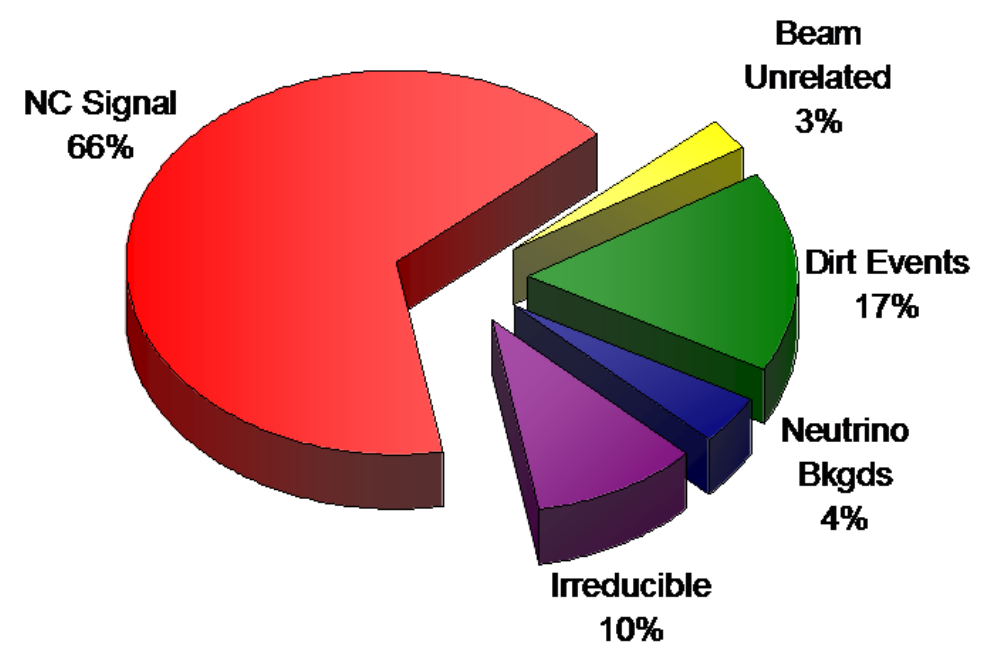

Figure 5.7: Composition of the full NCE data sample after all selections.

single pion production. Of the resonant $1 \pi$ background, Nuance predicts that the pion from the original neutrino interaction is absorbed in the nucleus $\sim 80 \%$ of the time (Chapter 4 ). Such events exactly resemble neutral current elastic events in the final state: one or more nucleons, and some soft photons.

The resonant single pion events that produce no pion in the final state are an irreducible background. There is no possible way to distinguish these from NCE events within the detector. It is therefore a philosophical decision whether to include such events as part of the signal or part of the background for further analysis. The approach taken here was to include these pion events as part of the background for the calculation of the neutral current differential cross section. Figure 5.8 shows the predicted contribution of this background relative the predicted NCE signal. This will be compared to the differential cross section determined for the data in the next Chapter. 


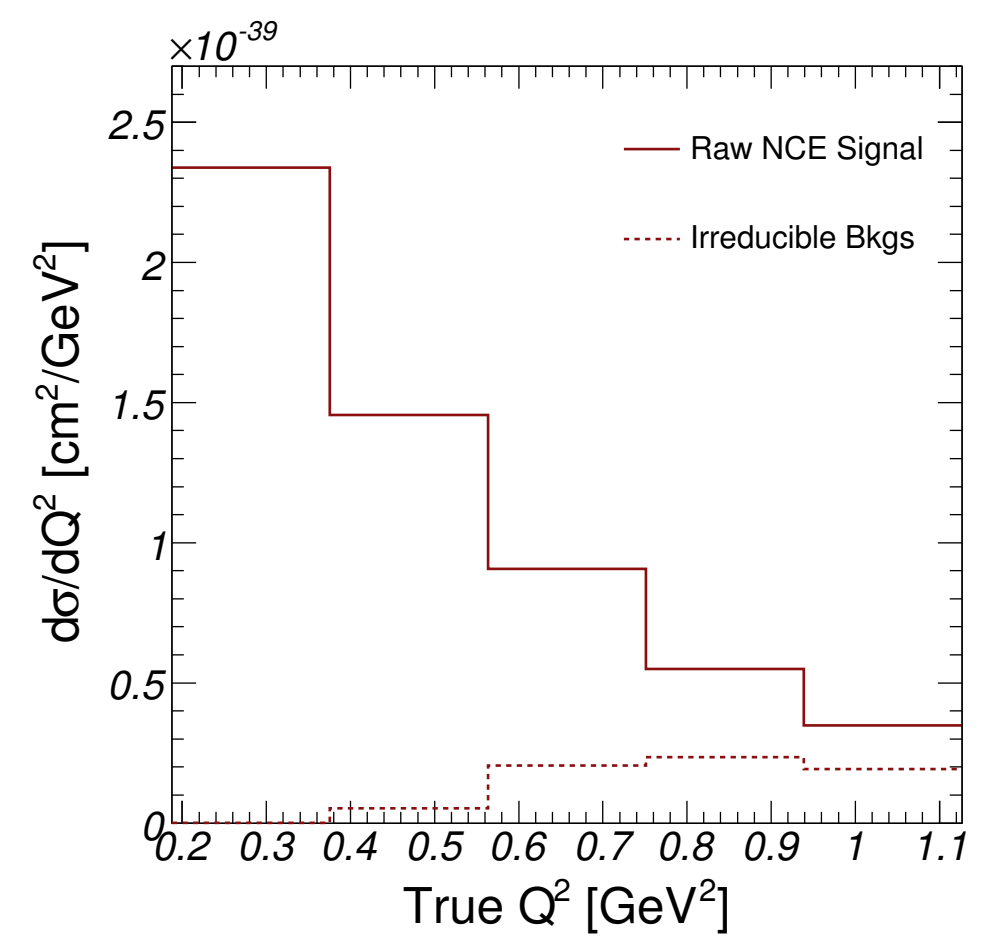

Figure 5.8: Monte Carlo predicted differential cross section for the irreducible backgrounds (dashed histo) and the neutral current elastic signal (solid histo). 


\section{Chapter 6}

\section{MiniBooNE NCE Results}

The goal of this analysis is to measure the neutral current elastic cross section as a function

of $Q^{2}$ and to extract the quasi-elastic axial mass $M_{A}^{Q E}$. This chapter describes the methods used and presents the results.

\subsection{Energy calibration}

In order to produce a neutral current elastic (NCE) differential cross section as a function of $Q^{2}, Q^{2}$ must be calibrated for NCE events from an observable quantity. Choosing this observable becomes straightforward using the result from Chapter 2 that $Q^{2}$ is proportional to the outgoing nucleon kinetic energy $\left(Q^{2}=2 m_{N} T_{N}\right)$ : kinetic energy is in general directly related to the total visible energy in an event. The total light, the total number of photons emitted in an event, is reconstructed by the Stancu Flux Fitter (Chapter 4) for both scintillation and Cherenkov photons. This analysis uses the sum of the scintillation and Cherenkov light fluxes to calibrate the kinetic energy and thus determine $Q^{2}$.

The calibration procedure is simple: the profile distribution of the total light flux vs true Nuance nucleon kinetic energy (figure 6.1) is fit to a second order polynomial. The fit 

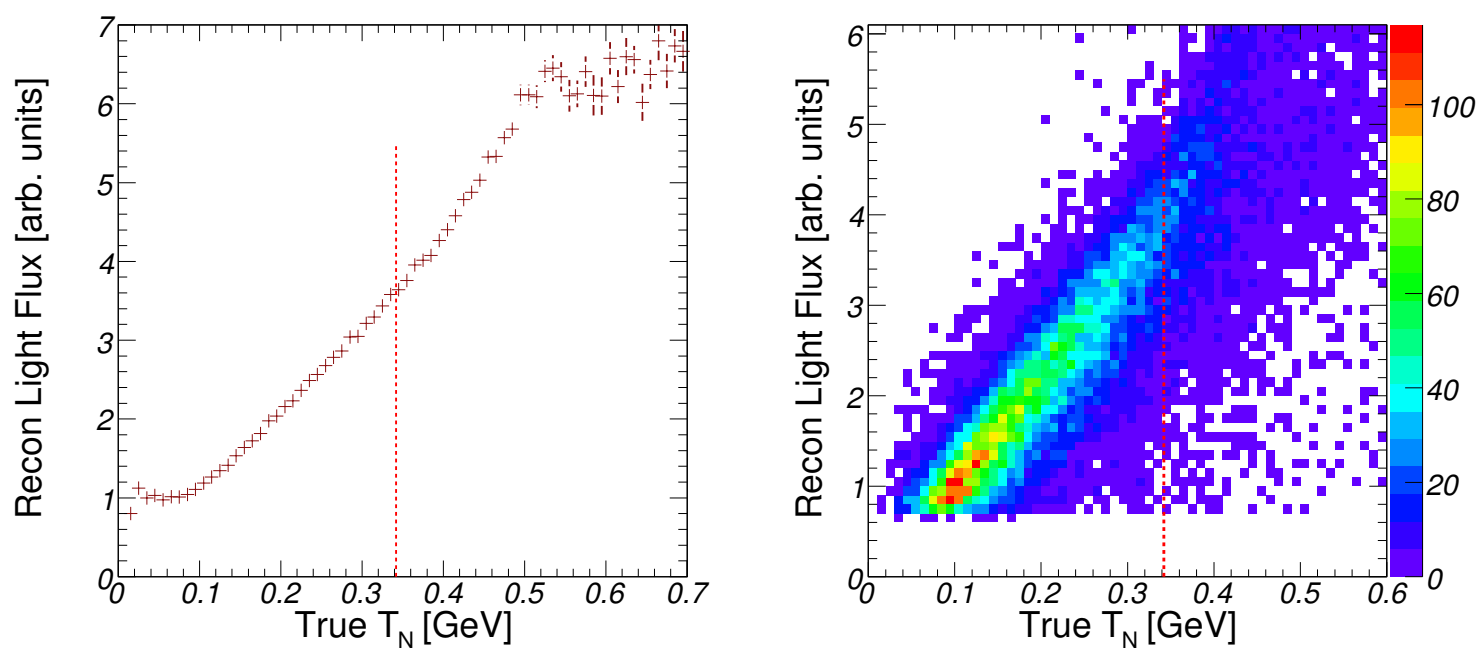

Figure 6.1: Nucleon kinetic energy calibration for Monte Carlo events. Left panel: profile histogram used to fit the light flux to the true nucleon kinetic energy. Right panel: the amount that the flux is smeared as a function of true energy. The dashed lines indicate Cherenkov threshold.

is performed in a low flux region (flux: $1 \rightarrow 5$ ) and a high flux region (flux: $5 \rightarrow 6.5$ ) with the results constrained to match at the interface. The results of the calibration are shown in figure 6.2 .

Comparing the Monte Carlo fit results to the data can be performed using proton Cherenkov threshold as a point of reference. Until threshold, light emmitted in neutral current interactions should be composed almost entirely of scintillation photons. After Cherenkov threshold, the proportion of scintillation light should decrease as these interactions also begin to produce Cherenkov radiation. Figure 6.3 shows how this transition looks in MiniBooNE.

The figure shows the fraction of scintillation light as a function of energy. At low kinetic energy, protons do not emit as strongly, and a fraction of radiation comes from sources of prompt light such as nuclear de-excitation photons (Chapter 4). As protons increase in 


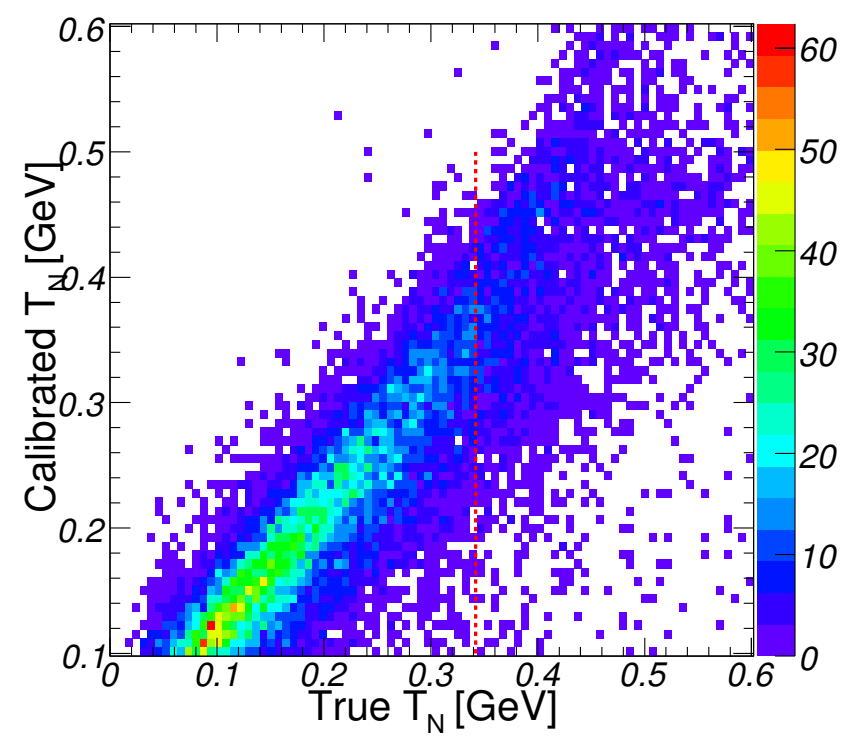

Figure 6.2: Calibrated kinetic energy versus true kinetic energy (Monte Carlo).
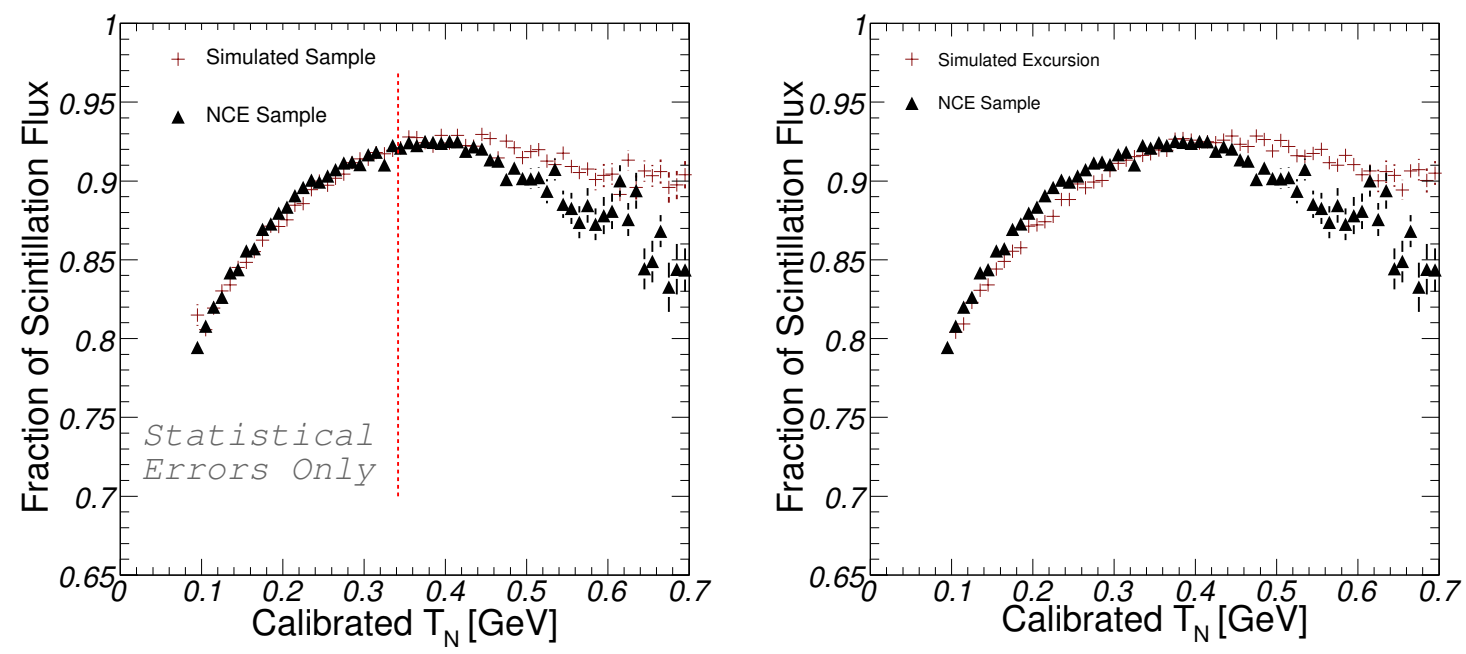

Figure 6.3: Cherenkov threshold transition for NCE events. Left panel: NCE data in good agreement with Monte Carlo in the energy range of interest. Right panel: a $7 \%$ positive excursion in the calibration. The agreement is now visibly worse in the $\sim 100-300 \mathrm{MeV}$ energy range. 


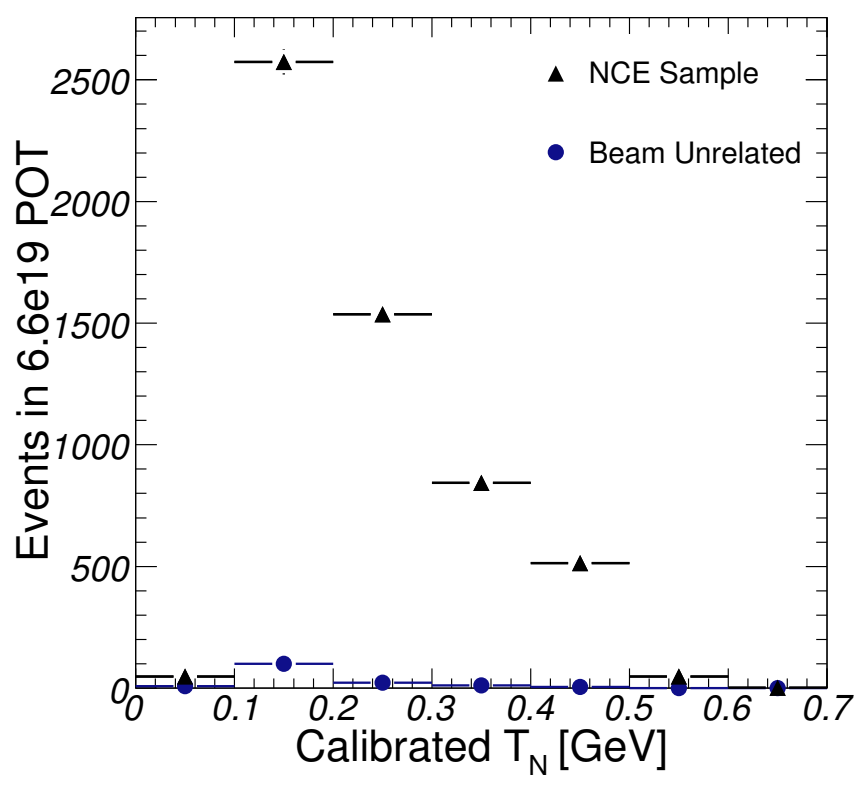

Figure 6.4: Calibrated kinetic energy for beam events passing NCE cuts shown with the beam unrelated background.

energy, they emit more photons and raise the fraction of scintillation light. However once protons reach Cherenkov threshold (indicated by the dashed line), the distribution turns over again. Since the shape and energy at which the scintillation fraction turns over are the same for data and Monte Carlo, the same calibration can be used to generate calibrated kinetic energy distributions for both. A systematic error is applied to the calibrated energy by varying the calibration function until the resultant kinetic energy shifts by a noticeable amount.

Figure 6.4 shows neutrino events passing NCE cuts along with the beam unrelated activity. The unrelated background distribution has been normalized to $0.35 \pm 0.35$ according to the methods outlined in Chapter 5 . This small background is subtracted from the data in all further distributions because it is not included in the simulations. 

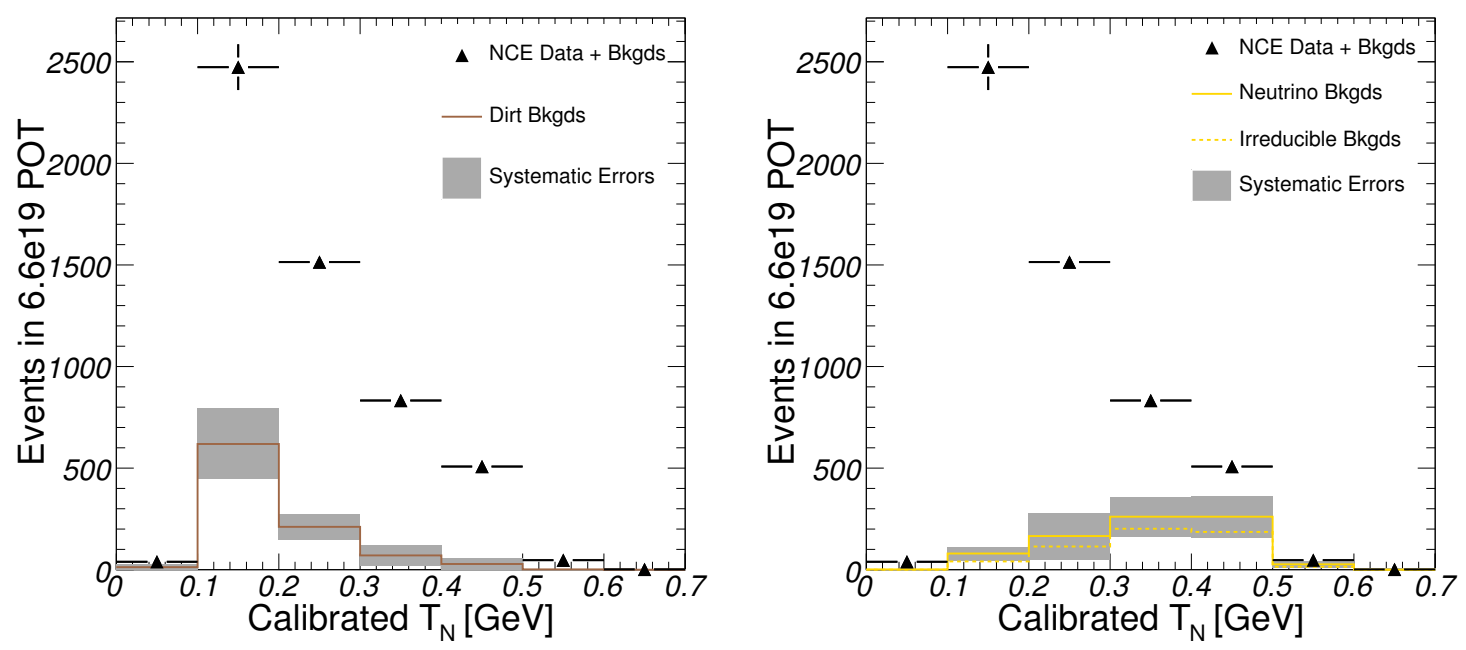

Figure 6.5: Calibrated kinetic energy for strobe subtracted NCE data shown with simulated backgrounds. Left panel: dirt background with dirt systematic errors. Right panel: neutrino backgrounds with relevant errors. The dashed line indicates the size of the irreducible component of those backgrounds.

Figure 6.5 shows the size and energy distribution of predicted backgrounds with systematic errors. These backgrounds are normalized to have the same protons on target (POT) as the data. The dirt background, like the data, peaks at low energies, whereas the neutrino (NC pion mis-ID) backgrounds increase at higher energies until they are removed by the THits cut. The irreducible contribution to the neutrino background (Chapter 5) is indicated by the dashed histogram.

Figure 6.6 compares data and Monte Carlo events passing the NCE selections. That the data is consistently lower than the Monte Carlo may indicate a different $M_{A}^{Q E}$ than predicted (section 6.3) or some other physics. The total ratio of data to Monte Carlo in this energy range is

$$
R\left(T_{N}=0.0 \rightarrow 0.7 \mathrm{GeV}\right)=0.92 \pm 0.04(\text { stat }) \pm 0.20(\text { syst })
$$




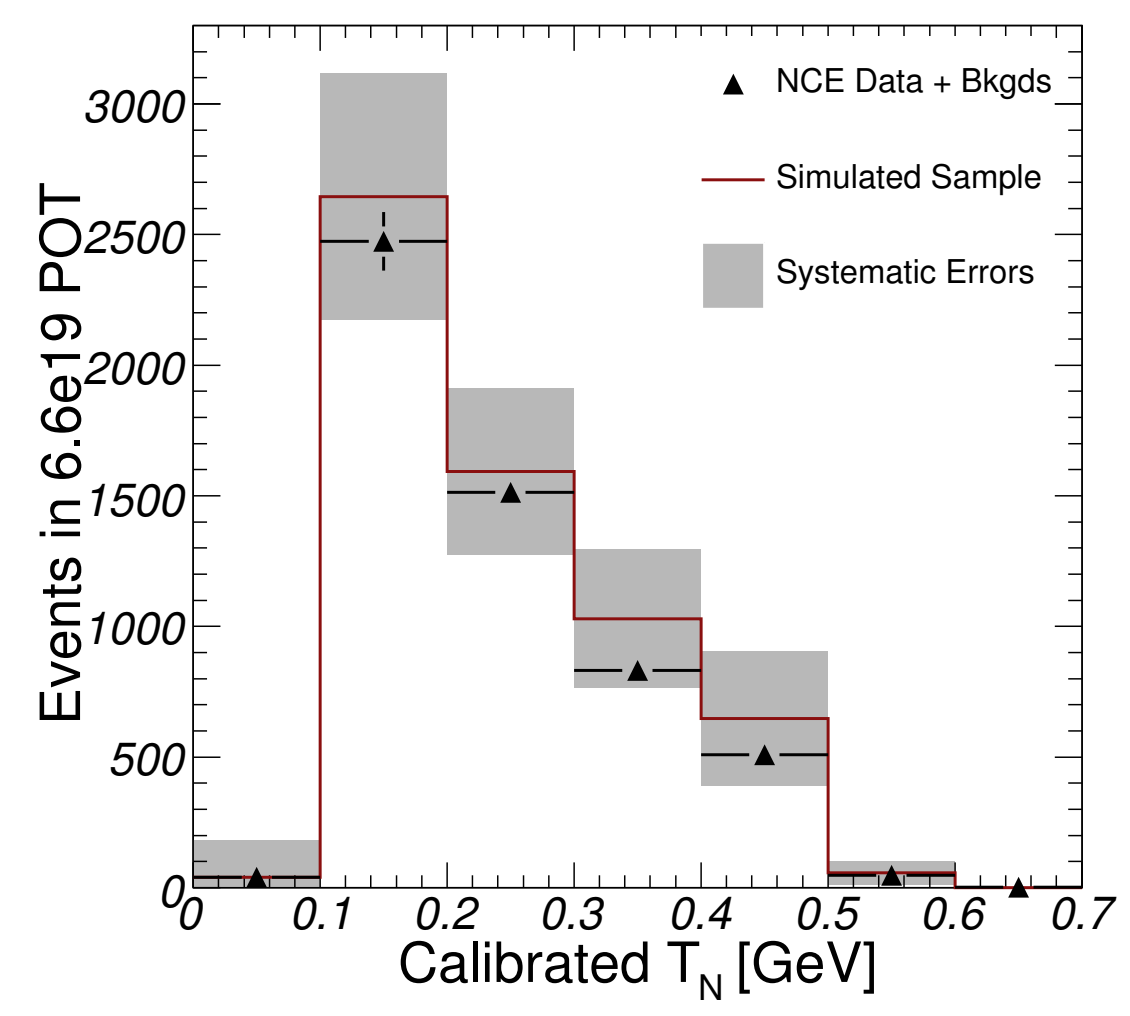

Figure 6.6: Calibrated kinetic energy for strobe subtracted NCE data together with prediction. The data and simulations agree within the systematic error bands shown. 


\subsection{Error Propagation}

The error bands in figure 6.6 represent the uncertainties on the simulation models. The particular sources of error were discussed in Chapter 4. This section explains the methods that determine the size of these errors.

\subsubsection{Multisims}

In general, MiniBooNE measures its model uncertainties by varying parameters within their errors to see how those changes affect the final simulated results. When parameters in a model are correlated, all of them must be modified simultaneously to determine both the effects of each parameter alone and also of the correlations between parameters. Error simulations where multiple parameters are tuned simultaneously are called "multisims" in MiniBooNE.

In some situations, the result of the model is defined by a function of the parameters. For example, the $\pi^{+}$production cross section is a Sanford-Wang parameterization of nine parameters $c_{1}, \ldots, c_{9}$ (Chapter 4). In these cases, changing one or more parameters is equivalent to multiplying the function with the original "central value" parameters by a weight. Thus to understand how uncertainties in the parameters affect the results, weights can be generated for an event from each model and then applied to that event in any distribution. Uncertainties in the beam, flux $\left(K^{0}, K^{+}, \pi^{-}\right.$, and $\pi^{+}$production), and cross section models can all be treated with this scheme of weighted multisims. The results for these models are displayed in figure 6.7.

The detector light propagation model cannot be treated with the method of weighted multisims. Though it has many correlated parameters, the effects of those parameters on event topology are not always understood in terms of a function (i.e. it cannot be written in a functional form with variations parameterized with weights). In that case, the most 

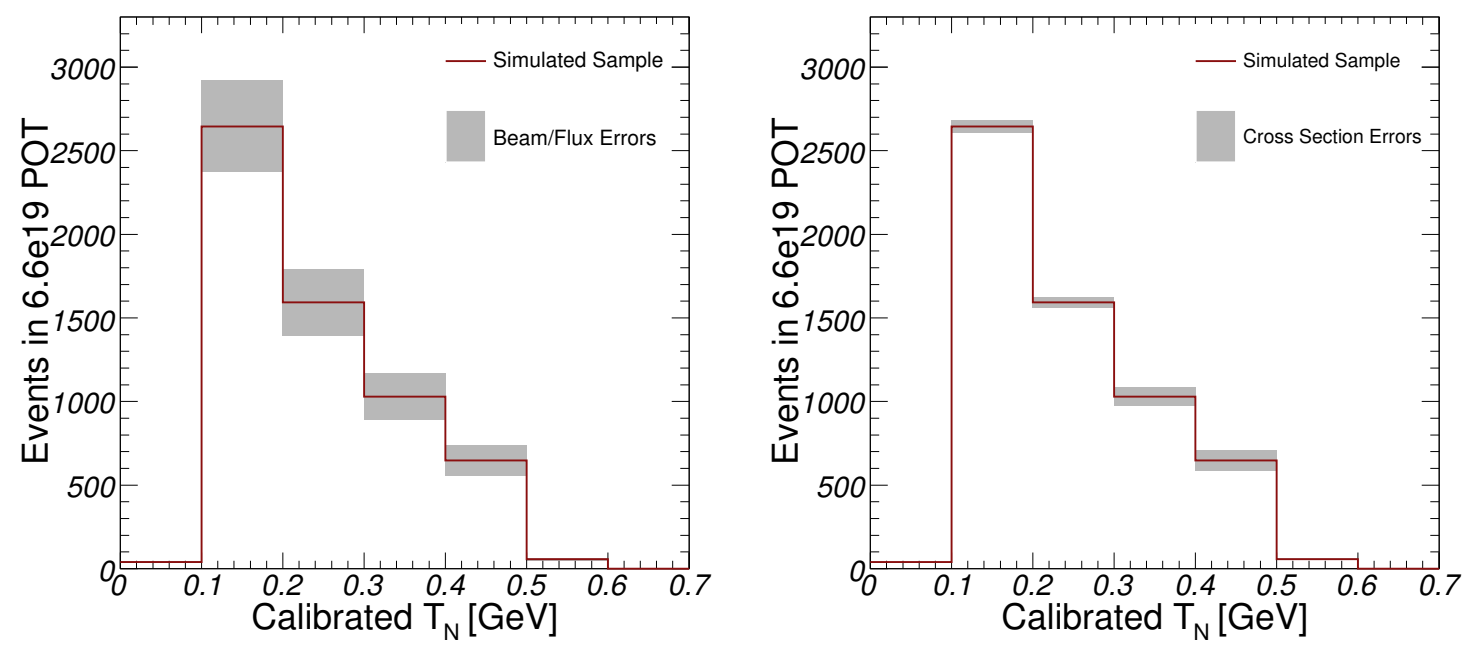

Figure 6.7: Beam/Flux and cross section systematic errors (Monte Carlo).

effective way to determine errors on observed quantities is to modify all the input parameters simultaneously and re-simulate events. To sample as much of parameter space as possible, $\sim 50$ different re-simulations were generated. The left panel of figure 6.8 shows the results of the light propagation model multisims.

\subsubsection{Unisims}

In some cases, when a parameter is not correlated to anything else, a single $1 \sigma$ variation (a "unisim") can be generated to determine the error on the final result. Uncertainties from the choice of hadronic model (GCALOR vs GFLUKA), phototube discriminator threshold, and charge-time correlations were treated with unisims. These are shown in the right panel of figure 6.8. In addition, uncertainties specific to the neutral current elastic analysis were assessed this way: the kinetic energy calibration, normalization of the dirt background, and prediction of the dirt background energy shape. The results of these are shown in figure 6.9. 

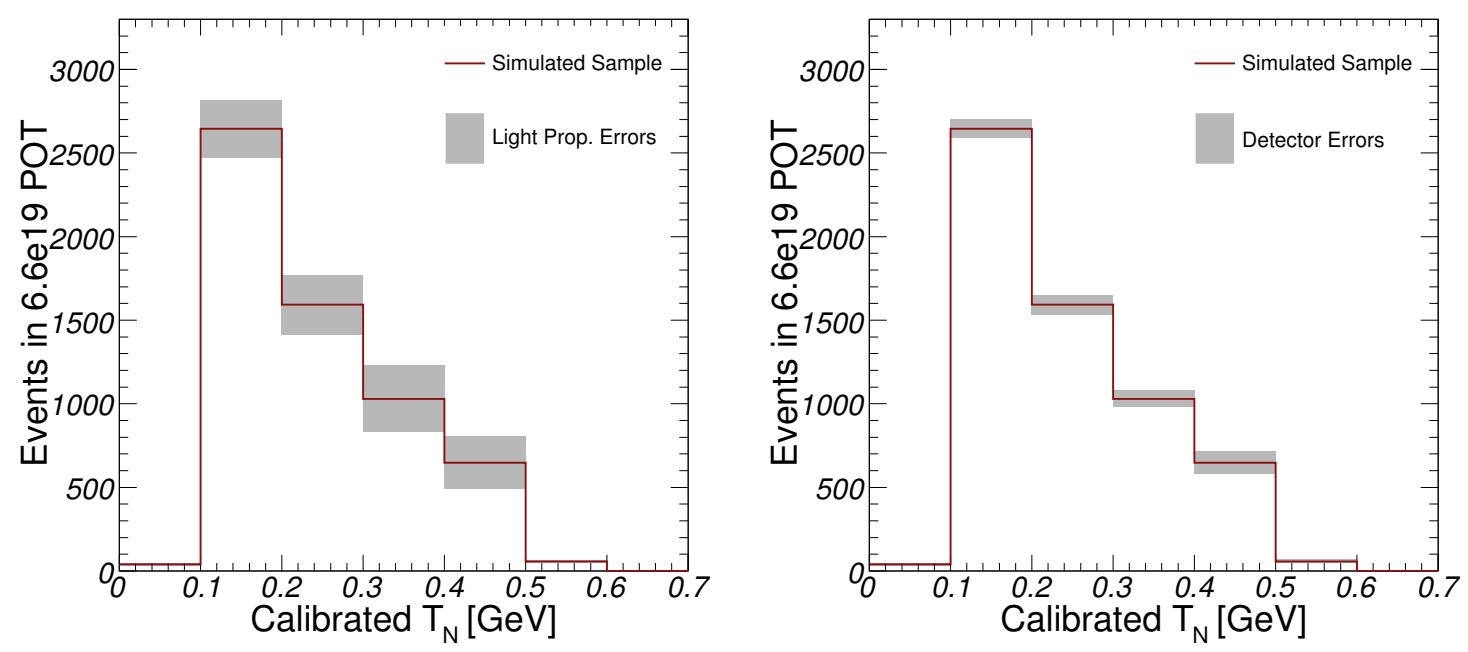

Figure 6.8: The systematic error contributions from the light propagation multisims and the detector model unisims (Monte Carlo).
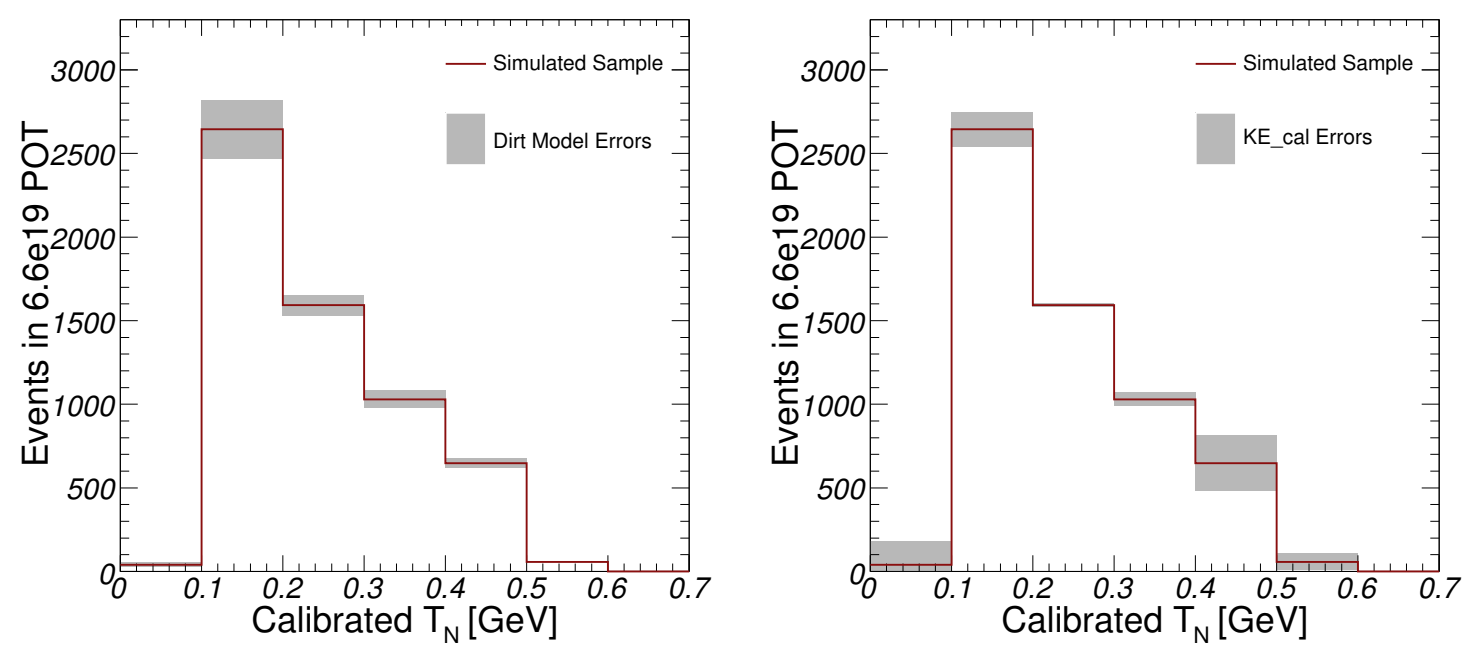

Figure 6.9: Dirt model and kinetic energy calibration errors (Monte Carlo). 


\subsubsection{Error Matrices}

Each set of multisims and each unisim is used to produce an error matrix. If $\eta$ simulations were generated for a particular set of variations $\alpha$ (one of the multisims or unisims discussed in the previous subsection), the elements of the error matrix are

$$
m(\alpha)_{i j}=\frac{1}{\eta-1} \sum_{k=1}^{\eta} \frac{\left(N_{i}^{k}(\alpha)-N_{i}^{0}\right)\left(N_{j}^{k}(\alpha)-N_{j}^{0}\right)}{\left(N_{i}^{0} N_{j}^{0}\right)}
$$

where $N_{i}^{k}(\alpha)$ is the number of events in the $i$ th bin of the $k$ th simulation of the $\alpha$ multi/unisim, and $N_{i}^{0}$ is the number of events in the $i$ th bin of the un-varied "central value" Monte Carlo [131, 132]. In the case of unisims where $\eta=1$, the normalization outside the sum is set to 1. This formula generates relative error matrices that can be normalized to a distribution with any number of events.

Error matrices from different multi or unisims are assumed to have no correlations. Therefore the separate relative error matrices are added together to determine the complete uncertainty on kinetic energy. This full error matrix $m_{i j}$ shown in figure 6.10 is also used in $\chi^{2}$ calculations to determine parameters such as $M_{A}^{Q E}$.

\subsection{Determining $M_{A}$ from Nucleon Kinetic Energy}

Although further processing of the calibrated kinetic energy distributions is necessary to determine the NCE cross section, they can be used at this stage to extract $M_{A}^{Q E}$. The shape of the data kinetic energy distribution is compared to simulated distributions in which $M_{A}^{Q E}$ and $\Delta \mathrm{s}$ have been varied. A $\chi^{2}$ is calculated for each simulation, and the lowest $\chi^{2}$ determines the best $M_{A}^{Q E}$ value.

For this analysis, $M_{A}^{Q E}$ and $\Delta \mathrm{s}$ were varied in a $50 \times 50$ grid for $1.02 \leq M_{A} \leq 2.00 \mathrm{GeV}$ and $-0.48 \leq \Delta \mathrm{s} \leq 0.50$. The method of weighted multisims (section 6.2) was used to make the 2500 kinetic energy distributions for this analysis. 


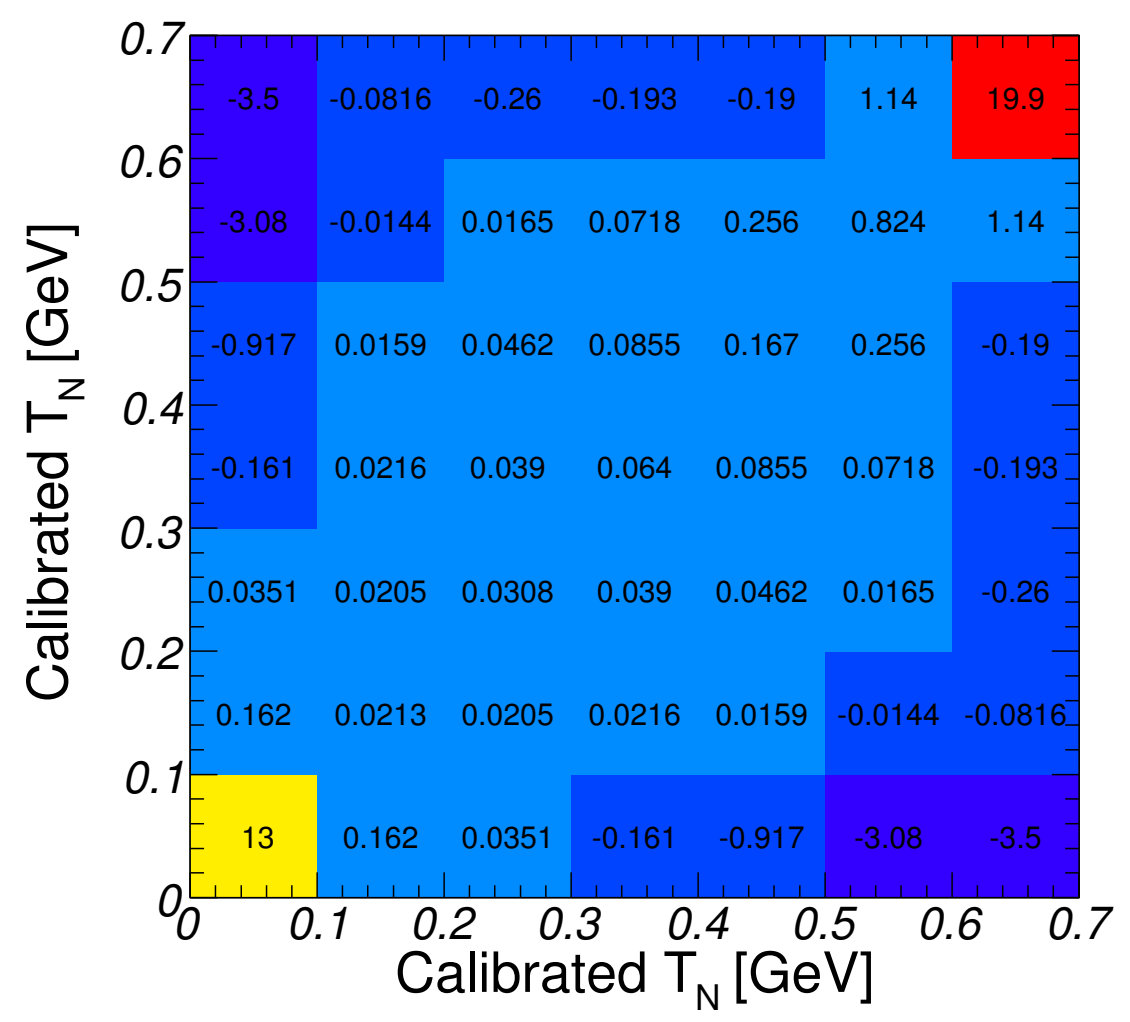

Figure 6.10: The complete error matrix. This is the sum of all the multi/unsim error matrices described here and used in this analysis (Monte Carlo). 

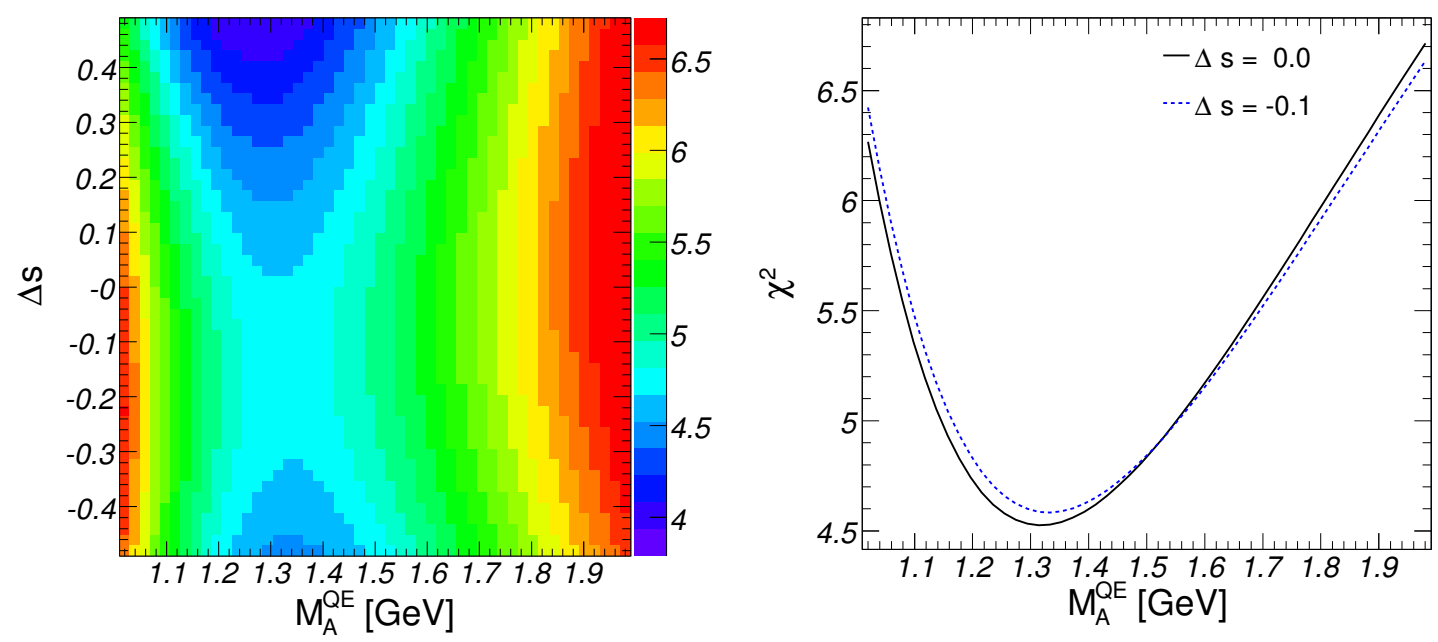

Figure 6.11: $\Delta \mathrm{s} M_{A} \chi^{2}$ parameter space and $\chi^{2}$ parabola for $M_{A}$ (Monte Carlo).

The $\chi^{2}$ for each grid point $\kappa$ is calculated in the standard way for the full error matrix:

$$
\chi^{2}(\kappa)=\sum_{i}^{b} \sum_{j}^{b}\left(N_{i}^{0}-N_{i}^{\kappa}\right)\left(N_{i}^{0} m_{i j} N_{j}^{0}\right)^{-1}\left(N_{j}^{0}-N_{j}^{\kappa}\right)
$$

where $b$ is the number of calibrated kinetic energy bins, $N_{i}^{0}$ is the number of events in the $i$ th data bin, $N_{i}^{\kappa}$ is the number of events in the $i$ th bin of the simulation for grid point $\kappa$, $\left(N_{i}^{0} m_{i j}^{-1} N_{j}^{0}\right)^{-1}$ is the inverse of the data-normalized error matrix, and the simulated kinetic energy distributions are normalized to have the same integral as the data distribution (i.e. the normalization is fixed). Although seven bins are shown in plots of kinetic energy, only the central five $\left(T_{N}=0.1 \rightarrow 0.6 \mathrm{GeV}\right)$ are used to construct the $\chi^{2}$. The outlying bins have low efficiency, which can cause undesirable effects in a matrix inversion. This will be discussed further in section 6.4

Figure 6.11 displays the resultant $\chi^{2}$ distribution. The range of $\chi^{2}$ values is reasonable considering that there are 3 degrees of freedom ( 5 bins - 2 parameters). This suggests that the size of the errors in the matrix are also reasonable (not over or under estimated). Moreover, these errors are large enough that they do not provide a tight constraint of $M_{A}^{Q E}$. 
The best fit value of $M_{A}^{Q E}$ for $\Delta s=0.0$ is $1.34_{-0.24}^{+0.38} \mathrm{GeV}$ as determined from the plot on the right panel of figure 6.11. This result agrees within errors with the predicted result from the MiniBooNE CCQE analysis: $M_{A}^{Q E}=1.23 \pm 0.20 \mathrm{GeV}$. It is also in reasonable agreement with the results from BNL 734: $M_{A}^{Q E}=1.06 \pm 0.05 \mathrm{GeV}$ [40]. The $M_{A}^{Q E} \chi^{2}$ values for $\Delta s=-0.1$ (the value predicted by DIS experiments) is also displayed and found to be nearly identical: $M_{A}^{Q E}=1.36_{-0.24}^{+0.40}$.

In the $\chi^{2}$ map of figure 6.11, it naively appears that extremely high or extremely low values of $\Delta s$ are prefered by the fit. However as described in Chapter 2, current experimental evidence puts $\Delta s$ at approximately -0.1 or 0.0 with small errors. Values of +0.4 or -0.4 are not physically meaningful and have been included here only to provide context for the regions that are of interest. The large excursions in $\Delta s$ affect the shape of the distribution in a way that is preferred by the minimization. But if that shape is indeed better, it must be explained by modification of other physical parameters in the simulations.

In reality, this $\chi^{2}$ map (left panel of figure 6.11) explicitly illustrates the expectation that there is no sensitivity to $\Delta s$ with this analysis of NCE events. The mechanisms by which neutrons interact mimic proton events to such a degree that they cannot be distinguished. And although there are $33 \%$ more proton than neutron targets in the mineral oil, the cross section for neutrons is about $40 \%$ higher. Since the sign of the axial form factor in the NCE cross section is opposite for proton and neutron scattering, this averages-out the measured value of $\Delta s$.

Figure 6.12 shows the data with two different values of $M_{A}^{Q E}$ for $\Delta \mathrm{s}=0$. The left panel is with the best fit $M_{A}^{Q E}$ from this analysis. The right panel is with the best fit $M_{A}^{Q E}$ from the BNL 734 analysis. Although the Monte Carlo distribution in the right panel naively seems like the better fit to the data, correlations in the error matrix pull $M_{A}^{Q E}$ to the higher best fit value for this study. 

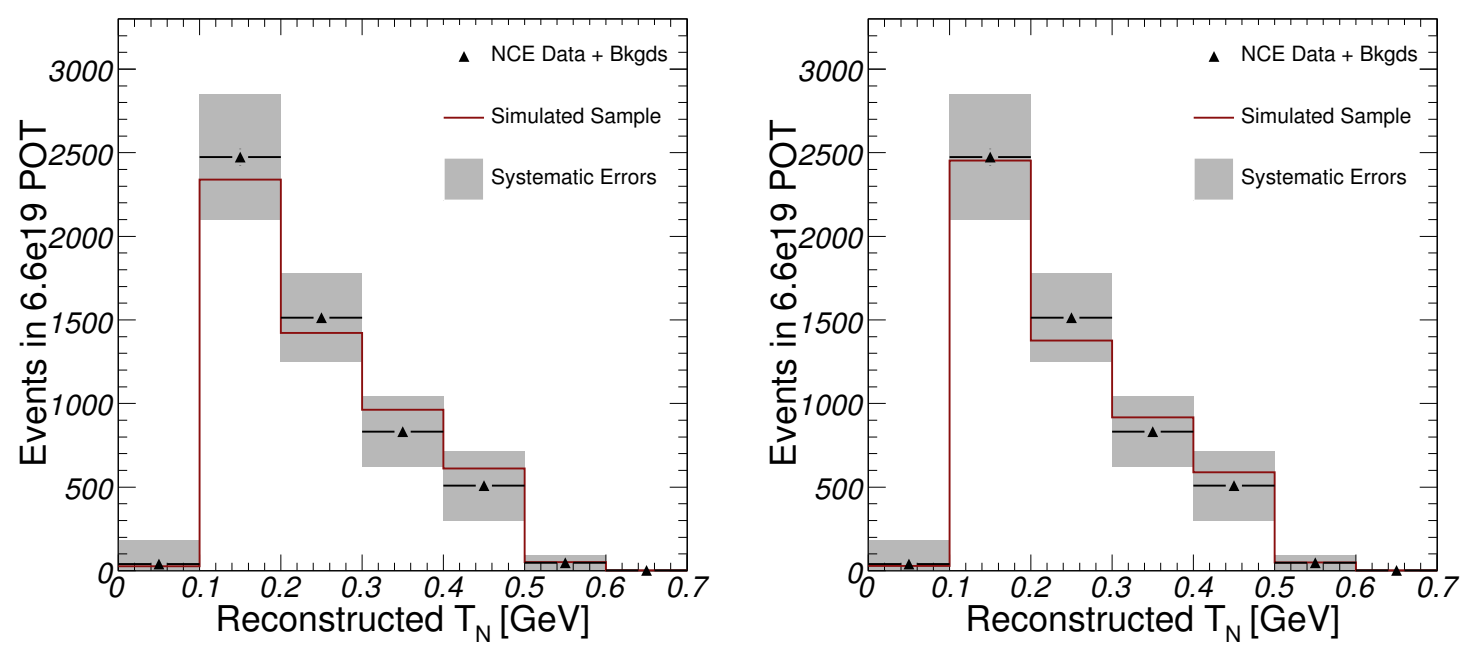

Figure 6.12: Left panel: MiniBooNE data with $M_{A}^{Q E}=1.34 \mathrm{GeV}$. Right panel: MiniBooNE data with the BNL 734 best value of $M_{A}^{Q E}=1.06 \mathrm{GeV}$. In both, $\Delta \mathrm{s}=0$.

\subsection{Unfolding The Differential Cross Section}

To calculate a differential cross section that can be compared to other experiments and to theory, the calibrated kinetic energy must be made detector and model independent through a process called unfolding.

The measured data events contain detector effects (resolution, etc.) that distort the true physics. Although these are also modeled by the detector Monte Carlo, that part of the simulation can also be turned off in order to study events with only the Nuance nuclear model predictions.

The simulated pre- and post- detector Monte Carlo events can be used to form an efficiency matrix: the probability of measuring $y$ for the kinetic energy of an event when it truly occurred with kinetic energy $x$ (figure 6.13). The efficiency matrix describes how to 


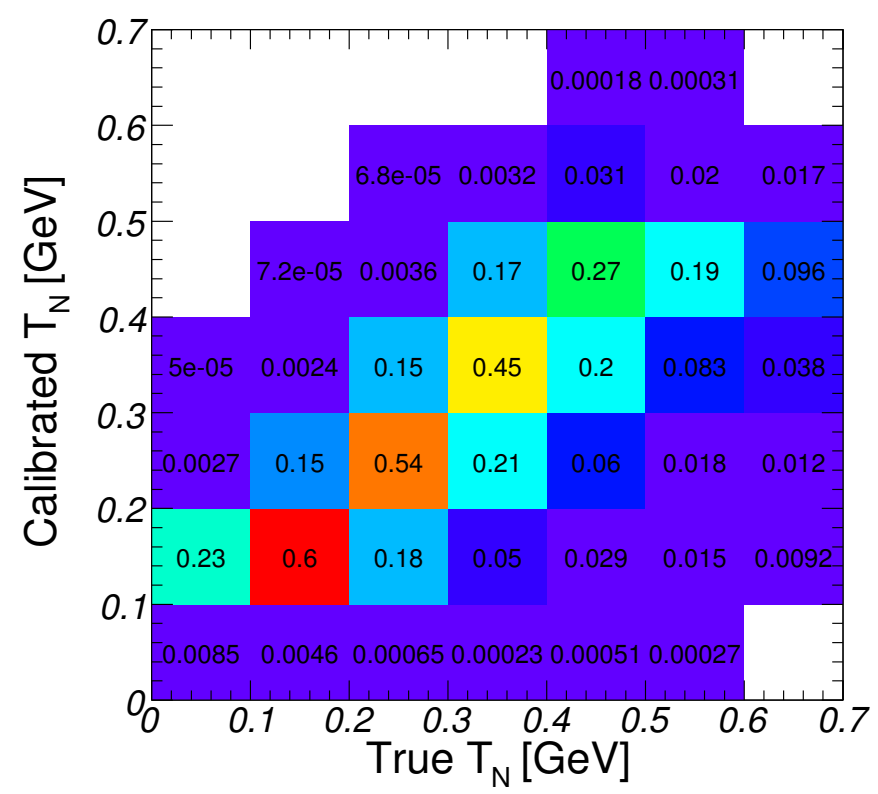

Figure 6.13: Efficiency matrix from the Monte Carlo for the NCE analysis.

turn the original physics into what the detector observes:

$$
T_{i, \text { calibrated }}=\sum_{j} \epsilon_{i j} T_{i, p h y s i c s}
$$

where $\vec{T}_{\text {calibrated }}$ is the observed (calibrated) kinetic energy distribution, $\vec{T}_{\text {physics }}$ is the real physics kinetic energy distribution, and $\epsilon$ is the efficiency matrix.

The standard method for unfolding is to multiply the calibrated kinetic energy by the inverse of the efficiency matrix

$$
T_{i, p h y s i c s}=\sum_{j} \epsilon_{i j}^{-1} T_{i, c a l i b r a t e d}
$$

Unfortunately, the considerable smearing in the NCE efficiency matrix corrupts this procedure. Instead of recovering the original structure that was present in the true distribution, a highly smeared matrix "recovers" statistical fluctuations in the data distribution [133]. 
Many techniques exist to unfold data despite unstable efficiency matrices. The technique used in this analysis is to unfold the data with a correction factor $\vec{C}$

$$
T_{i, \text { physics }}^{\text {data }}=C_{i} T_{i, \text { calibrated }}^{\text {data }}
$$

where $\vec{C}$ is calculated from the Monte Carlo

$$
C_{i}=T_{i, \text { predicted }}^{M C} / T_{i, \text { calibrated }}^{M C}
$$

and the elements of the denominator can be rewritten in terms of the numerator and the efficiency matrix $\epsilon$

$$
T_{i \text { calibrated }}^{M C}=\sum_{j} \epsilon_{i j} T_{j \text { predicted }}^{M C}
$$

The inverse of the correction factor describes the efficiency for detecting events as a function of calibrated energy. Defining $\vec{\epsilon}^{\prime} \equiv 1 / \vec{C}$ allows the unfolded kinetic energy distribution to be written in terms of the calibrated distribution in the standard form of equation 6.5

$$
T_{i, \text { physics }}^{\text {data }}=\frac{1}{\epsilon_{i}^{\prime}} T_{i, \text { acalibrated }}^{\text {data }}
$$

If the efficiency matrix had only diagonal terms, this procedure would be equivalent to inverting the matrix. However, the off-diagonal elements present correct for the detector smearing.

The left panel of figure 6.14 shows the efficiency $\vec{\epsilon}^{\prime}$ for all neutral current elastic events. The right panel of that figure separates the efficiency into neutral current proton and neutron scattering events. The efficiencies for protons and neutrons are similar confirming that analyzing the combined neutral current cross section is reasonable.

Unfolding with a correction factor in this way incurs a bias if the energy shapes of the Monte Carlo and data differ

$$
\text { bias }_{i}=\left(\frac{T_{i \text { pred }}^{M C}}{T_{i \text { recon }}^{M C}}-\frac{T_{i \text { physics }}^{\text {data }}}{T_{i \text { recon }}^{\text {data }}}\right) T_{i \text { recon }}^{\text {data }} .
$$



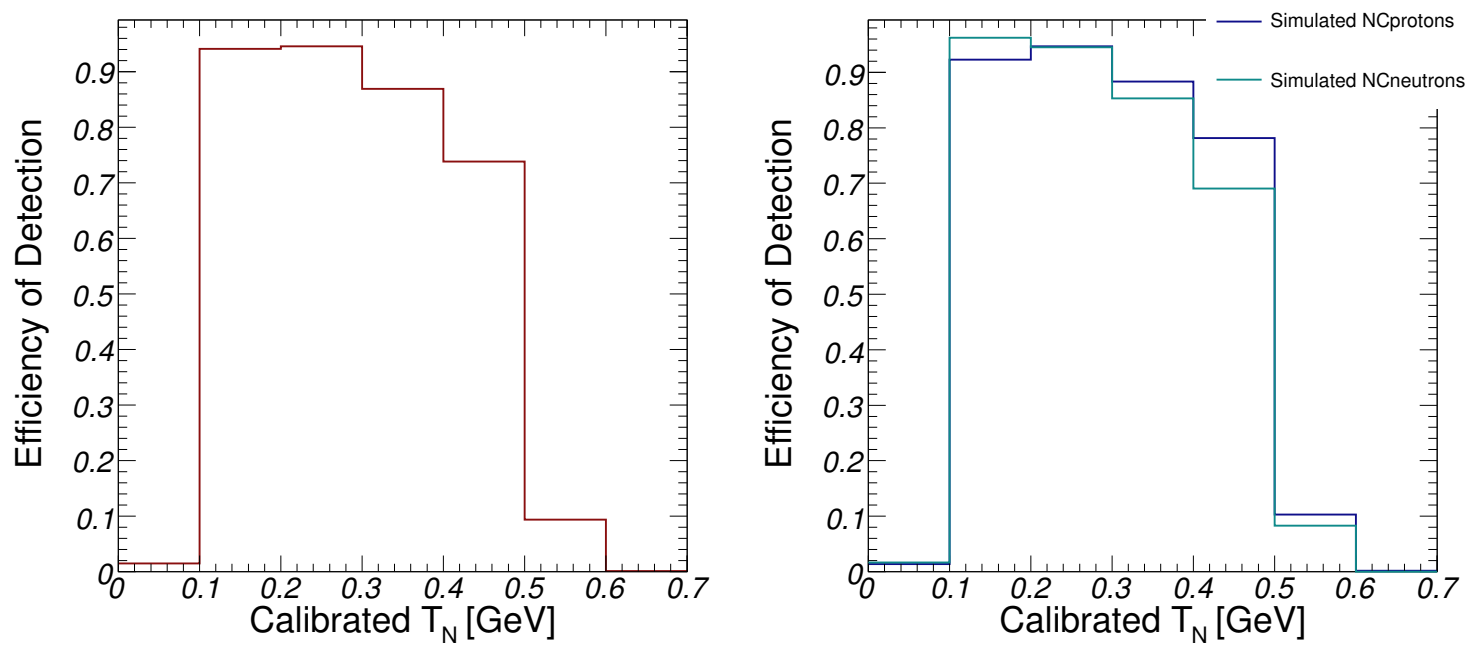

Figure 6.14: The efficiency for detecting all NCE events and the efficiency separated into proton and neutron scattering events (Monte Carlo).

However, this bias can be reduced by correcting the shape of the Monte Carlo to be more like the data. In practice, this is performed by iterating the unfolding procedure a single time. Tests using fake data samples indicate that the bias is both small and within errors, and therefore such a correction is reasonable.

This unfolding procedure is executed with all 7 kinetic energy bins (from 0.0 to $0.7 \mathrm{GeV}$ ). However, only the central 5 bins (from 0.1 to $0.6 \mathrm{GeV}$ ) are used for post-unfolding analysis. The low efficiencies in the outside bins (figure 6.14 means that very little can be determined about the cross section in those regions. However, keeping them through this stage of analysis allows events to feed into and out from them during unfolding in a natural way that makes the results of the retained bins more stable and accurate.

It is important to note that all of the kinetic energy distributions are unfolded: the data, the Monte Carlo prediction, and all of the simulated variations. Unfolding the variations is the most straightforward way of propagating uncertainties to the cross section. Doing so allows cross section errors to then be calculated using the method same described in 
section 6.2 ,

\subsection{The Neutral Current Elastic Cross Section}

After it has been unfolded, the resultant kinetic energy distribution must be transformed into a cross section:

$$
\frac{d \sigma_{i}^{N C E}}{d Q^{2}}=\frac{N_{i}^{N C E} /\left(d Q^{2} / d T_{N} \Delta T_{N}\right)}{N_{P O T} \int \Phi_{\nu} d E_{\nu} N_{N}}
$$

where $\frac{d \sigma_{i}^{N C E}}{d Q^{2}}$ is the cross section in the $i$ th unfolded kinetic energy bin. In the numerator $N_{i}^{N C E}$ is the number of NCE signal events in the $i$ th bin (the result of unfolding), the factor $\left.d Q^{2} / d T_{N} \Delta T_{N}=2 m_{N} * 0.1\right)$ accounts for the bin width of the unfolded $T_{N}$ distribution and the transformation of $T_{N}$ to $Q^{2}, m_{N}=0.939 \mathrm{GeV}$ (the average of the proton and neutron mass). The denominator contains the physics factors that scale the number of events into a cross section: the number of protons on target that generated the sample $N_{P O T}$, the integrated flux $\int \Phi_{\nu} d E_{\nu}$, and the number of nucleons $N_{N} \cdot N_{P O T}$ is measured experimentally [124] to be $(6.57 \pm 0.05) \times 10^{19}$. The integrated flux is determined by Nuance to be $5.167 \times 10^{-10}$ with errors determined from the multisims. The number of nucleons is calculated using $N_{N}=N_{A} \rho_{\text {oil }}\left(\frac{4}{3} \pi R^{3}\right)$ where $N_{A}$ is Avagadro's number, $\rho_{\text {oil }}=$ $0.86 \pm 0.01 \mathrm{~g} / \mathrm{cm}^{3}$ is the density of MiniBooNE oil, and $R=400 \mathrm{~cm}$ is the radius of the fiducial volume used for this analysis with errors determined by the multisims. The uncertainties in $N_{P O T}$ and $\rho_{\text {oil }}$ are each $\sim 1 \%$, much smaller than the uncertainties already applied to the unfolded kinetic energy distribution from multi and unisims. Including such small errors would not change the results, and therefore they are not included in the reported differential and inclusive cross sections.

The resulting unfolded differential cross section for the data with errors is shown in figure 6.15. The integrated cross section for $Q^{2}$ from 0.189 to $1.13 \mathrm{GeV}$ is

$$
\sigma\left(Q^{2}=.189 \rightarrow 1.13 \mathrm{GeV}^{2}\right)=(8.8 \pm 0.6(\text { stat }) \pm 2.0(\text { syst })) \times 10^{-40} \mathrm{~cm}^{2} .
$$




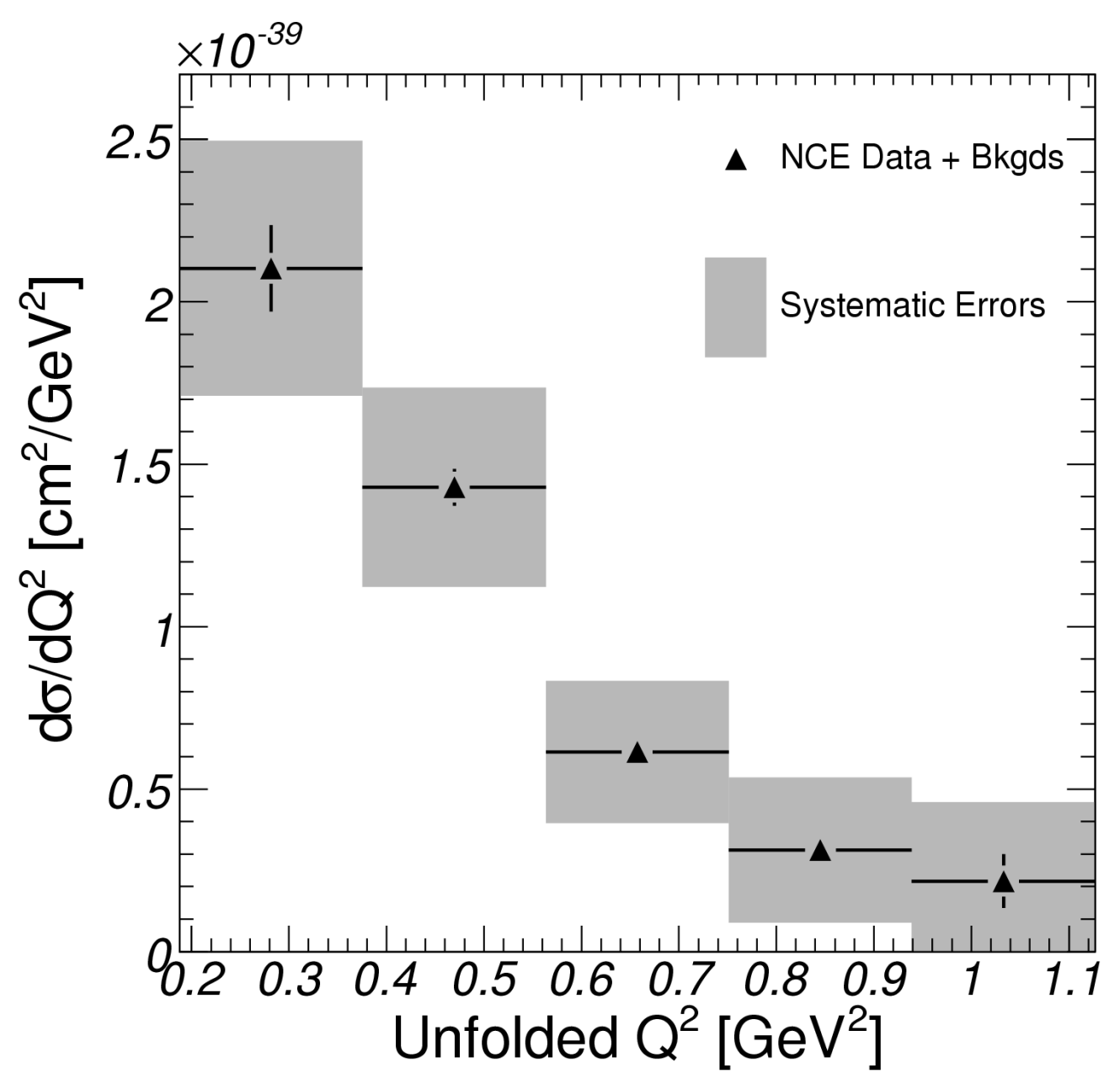

Figure 6.15: NCE data unfolded cross section with errors. 
Figure 6.16 compares the data result to the raw neutral current cross section predicted by the simulation as well as the cross section for the irreducible background (Chapter 5) subtracted from the data before unfolding.

Figure 6.17 compares the MiniBooNE neutrino neutral current elastic cross section to the BNL 734 neutrino proton elastic cross section (discussed in Chapter 2). It is interesting to observe that they seem to be in reasonable agreement, however it is important to note that the average MiniBooNE neutrino energy is $\left\langle E_{\nu}\right\rangle=0.8 \mathrm{GeV}$, and for BNL the average energy was $\left\langle E_{\nu}\right\rangle=1.2 \mathrm{GeV}$ with a long high energy tail. Although the ratio of the NCE cross section at these energies is nearly $1.0(\sim 0.96)$, still the most correct way to compare the two experiments would be to simulate the BNL neutrino flux in the MiniBooNE detector and to reconstruct and unfold the resulting events. Such a comparison is left for future work.

\subsection{Summary and Conclusions}

The current work on neutral current elastic scattering in MiniBooNE, an analysis of $6.6 \times 10^{19}$ POT ( $\sim 10 \%$ of the MiniBooNE neutrino data), has yielded a pure sample of neutral current elastic events. Of these, 5513 neutral current elastic events passed the selection criteria, were calibrated in kinetic energy, and analyzed to determine a value of the axial mass $M_{A}$ for NCE events in MiniBooNE. The value of $M_{A}=1.34_{-0.24}^{+0.38} \mathrm{GeV}$ for $\Delta s=0.0$ is in agreement with the result of the MiniBooNE CCQE analysis, as well as the BNL E734 result. As further analysis reduces errors, it will be interesting to observe whether the NCE result maintains agreement with the MiniBooNE CCQE result or the BNL E734 value. The CCQE $M_{A}$ is determined from the lepton vertex of the interaction, where this NCE result comes from the nucleon vertex. Similar results might indicate consistency of the nulcear model. Differences might indicate a non-zero value of $\Delta s$, final state interactions, or subtleties of 


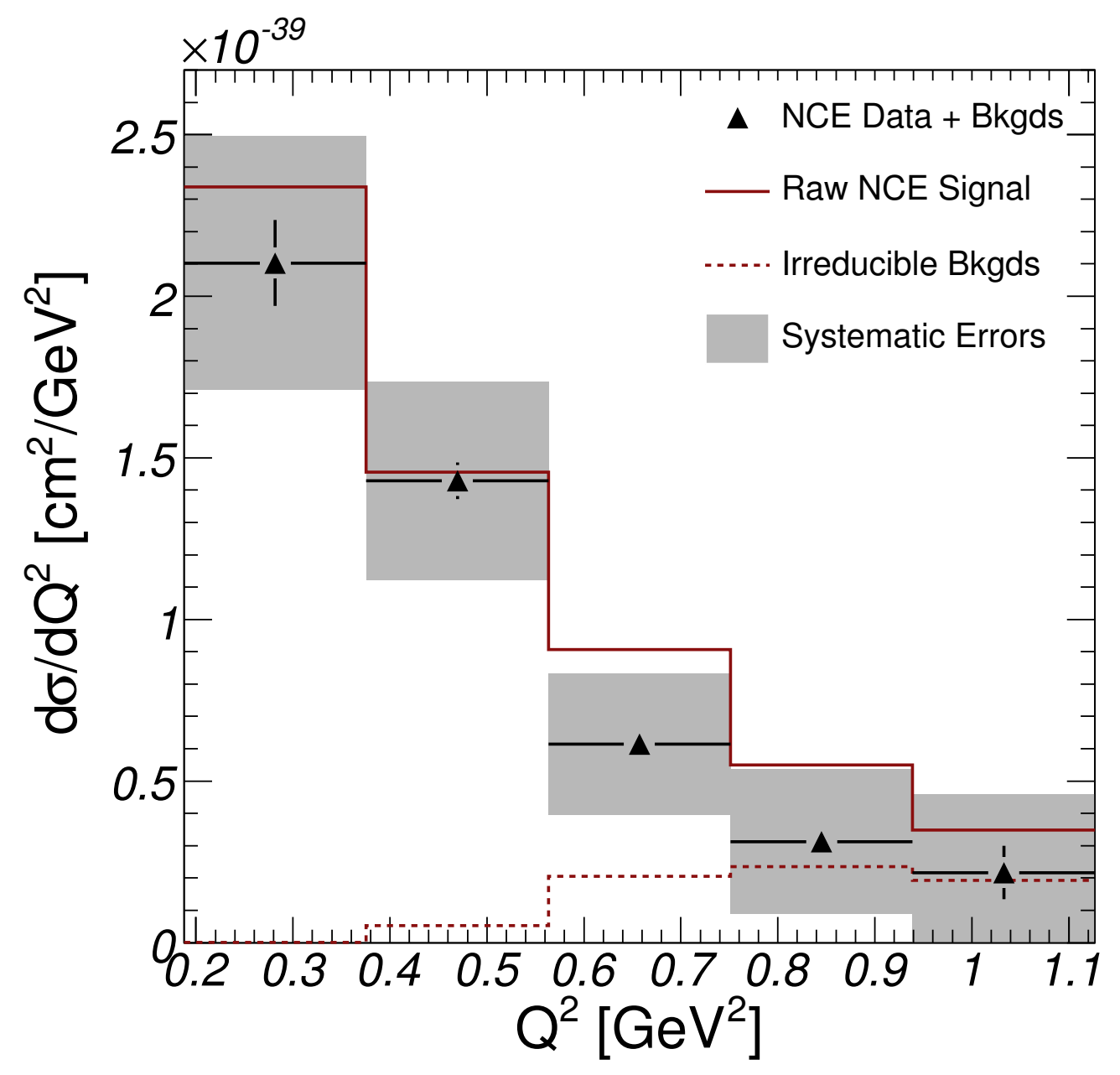

Figure 6.16: NCE data unfolded cross section with the NCE prediction from the Monte Carlo (solid histogram). The dotted histogram is the irreducible background prediction that was subtracted from the data before unfolding. 


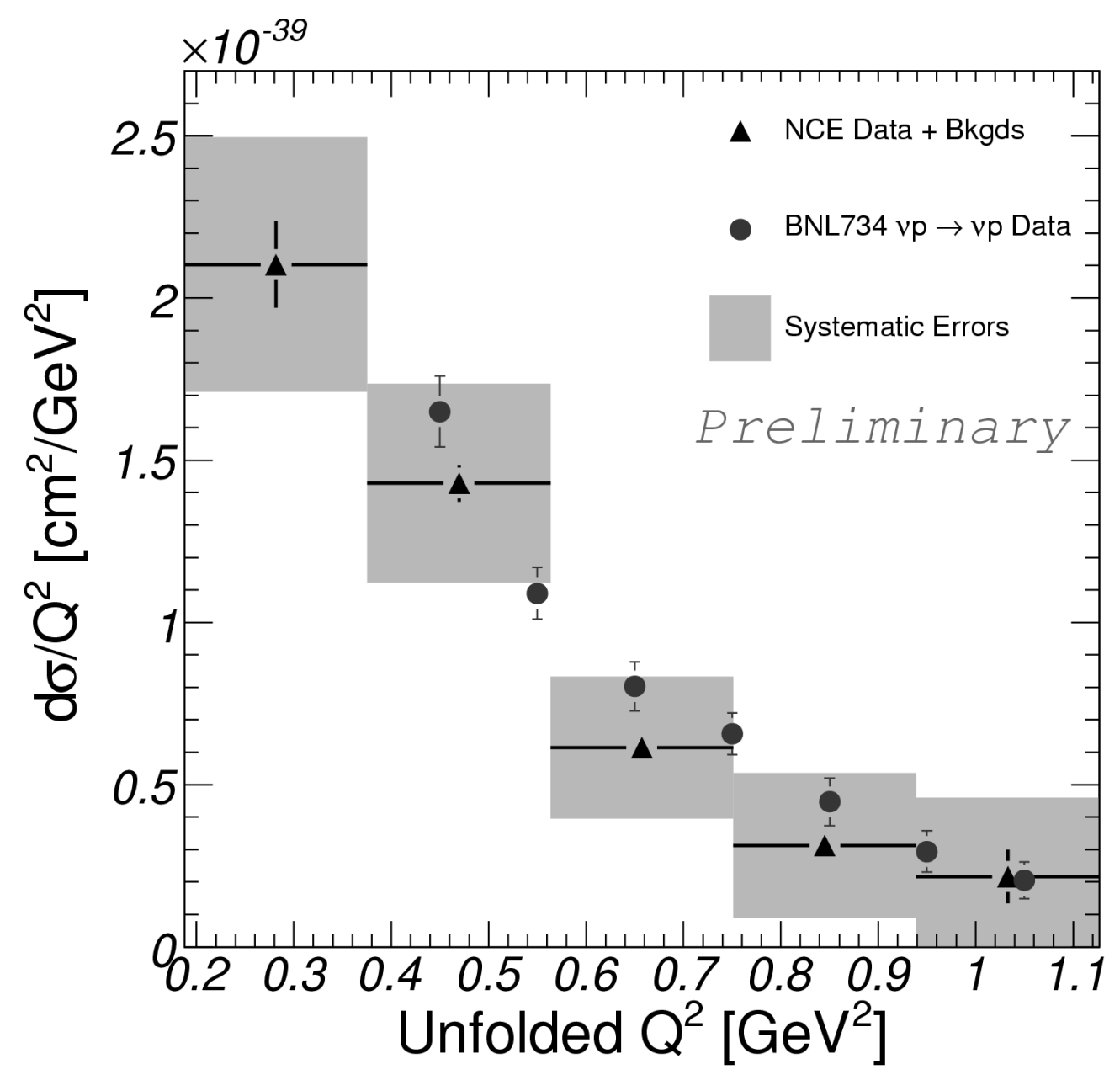

Figure 6.17: NCE data unfolded cross section with errors compared to the measurments from BNL 734 for $\nu$ p scattering. 
the neutral current interaction.

After subtracting backgrounds, 4137 NCE events were used to produce a differential cross section: the first such cross section with MiniBooNE data. It is important to reiterate that the irreducible backgrounds (events with the same final state as neutral current elastic events) were considered to be part of the background. The resultant differential cross section is reasonable and yields an integrated value of $\sigma\left(Q^{2}=.189 \rightarrow 1.13 \mathrm{GeV}^{2}\right)=$ $(8.8 \pm 0.6($ stat $) \pm 2.0($ syst $)) \times 10^{-40} \mathrm{~cm}^{2}$.

These results were produced from data with 4-5 times more events than any other neutrino-nucleon elastic scattering experiment, and they are only a subset of the total MiniBooNE data. Further analysis will include the full set of neutrino as well as antineutrino data, greatly increasing the statistical power of this result. Because blindness for oscillation results is not longer an issue, the range of reconstructed $Q^{2}$ is expected to increase as well. Improvements in the performance of reconstruction algorithms, background characterization and reduction, energy calibration, and unfolding procedures are expected to increase the quality of the NCE signal in these analyses.

The ability to measure these low energy protons well in a detector that was not designed for the task is encouraging in light of detectors that are better suited to it such as SciBooNE, Minerva, and FINeSSE. These neutrino experiments are well suited to the task of measuring the $s$ quark contribution to the nucleon in a complimentary way to electron scattering experiments. The comparison of the results from these very different types of experiments can only lead to better understanding of this physics. The MiniBooNE measurement of the neutral current elastic cross section and extraction of the nucleon axial mass is an important step in that direction. 


\section{Appendix A}

\section{Slow Monitoring}

The Slow Monitoring System (SMS) provides environmental monitoring of the detector,

vault, and support area, and warns the experiment in case of problems. This information is slow because it is recorded in seconds and minutes and averaged over minutes, days, and weeks. This appendix outlines MiniBooNE slow monitoring, though a complete treatment can be found in reference [134].

\section{A.1 Measured Quantities}

The Slow Monitoring System (SMS) was installed prior to MiniBooNE oil fill, and was operational before first beam to the detector. To ensure the quality and stability of measured data, critical quantities are monitored to ensure they remain within operational bounds. This information is displayed locally and in real time at the detector A.1 and is sampled for remote monitoring online A.3. This section describes the quantities measured. 


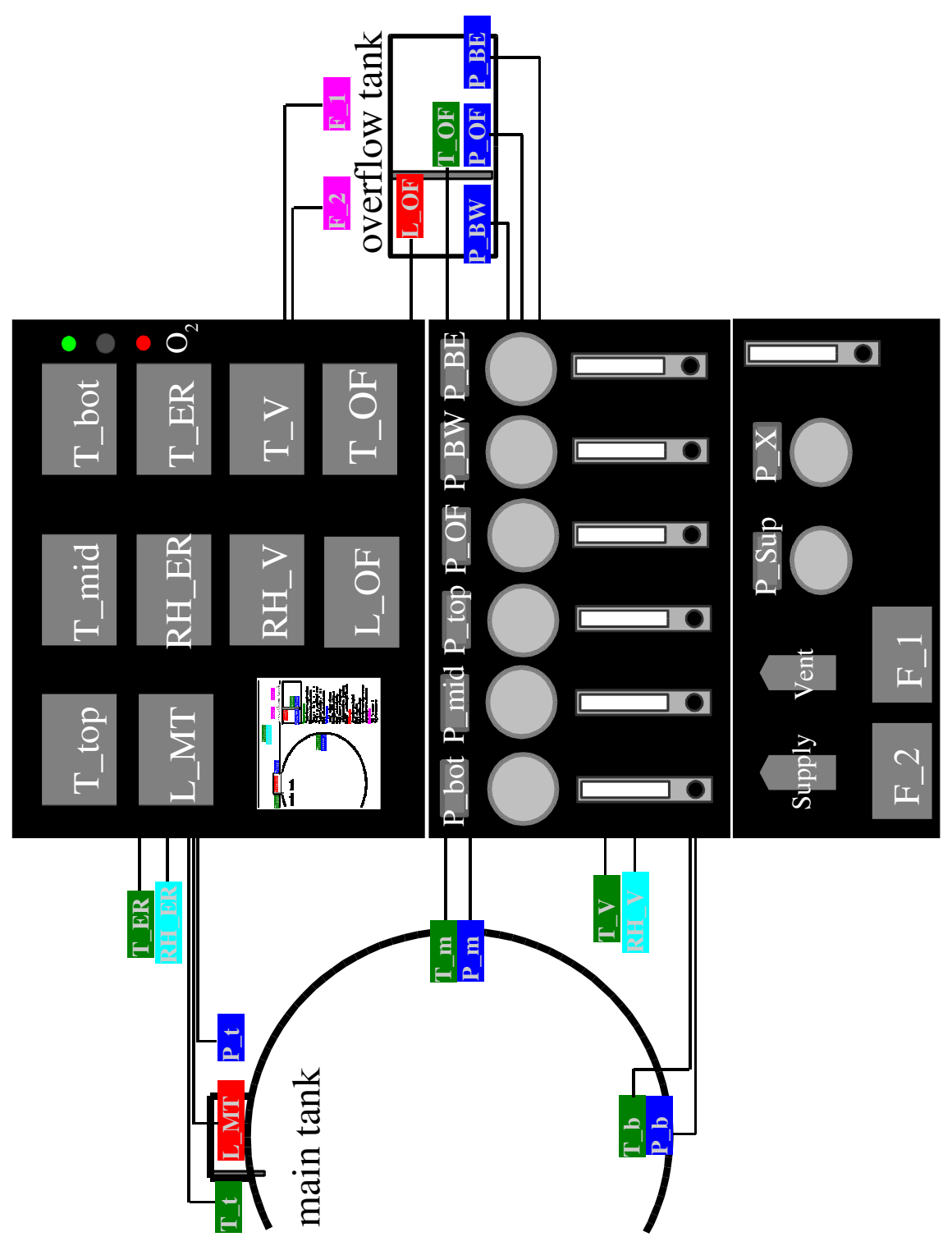

Figure A.1: Locations of slow monitoring probes in the detector and support building and the local display unit. Abbreviations are defined in table A.2. 


\section{A.1.1 Temperatures}

The SMS measures the temperatures of the oil, front-end electronics, and enclosure. Oil temperature is monitored at the bottom, equator, and access portal of the detector tank, as well as near the bottom of the buffer tank to within $\pm 1.1^{\circ} \mathrm{F}$. The temperature of the frontend electronics are measured in ${ }^{\circ} \mathrm{C}$ to the same accuracy $\pm 0.6^{\circ} \mathrm{C}$ and displayed above each rack. They are cooled directly by the MiniBooNE HVAC, and are monitored by a dedicated system that contacts the MiniBooNE control room and can shutdown the electronics in case of a problem. The HVAC provides secondary cooling for the enclosure (as well as the detector oil). Air temperatures in the vault and support area are monitored to $\pm 2.2^{\circ} \mathrm{F}$.

\section{A.1.2 Levels}

Oil level is also measured in each tank directly with capacitative level probes. Neither probe reads oil level in standard units, however the following conversions apply:

- a change of 1 unit by either probe corresponds to a change of $1.5 \pm 0.1 \in$ in level.

- The detector probe reads 15.6 when the oil is at the level of the overflow pipe in the access portal, and would read 4.5 when the oil level is at the very top of the detector sphere.

- Based on the geometry of the buffer tank, the level probe there is expected to read $\sim 45$ when full and $\sim 9$ when empty.

As the oil temperatures change so do volumes. The detector tank amplifies volume changes into height changes since it is shaped like an Erlenmeyer flask. Given $\Delta V / \Delta T=0.1 \% /{ }^{\circ} \mathrm{C}$, a change of $1^{\circ} \mathrm{F} \simeq 4.5 \in$ in the main tank. 


\section{A.1.3 Pressures}

Bubbling nitrogen gass through the oil was used to purge oxygen and other impurities. Currently it also provides a secondary measurement of oil level and an overpressure of nitrogen in the access portal. The pressures of the system supply and exhaust as well as the bubblers (when in use) are all monitored.

To provide a measurement of oil in the detector and buffer tank, nitrogen is bubbled through lines in each tank at slow rates such that the pressure of nitrogen in the line is equal to the gravitational pressure of the oil where the line terminates: $P_{N 2}=\rho_{\text {oil }} g h_{o i l}$ where $P_{N 2}$ is the measured pressure, $\rho_{o i l}$ is the oil density, and $h_{o i l}$ is the oil height above the line. This means, a change in pressure is equivalent to a change in height. In fact, given $\rho=0.855 \mathrm{~g} / \mathrm{cm}^{3}$ at $60{ }^{\circ} \mathrm{F}$ the conversion is $0.03 P S I=1$ in or $0.4 P S I=1 \mathrm{ft}$. The SMS measures 'tank pressures' at the top, middle, and bottom of the detector tank and at the bottom of the buffer tank. Oil levels calculated from the tank pressures are accurate to $\pm 2 \%$.

\section{A.1.4 Oil Flow Rate}

There are two flowmeters in the oil plumbing system. The first monitors the initial fill of oil to the buffer tank. The second tracks flow from the buffer to the detector tank. The oil flow readouts are in gallons per minute (GPM). However, the calibrations for the meters depend on the fluid viscosity, and therefore may not be exact. Even so, the flowmeters are accurate to an order of magnitude.

\section{A.1.5 Relative Humidities}

As well as measuring the air temperatures in the electronics room and vault, the SMS also measures relative humidity $(\mathrm{RH})$ in these enclosures. The humidity probes mount next to 
the temperature probes on the east wall of each room. Values are recorded in $\%$ relative humidity.

\section{A.1.6 Oxygen Levels}

Because nitrogen is constantly flowed into the detector, an oxygen monitor records the $\%$ oxygen in the support building as a safety measure. If the oxygen level drops below 19\%, the system shuts off nitrogen flow at the source.

\section{A.2 Nitrogen System}

MiniBooNE flows gaseous nitrogen into the detector oil system from an $\mathrm{LN}_{2}$ dewar outside the enclosure. Nitrogen is inert and so does not interact with the oil or the detector. Also it is both cheaper and safer than other inert gases because it composes roughly $80 \%$ of air. There are several purposes for nitrogen in MiniBooNE:

- Bubbled through the tanks, it purges moisture and more reactant gases like oxygen from the oil.

- It maintains a blanket of pure $\mathrm{N}_{2}$ over the oil in both tanks, displacing oxygen and other gases.

- It provides an indication of oil level in the tank

- It maintained a dry environment in the tanks until the oil fill was complete.

The system (shown in figure A.2 is based on a similar one used for LSND and reuses some of the components. It provides both monitoring and control of nitrogen flow to the detector and buffer tanks. The tanks are not allowed to vent individually. Instead, the exhaust lines from the main and buffer tanks are combined to a single line which has 


\begin{tabular}{|c|c|c|c|}
\hline Symbol & Quantity & Probe Location & Units and Error \\
\hline $\mathrm{T}_{M T t}$ & oil temp. & top portal MT & $\pm 1.1^{\circ} \mathrm{F}$ \\
\hline $\mathrm{T}_{M T m}$ & oil temp. & equator MT & $\pm 1.1{ }^{\circ} \mathrm{F}$ \\
\hline $\mathrm{T}_{M T b}$ & oil temp. & bottom MT & $\pm 1.1^{\circ} \mathrm{F}$ \\
\hline $\mathrm{T}_{O F}$ & oil temp. & bottom OF & $\pm 1.1^{\circ} \mathrm{F}$ \\
\hline $\mathrm{T}_{E R}$ & room temp. & ER, E wall & $\pm 2.2^{\circ} \mathrm{F}$ \\
\hline $\mathrm{T}_{V}$ & room temp. & Vault, E wall & $\pm 2.2^{\circ} \mathrm{F}$ \\
\hline $\mathrm{RH}_{E R}$ & room $\mathrm{RH}$ & ER, E wall & $\pm 2.5 \% \mathrm{RH}$ \\
\hline $\mathrm{RH}_{V}$ & room $\mathrm{RH}$ & Vault, E wall & $\pm 2.5 \% \mathrm{RH}$ \\
\hline $\mathrm{P}_{M T b}$ & $\mathrm{~N}_{2}$ press. & bottom MT, $1275 \pm 2 \mathrm{~cm} \mathrm{BWL}$ & $\mathrm{PSI}(0.4 \mathrm{PSI}=12.0 \pm 0.2 \mathrm{in})$ \\
\hline $\mathrm{P}_{M T m}$ & $\mathrm{~N}_{2}$ press. & equator MT, $656 \pm 1 \mathrm{~cm} \mathrm{BWL}$ & $\mathrm{PSI}(0.4 \mathrm{PSI}=12.0 \pm 0.2 \mathrm{in})$ \\
\hline $\mathrm{P}_{M T t}$ & $\mathrm{~N}_{2}$ press. & top portal MT, $57 \pm 1 \mathrm{~cm} \mathrm{BWL}$ & $\mathrm{PSI}(0.4 \mathrm{PSI}=12.0 \pm 0.2 \mathrm{in})$ \\
\hline $\mathrm{P}_{O F}$ & $\mathrm{~N}_{2}$ press. & bottom OF, $1 \mathrm{~cm} \mathrm{ABOF}$ & $\mathrm{PSI}(0.4 \mathrm{PSI}=12.0 \pm 0.2 \mathrm{in})$ \\
\hline $\mathrm{P}_{S}$ & $\mathrm{~N}_{2}$ press. & local display & PSI(not prop. to level) \\
\hline $\mathrm{P}_{X}$ & $\mathrm{~N}_{2}$ press. & local display & PSI(not prop. to level) \\
\hline $\mathrm{P}_{B W}$ & $\mathrm{~N}_{2}$ press. & bottom OF, West microbubbler & PSI(not prop. to level) \\
\hline $\mathrm{P}_{B E}$ & $\mathrm{~N}_{2}$ press. & bottom OF, East microbubbler & PSI(not prop. to level) \\
\hline $\mathrm{L}_{M T}$ & oil level & top hat MT & in. $(1 \mathrm{MTLMU}=1.5 \pm .1 \mathrm{in})$ \\
\hline $\mathrm{L}_{O F}$ & oil level & $\mathrm{OF}$ & in. $(1$ OFLMU $=1.5 \pm .1 \mathrm{in})$ \\
\hline $\mathrm{F}_{1}$ & oil flow rate & oil plumbing & GPM(calibration uncertain) \\
\hline $\mathrm{F}_{2}$ & oil flow rate & oil plumbing & GPM(calibration uncertain) \\
\hline $\mathrm{T}_{Q T}$ & air temp & QT, top & $\pm 0.6{ }^{\circ} \mathrm{C}$ \\
\hline
\end{tabular}

Table A.1: Summary of measured quantities. Abbreviations: MT = main detector tank, $\mathrm{OF}=$ overflow tank, $\mathrm{ER}=$ electronics room, $\mathrm{RH}=$ relative humidity, $\mathrm{BWL}=$ below weir level (level of overflow pipe in access portal), $\mathrm{ABOF}=$ above bottom of overflow tank 


\begin{tabular}{l|l}
\hline Code & Full Name \\
\hline $\mathrm{SMS}$ & Slow Monitoring System \\
$\mathrm{T}_{M T t}$ & Temperature: Main Tank: top \\
$\mathrm{T}_{M T m}$ & Temperature: Main Tank: middle \\
$\mathrm{T}_{M T b}$ & Temperature: Main Tank: bottom \\
$\mathrm{T}_{O F T}\left(\mathrm{~T}_{O F}\right)$ & Temperature: OverFlow Tank \\
$\mathrm{T}_{E R}$ & Temperature: Electronics Room \\
$\mathrm{RH}_{E R}$ & Relative Humidity: Electronics Room \\
$\mathrm{T}_{V}$ & Temperature: Vault \\
$\mathrm{RH}_{V}$ & Relative Humidity: Vault \\
$\mathrm{L}_{O F}$ & Level: OverFlow Tank \\
$\mathrm{L}_{M T}$ & Level: Main Tank \\
$\mathrm{P}_{O F T}\left(\mathrm{P}_{O F}\right)$ & Pressure: OverFlow Tank line \\
$\mathrm{P}_{M T t}$ & Pressure: Main Tank: top line \\
$\mathrm{P}_{M T m}$ & Pressure: Main Tank: middle line \\
$\mathrm{P}_{M T b}$ & Pressure: Main Tank: bottom line \\
$\mathrm{P}_{B W}$ & Pressure: Bubbler West \\
$\mathrm{P}_{B E}$ & $\mathrm{P}_{S}$ \\
$\mathrm{P}_{X}$ & Plows : pump1 \\
$\mathrm{F}_{1}$ & Prowe: Supply \\
\hline
\end{tabular}

Table A.2: Slow Monitoring abbreviations. 


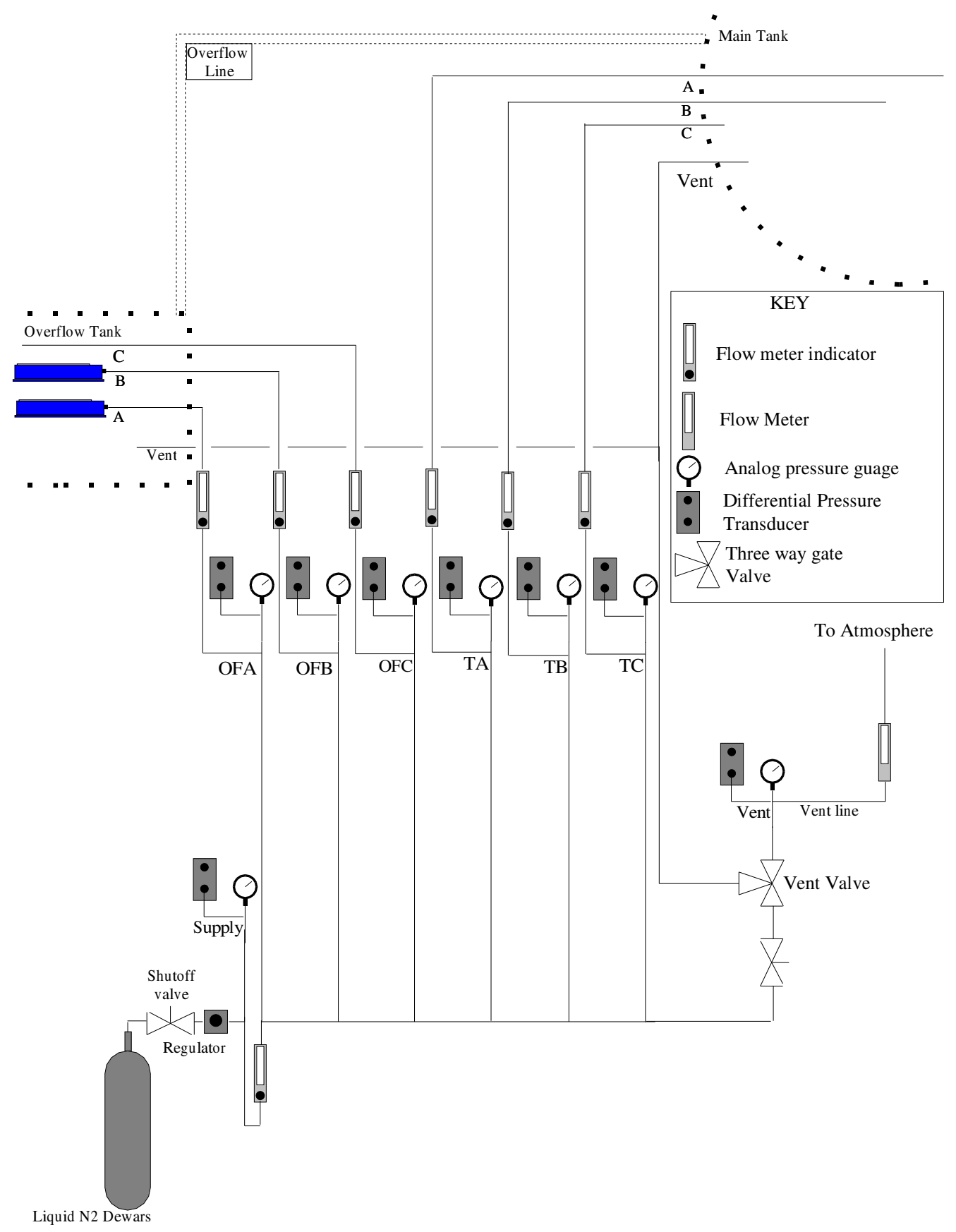

Figure A.2: The N2 system is contained within the slow monitoring rack and will give us secondary indication of tank level and aid in removing water and $\mathrm{O} 2$ from the system. 
both an overpressure and an underpressure valve. The flow and pressure of the exhaust is monitored before it vents to the outside. The pressure is consistent with atmospheric.

\section{A.2.1 Nitrogen Bubbling}

There are two micro-bubblers in the buffer tank. Valves on the local slow monitoring display rack determine whether nitrogen is directed towards these. To ensure nitrogen saturation of all the detector oil, the bubblers run while the oil is circulated between the main and buffer tanks.

During the summer 2002, nitrogen was bubbled at a high rate through the tanks to purge oxygen from the oil. The physics of purging is simple. Small bubbles of pure $\mathrm{N}_{2}$ flow through the oil. The partial pressure of $\mathrm{N}_{2}$ in the bubbles is high, while the partial pressure of $\mathrm{O}_{2}$, and other gases is low. Oxygen and other gases diffuse into the bubbles until they reach equilibrium. If enough nitrogen flows through the oil, only a minimal amount of oxygen should remain.

H.-O. Meyer calculated that a successful purge required .9 million liters of nitrogen gas, or 30 LPM for 20 days [135]. MiniBooNE bubbled 30 LPM for 26 days, or 1.1 million liters.

\section{A.2.2 Nitrogen Blanket}

Having purged oxygen and other harmful gases from the oil, it is necessary to maintain a blanket of nitrogen above the oil. Keeping such a blanket prevents other gases from diffusing into the oil and maintains the partial pressure of nitrogen achieved by bubbling. This is accomplished using the nitrogen that flows slowly into the detector as a secondary measurement of oil level. 


\section{A.2.3 Level Monitoring}

As mentioned in the previous section, oil level in the detector and buffer tanks can be measured by flowing small amounts of nitrogen through small lines that terminate in the tanks. The detector tank level is measured in three places via this method, the overflow tank in one place. The pressure required to push the nitrogen through the lines equals the gravitational pressure of the oil pushing back: $P_{N 2}=\rho_{o i l} g h_{o i l}$. So given $\rho_{\text {oil }}=0.854 \mathrm{~g} / \mathrm{cm}^{3}$ at $60^{\circ} \mathrm{F}$, then $0.4 \mathrm{PSI}=12.0 \pm 0.2 \mathrm{in}$.

\section{A.2.4 Pre Oil Fill}

Prior to oil fill, two dewars of liquid nitrogen were pumped into the overflow tank to remove excess moisture. Water vapor became trapped in the tank when it was sealed after its initial steam cleaning. Each dewar flowed nitrogen into the tank through the open line $\mathrm{N}_{2} \mathrm{OF}$ at 5 SCFH which was simultaneously vented through another line to prevent pressurizing the tank.

\section{A.3 Remote Monitoring System}

In order to monitor the detector slow information remotely from the MiniBooNE control room (and elsewhere), an off-the-shelf system (called webDAQ/100) was installed to sample and record these data. All of the variables described so far with the exception of the front-end electronics temperatures and the oxygen level are routed to this device. The electronics racks are monitored by another system that also oversees the HVAC. It retains 24 hours of information of temperature information and can shutdown the electronics if the temperatures exceed operating limits.

The webDAQ takes as input voltages output from each of the gauges on the local display rack. These are simply converted back to their physical units with a linear scaling 
(table A.3). The values are sampled every second and averaged for ten minutes before being written to its memory.

Although the webDAQ was configured from its webpage interface, this was found to be an unsatisfactory interface for monitoring data trends. Instead, several programs running on a dedicated computer read the webDAQ data, save it to a database, and make plots of data trends. These plots (one for the past $24 \mathrm{hrs,} \mathrm{one} \mathrm{for} \mathrm{the} \mathrm{past} 7$ days, and one for the past month) are updated in 30 minute intervals and made available online for monitoring by MiniBooNE personel. Figure A.3 gives an example of a such a plot.

\section{A.4 Summary}

The MiniBooNE Slow Monitoring System works and is currently monitoring the detector. It measures electronics and oil temperatures, oil levels and flow, and air temperatures and humidities. It also controls and measures nitrogen flow through the tank. All of these values are displayed locally exported via the webDAQ to the slow monitoring web page. 


\begin{tabular}{l|l|ll|l} 
chan\# & name & slope & intercept & physical unit \\
\hline $\mathrm{C} 0$ & wDAQtime & 1.0 & 0.0 & seconds \\
$\mathrm{C} 1$ & $\mathrm{~T}_{M T t}$ & 100.72 & 9.6653 & degF \\
$\mathrm{C} 2$ & $\mathrm{~T}_{M T m}$ & 100.53 & 10.035 & $\operatorname{degF}$ \\
$\mathrm{C} 3$ & $\mathrm{~T}_{M T b}$ & 100.12 & 10.508 & $\operatorname{degF}$ \\
$\mathrm{C} 4$ & $\mathrm{~T}_{O F T}$ & 101.71 & 10.153 & $\operatorname{degF}$ \\
$\mathrm{C} 5$ & $\mathrm{~T}_{E} R$ & 99.038 & 12.357 & degF \\
$\mathrm{C} 6$ & $\mathrm{RH}_{E} R$ & 10.706 & 2.2195 & \%humidity \\
$\mathrm{C} 7$ & $\mathrm{~T}_{V}$ & 99.586 & 13.491 & degF \\
$\mathrm{C} 8$ & $\mathrm{RH}_{V}$ & 10.598 & 1.9886 & \%humidity \\
$\mathrm{C} 9$ & $\mathrm{~L}_{O} F$ & 9.2462 & -15.208 & OFLMU \\
$\mathrm{C} 10$ & $\mathrm{~L}_{M} T$ & 5.3344 & -9.7914 & MTLMU \\
$\mathrm{C} 11$ & $\mathrm{P}_{O} F T$ & 1 & -1 & PSI \\
$\mathrm{C} 12$ & $\mathrm{P}_{M} T t$ & 1 & -1 & PSI \\
$\mathrm{C} 13$ & $\mathrm{P}_{M} T m$ & 3 & -3 & PSI \\
$\mathrm{C} 14$ & $\mathrm{P}_{M} T b$ & 6 & -6 & PSI \\
$\mathrm{C} 15$ & $\mathrm{P}_{B} W$ & 6 & -6 & PSI \\
$\mathrm{C} 16$ & $\mathrm{P}_{B} E$ & 6 & -6 & PSI \\
$\mathrm{C} 17$ & $\mathrm{P}_{S}$ & 6 & -6 & PSI \\
$\mathrm{C} 18$ & $\mathrm{P}_{X}$ & 0.722 & -0.722 & PSI \\
$\mathrm{C} 19$ & $\mathrm{~F}_{2}$ & $225 / 47$ & -9 & gal/min \\
\hline
\end{tabular}

Table A.3: Voltage to physical unit conversion factors for webDAQ data. 


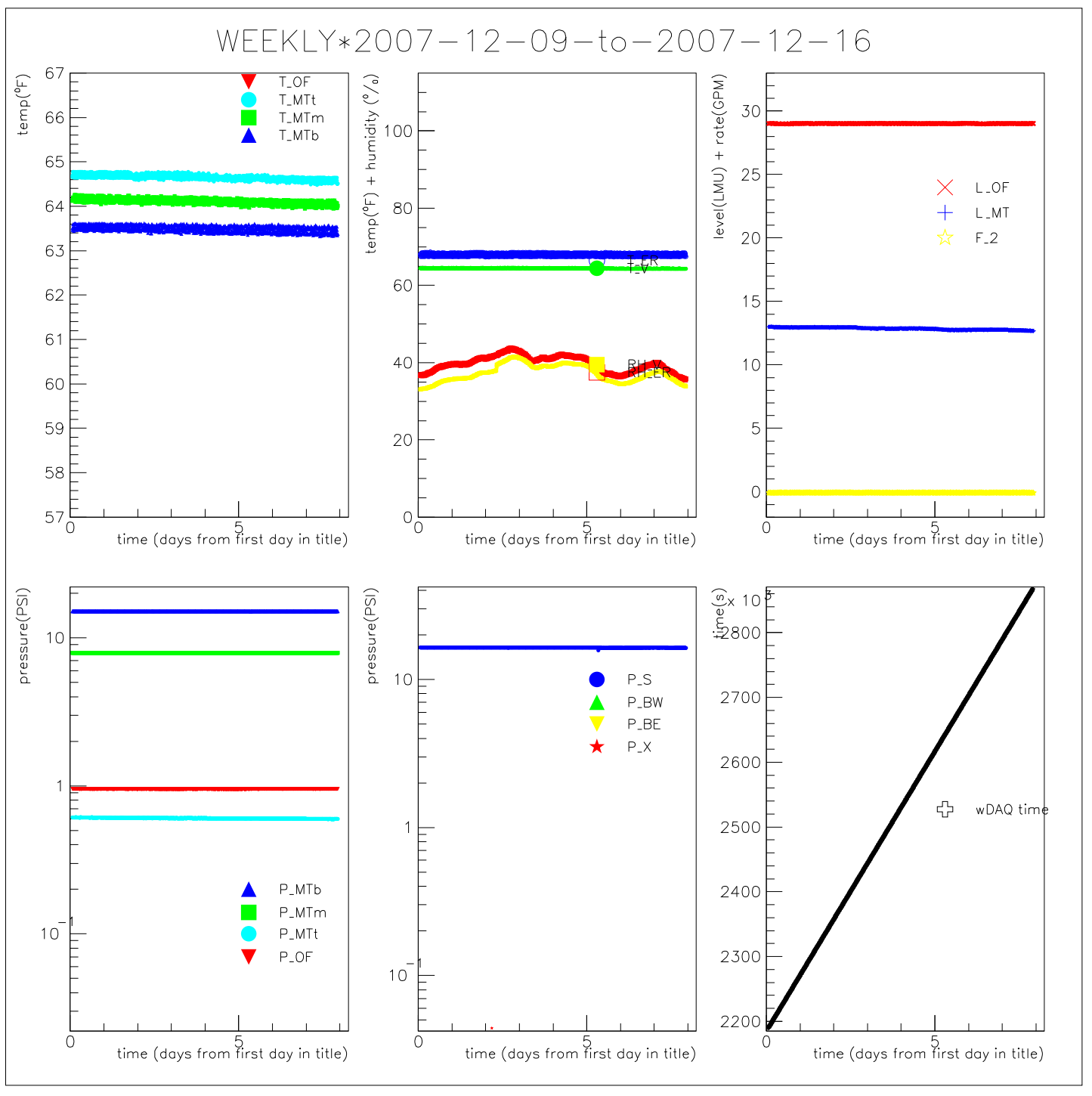

Figure A.3: A week's worth of slow monitoring data. Note the stability of most quantities. 


\section{Bibliography}

[1] W. Pauli, Letter to the Physical Society of Tubingen (1930), Reproduced in Brown, L. M. Physics Today, 31,23 (1978).

[2] F. Reines and C. L. Cowan, Phys. Rev. 92:830 (1953).

[3] C. L. Cowan et al., Science 124:103 (1956).

[4] G. Danby et al., Phys. Rev. Lett. 9:36 (1962).

[5] [DONUT collaboration], K. Kodama et al., Phys. Lett. B 504:218 (2001).

[6] B. Pontecorvo, Sov. Phys.--JETP 33:549 (1957).

[7] B. Pontecorvo, Sov. Phys.--JETP 34:247 (1958).

[8] Y. Fukuda et al., Phys. Rev. Lett. 81:1562 (1998).

[9] AAAS, The Standard Model, http://www.hep.phy.cam.ac.uk/ drw1/ particleandnuclear/welcome.html.

[10] D. Binosi and L. Thelussl, Comp. Phys. Comm. 161:76 (2004).

[11] R. N. Mohapatra and G. Senjanović, Phys. Rev. Lett. 44:912 (1980).

[12] S. F. King, JHEP 9 (2002).

[13] R. Davis, Prog. Part. Nucl. Phys. 32:13 (1994). 
[14] [GALLEX collaboration], W. Hampel et al., Phys. Lett. B 447:127 (1999).

[15] [SAGE collaboration], J. N. Abdurashitov et al., Sov. Phys.-JETP 95:181 (2002).

[16] S. Fukuda et al., Phys. Rev. Lett. 86:5656 (2001).

[17] [KamLAND collaboration], T. Araki, Phys. Rev. Lett. 94:081801 (2005).

[18] [SNO collaboration], B. Aharmim et al., Phys. Rev. D 72:052010 (2005).

[19] K. S. Hirata et al., Phys. Lett. B 280:146 (1992).

[20] [Super-Kamiokande collaboration], Y. Ashie et al., Phys. Rev. D 71:112005 (2005).

[21] [K2K collaboration], M. Ahn et al., Phys. Rev. D 74:072003 (2006).

[22] [MINOS collaboration], D. Michael et al., Phys. Rev. Lett. 97:191801 (2006).

[23] [LSND collaboration], A. Aguilar et al., Phys. Rev. D 64:112007 (2006).

[24] D. Karlen, Euro. Phys. Jour. C 15:357 (2000).

[25] [MiniBooNE collaboration], A. Aguilar-Arevalo et al., Phys. Rev. Lett. 98:231801 (2007).

[26] E. Laird, R. Patterson, and H. Tanaka, A Proposal for Unblinding Two Sideband Regions in the Likelihood-Based Oscillation Analysis, BooNE Technical Note 216 (2006).

[27] B. Roe et al., Nucl. Inst. \& Meth. A 543:577 (2005).

[28] S. Weinberg, Phys. Rev. Lett. 19:1264 (1967).

[29] A. Salam and J. C. Ward, Phys. Lett. 13:168 (1964).

[30] S. Glashow, Rev. Mod. Phys. 52:539 (1980).

[31] F. J. . Hasert et al., Phys. Lett. B 46:121 (1973). 
[32] F. J. . Hasert et al., Phys. Lett. B 46:138 (1973).

[33] W. Lee et al., Phys. Rev. Lett. 37:186 (1976).

[34] D. Cline et al., Phys. Rev. Lett. 37:252 (1976).

[35] D. Cline et al., Phys. Rev. Lett. 37:648 (1976).

[36] A. Entenberg et al., Phys. Rev. Lett. 42:1198 (1979).

[37] H. Faissner et al., Phys. Rev. D 21:555 (1980).

[38] P. Coteus et al., Phys. Rev. D 24:1420 (1981).

[39] J. Horstkotte et al., Phys. Rev. D 25:2743 (1982).

[40] L. A. Ahrens et al., Phys. Rev. D 35:785 (1987).

[41] [SNO collaboration], Q. Ahmad et al., Phys. Rev. Lett. 89:011301 (2002).

[42] C. Horowitz, Phys. Rev. D 65:043001 (2002).

[43] C. Horowitz, Electroweak Scattering in Heaven and Earth (2002).

[44] K. Langanke, P. Vogel, and E. Kolbe, Phys. Rev. Lett. 76:2629 (1996).

[45] J. Beacom, W. Farr, and P. Vogel, Phys. Rev. D 66:033001 (2002).

[46] R. Jaffe and C. Korpa, Comments Nucl. Part. Phys. 17:163 (1987).

[47] [NuTeV collaboration], T. Adams, arXiv:hep-ex/9906038v1 (1999).

[48] [HERMES collaboration], A. Airapetian, Phys. Rev. D 75:012007 (2007).

[49] T. Katori, Llewellyn Smith's Formalism, BooNE Memo: http://wwwboone.fnal.gov/memos/TK_llewllyn.pdf (2005).

[50] [BLAST collaboration], R. Alarcon et al., AIP Conf. Proc. 842:315 (2006). 
[51] E. Geis, The Electric Form Factor of the Neutron at Low Momentum Transfers as Measured at Bates Large Acceptance Spectrometer Toroid, Ph.D. thesis, Arizona State University (2007).

[52] [CLAS collaboration], W. Brooks and J. Lachniet, Phys. Lett. A 755:261 (2005).

[53] [SAMPLE collaboration], D. Spayde et al., Phys. Lett. B 583:79 (2004).

[54] F. Maas et al., Phys. Rev. Lett. 94:152001 (2005).

[55] [G0 collaboration], D. Armstrong et al., Phys. Rev. Lett. 95:092001 (2005).

[56] [HAPPEX collaboration], A. Acha et al., Phys. Rev. Lett. 98:032301 (2007).

[57] B. Filippone and X. Ji, arXiv:hep-ph/0101224 (2001).

[58] [SMC collaboration], D. Adams et al., Phys. Rev. D 56:5330 (1997).

[59] F. Halzen and A. Martin, Quarks \& Leptons, John Wiley \& Sons (1984).

[60] C. H. Llewellyn-Smith, Phys. Rep. 3C:261 (1972).

[61] G. Garvey et al., Phys. Rev. C 48:1919 (1993).

[62] W. Alberico, Acta Phys. Polon B37:2269 (2006).

[63] N. Jachowicz et al., Phys. Rev. C 76:055501 (2007).

[64] G. Garvey, W. Louis, and D. White, Phys. Rev. C 48:761 (1993).

[65] W. Alberico et al., Nucl. Phys. A 651:277 (1999).

[66] S. Pate, Phys. Rev. Lett. 92:082002 (2004).

[67] L. Bugel et al., A Letter of Intent for a Neutrino Scattering Experiment on the Booster Neutrino Beamline: FINeSSE, http://www-finesse.fnal.gov/ (2005). 
[68] Minerva, http://minerva.fnal.gov/.

[69] J. Conrad et al., Bringing the SciBar Detector to the Booster Neutrino Beam, http: //www-sciboone.fnal.gov/ (2006).

[70] I. Stancu et al., Technical Design Report for the 8 GeV Beam, http://www-boone. fnal.gov/publicpages/ (2001).

[71] J. Monroe et al., Measuring Protons on Target at MiniBooNE, BooNE Technical Note 87 (2003).

[72] I. Stancu et al., Technical Design Report for the MiniBooNE Neutrino Beam, http: //www-boone.fnal.gov/beam/ (2001).

[73] T. Kobilarcik, J. DeVoy, and C. Moore, Automatic Beamline Correction, proceedings of the 2003 Particle Accelerator Conference (2003).

[74] L. Bugel and M. Sorel, Magnetic Field Measurments for the MiniBooNE Prototype Horn, BooNE Technical Note 34 (2001).

[75] [GEANT4 collaboration], S. Agostinelli et al., Nucl. Inst. \& Meth. A 506:250, http: //wwwasd.web.cern.ch/wwwasd/geant4/G4UsersDocuments/Overview/html/ (2003).

[76] L. Bartoszek, http://www.bartoszekeng.com, Bartoszek Engineering.

[77] S. Brice et al., Predicted and Observed Neutrino Rates in Horn-On and Horn-Off Running Modes, BooNE Technical Note 119 (2004).

[78] E. D. Zimmerman et al., LMC Technical Design Report.

[79] I. Stancu et al., The MiniBooNE Detector Technical Design Report, http:// WwW-boone.fnal.gov/publicpages/ (2001). 
[80] Hamamatsu, http://hamamatsu.com (2001).

[81] B. Fleming and D. Smith, MiniBooNE PhotoMultiplier Tube Testing, BooNE Technical Note 30 (2002).

[82] S. Breitzmann and H. O. Meyer, Solubility of O2, N2, and Ar in Mineral Oil, BooNE Technical Note 65 (2002).

[83] R. Tayloe and J. A. Green, MiniBooNE Trigger Notes v. 5.0, http://www-boone. fnal.gov/operation/DAQ_trigger/trigger/trigger_notes_V5.0.1.html (2005).

[84] W. R. Leo, Techniques for Nuclear and Particle Physics Experiments, Springer-Verlag (1987).

[85] Hamamatsu, http://sales.hamamatsu.com/en/products/ electron-tube-division/detectors/photomultiplier-tubes/part-r5912.php (2001).

[86] R. Patterson, The PMT Charge Calculation, BooNE Technical Note 83 (2003).

[87] M. O. Wascko, Energy Calibration of MiniBooNE with Cosmic Muons, http:// home.fnal.gov/ ${ }^{\sim}$ wascko/boone/misc/tollestrup.pdf (2004).

[88] R. Imlay et al., Checking the Directional Reconstruction Performance of the Fitters Using the Muon Tracker, BooNE Technical Note 99 (2003).

[89] R. Imlay et al., Measuring the Energy of Muons in MiniBooNE with the Cubes, BooNE Technical Note 106 (2003).

[90] H. O. Meyer, Index of Refraction of Marcol 7, BooNE Technical Note 90 (2003).

[91] J. B. Birks, The Theory and Practice of Scintillation Counting (1964). 
[92] P. Ockerse et al., Tests of the Scintillation Properties ofMineral Oil at IUCF, BooNE Technical Note 74 (2003).

[93] J. Raaf, A Measurement of the Neutrino Neutral Current $\pi^{0}$ Cross Section at MiniBooNE, Ph.D. thesis, University of Cincinnati (2005).

[94] M. Sorel, Search for Sterile Neutrinos Using the MiniBooNE Beam, Ph.D. thesis, Columbia University (2005).

[95] D. Toptygin, Time-Resolved Fluorescence of MiniBooNE Mineral Oil, BooNE Technical Note 122 (2004).

[96] B. C. Brown et al., Study of Scintillation, Fluorescence and Scattering in Mineral Oil for the MiniBooNE Neutrino Detector, IEEE Nuclear Science Symposium Conference Record (2003).

[97] E. Hecht, Optics, Addison-Wesley (1998).

[98] B. Brown et al., Studies of the Optical Properties of Marcol 7 Mineral Oil, BooNE Technical Note 145 (2004).

[99] N. V. Mokhov and S. I. Striganov, MARS15 Overview, Hadronic Shower Simulations Workshop (2006).

[100] J. R. Sanford and C. L. Wang, BNL AGS Internal Report p. 11299 (1967).

[101] I. Chemakin et al., Nucl. Phys. A 639:407 (1998).

[102] [HARP collaboration], M. Catanesi, Eur. Phys. J. C 52:29, arXiv:hep-ex/0702024 (2007).

[103] M. Tzanov, Determination of the $\pi^{+}$Production Cross Section from External Data, BooNE Technical Note 204 (2006). 
[104] D. Schmitz, A measurement of hadron production cross-sections for the simulation of accelerator neutrino beams and a search for $\nu_{\mu} \rightarrow \nu_{e}$ oscillations in the $1 \mathrm{~m}^{2} \mathrm{eV}^{2}$ region, Ph.D. thesis, Columbia University (2008).

[105] M. H. Shaevitz, Determination of The $K^{+}$Production Cross Section at 8 GeV Using External Data, BooNE Technical Note 209 (2006).

[106] A. Aleshin et al., ITEP-77-80 (1977).

[107] J. V. Allaby et al., CERN 70-12, Nuclear Physics Division (1970).

[108] D. Dekkers et al., Phys. Rev. 137:962 (1965).

[109] R. A. Lundy et al., Phys. Rev. Lett. 14:504 (1965).

[110] G. J. Marmer et al., Phys. Rev. 179 (1969).

[111] I. A. Vorontsov et al., ITEP-88-11 (1988).

[112] [E-802 collaboration], T. Abbott et al., Phys. Rev. D 45:3906 (1992).

[113] P. A. Piroue et al., Phys. Rev. 148 (1966).

[114] T. Eichten et al., Nucl. Phys. B 44 (1972).

[115] D. Casper, Nucl. Phys. B (Proc. Suppl.) 112:161, http://nuint.ps.uci.edu/ nuance/ (2002).

[116] R. A. Smith and E. J. Moniz, Nucl. Phys. B 43:605, Erratum-ibid. B101:547 (1975) (1972).

[117] D. Rein and L. M. Sehgal, Nucl. Phys. B 223:29 (1983).

[118] D. C. Cox et al., A Guide to Neutrino Cross Sections at MiniBooNE (2003).

[119] A. Aguilar-Arevalo et al., Phys. Rev. Lett. 100:032301 (2008). 
[120] T. Katori, R. Tayloe, and G. Zeller, Update on the MiniBooNE $\nu_{\mu}$ QE Analysis, BooNE Technical Note 219 (2006).

[121] R. Patterson, A Search for Muon Neutrino to Electron Neutrino Oscillations at $\Delta m^{2}>1 e V^{2}$, Ph.D. thesis, Princeton University (2007).

[122] [GCALOR collaboration], C. Zeitnitz and T. A. Gabriel, Nucl. Inst. \& Meth. A 349:106, http://www.npl.uiuc.edu/exp/G0/computation/gcalor.html (1994).

[123] H. Tanaka, Systematic Uncertainties due to the Spread and Divergence of the Primary Beam and p-Be Hadronic Interactions, BooNE Technical Note 177 (2006).

[124] J. Monroe, A Combined $\nu_{\mu}$ and $\nu_{e}$ Oscillation Search at MiniBooNE, Ph.D. thesis, Columbia University (2006).

[125] Neutrino Cross Section Uncertainties: What We Know About What We Don't Know, BooNE Technical Note 102 (2003).

[126] P. Kasper, SplitEvent Performance, BooNE Memo: http://wwwboone.fnal.gov/memos/SEperf.html A complete description of the SplitEvent algorithm can be found in the Analysis Framework Users Manual (2005).

[127] I. Stancu, Performance of the Reconstruction Algorithms on the Neutral-Current Data, BooNE Technical Note 108 (2003).

[128] I. Stancu, StancuFitters, http://www-boone.fnal.gov/software_and_analysis/ current_framework/doc/UserManual/StancuFitters.html.

[129] I. Stancu, Maximum Likelihood Event Reconstruction Algorithms in MiniBooNE, BooNE Technical Notes 18,19,21,50 (1999-2002).

[130] F. James and M. Roos, Comp. Phys. Comm. 10:343 (1975). 
[131] K. Mahn et al., EventWeight and MultisimMatrix: a guide to the reweighting and MiniBooNE error analysis with multisims, BooNE Technical Note 231 (2007).

[132] A. Aquilar-Arevalo et al., MiniBooNE Final Oscillation Fits to nue and numu Events, BooNE Technical Note 212 (2006).

[133] G. Cowan, Statistical Data Analysis (1998).

[134] C. Cox et al., Slow Monitoring and Nitrogen Systems for MiniBooNE, Version II, BooNE Technical Note 70 (2002).

[135] H. Meyer, Remarks on Nitrogen Bubbling, BooNE Memo (2002). 
MNB Technologies, Inc. 1194 E Winners Cir.

D. Christopher Bloomington, IN 47401 Cox

Work: (812) 824-8226

Cell: (812) 345-9388

Fax: (812) 824-8230

ccox@mnbtech.com

EDUCATION

Indiana University, Bloomington, IN 2001-2008

Ph.D. Physics

Dissertation: "A Measurement of The Neutral Current Neutrino-

Nucleon Elastic Cross Section at MiniBooNE"

Indiana University, Bloomington, IN $\quad 2000-2001$

M.S. Physics

Towson University, Towson, MD

$1996-2000$

B.S. Physics, summa cum laude

ACADEMIC EXPERIENCE

Research Assistant, 2001 - 2008, Indiana Univeristy

Associate Instructor, 2000 - 2001, Indiana University

HONORS AND AWARDS

Outstanding Graduate Student in Experimental 2006

Research Award (IU Physics)

Indiana University College of Arts and Sciences 2003

Travel Grant 
George Michail Memorial Poster Award 2002

William H. Pelham Award for Outstanding 1999

Juniors in Physics, Astronomy, and Geosciences

College of Natural and Mathematical $\quad 1996-2000$

Sciences Dean's Scholarship

Towson University Scholarship $\quad 1996-2000$

Professional Activites

President $2002-2003$

Indiana University Physics Graduate Student Association

Student Representative $2003-2006$

Physics Department Colloquium Committee

Student Representative $\quad 2002-2003$

Physics Department Curriculum Committee

Student Representative $2002-2003$

IU Cyclotron Student, Staff, Faculty Relations Committee

Student Representative $2001-2002$

Physics Department Student, Staff Faculty Relations Committee

$\begin{array}{ll}\text { President } & 1999-2000\end{array}$

Towson University Chapter Society of Physics Students

Secretary/Treasurer $\quad 1998-1999$

Towson University Chapter Society of Physics Students 
Indiana Univeristy Physics and Astronomy Open House

Professional Affiliations

American Physics Society (APS)

Society of Physics Students (SPS)

Sigma Pi Sigma $(\Sigma \Pi \Sigma)$

Golden Key National Honor Society

TeChnical Skills

\section{Operating Systems}

Linux, Unix, Windows

Programming Languages

C++, C, FORTRAN, Perl, C-shell, AWK, HTML, Visual Basic, SQL

Software

ROOT, PAW, CERNLIB, LATEX, Microsoft Office, Open Office, LabVIEW, Emacs, Vi

Hardware Experience

Machine Shop Tools, Computer Assembly

\section{Presentations}

NSF Review of the IUCF Nuclear Physics Group, Bloomington, IN, Poster: "NeutrinoNucleon Elastic Scattering in MiniBooNE", 11/29/2007 
Division of Nuclear Physics Meeting, Newport News, VA, "A first measurement of neutrino nucleon elastic scattering in MiniBooNE", 10/13/2007

Fifth International Workshop on Neutrino-Nucleus Interactions in the Few-GeV Region, Fermilab, Batavia, IL, "Neutrino Nucleon Elastic Scattering in MiniBooNE", $5 / 31 / 2007$

Division of Nuclear Physics Meeting, Nashville, TN, "Neutrino Nucleon Elastic Scattering in MiniBooNE", 10/25/2006

18th National Nuclear Physics Summer School, Bloomington, IN, Student Presentation: "Neutrino Nucleon Elastic Scattering Cross Section in MiniBooNE", $8 / 1 / 2006$

NSF Review of the IUCF Nuclear Physics Group, Bloomington, IN, Poster: "Neutrino Nucleon Elastic Scattering in MiniBooNE", 11/16/2004

Division of Nuclear Physics Meeting, Chicago, IL, "Neutrino Nucleon Elastic Scattering in MiniBooNE", 10/29/2004

Sixteenth Summer School in Nuclear Physics, Bar Harbor, ME, Student Presentation: "Neutrino Nucleon Elastic Scattering and the Strange Quark Component of Nucleon Spin in MiniBooNE", 6/16/2004

Division of Nuclear Physics Meeting, Tuscon, AZ, "Measuring the Strange Spin of the Nucleon in MiniBooNE", 10/31/2003

Special Presentation to the National Science Foundation on MiniBooNE, Washington, DC, "Neutral Current Elastic Scattering in MiniBooNE", 10/28/2003

New Perspectives, Fermilab, Batavia, IL, Poster: "Proton Keep On Turnin'; Proud BooNE Keep On Burnin"”, 6/11/2002 
"Measurement of Muon Neutrino Quasi-Elastic Scattering on Carbon", A. AguilarArevalo et al. PRL 100, 032301 (2008)

"Search for Electron Neutrino Appearance at the $\Delta m^{2} \sim 1 \mathrm{eV}^{2}$ Scale", A. AguilarArevalo et al. PRL 98, 231801 (2007)

"A large-volume detector capable of charged-particle tracking", R. Tayloe et al. NIM A 562, 198 (2006)

MiniBooNE Technical Note \#104 "State of The Neutral Current Analysis", C. Cox, $10 / 16 / 03$

Analysis Memo: "Neutral Current Elastic Scattering Analysis for WIN 2003", C. Cox, 9/16/03

MiniBooNE Technical Note \#86 "An Open $\nu_{\mu}$ Neutral Current Elastic Box", C. Cox, 5/23/03

"A Guide to Cross Sections at MiniBooNE", D. C. Cox et al., March 2003

MiniBooNE Technical Note \#74 "Tests of The Scintillation Properties of Mineral Oil at IUCF", P. Ockerse et al., 2/5/03 (rev. 4/26/03)

MiniBooNE Technical Note \#70 "Slow Monitoring and Nitrogen Systems for MiniBooNE, Version II", C. Cox et al., 12/6/02 